FY 1992

Report on

Lawrence

Berkeley

Laboratory

\title{
Laboratory Directed \\ Research and \\ Development Program
}

Lawrence Berkeley Laboratory

University of California

Berkeley, California 94720

\section{MASTER}

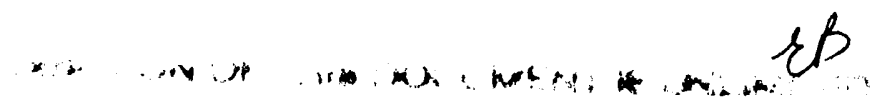




\section{Table of Contents}

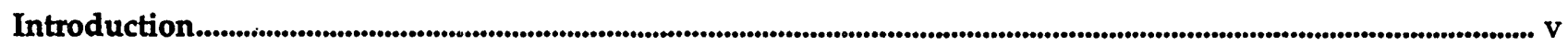

\section{Project Reports}

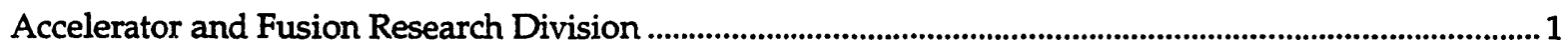

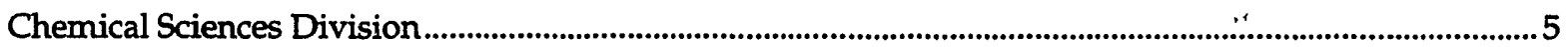

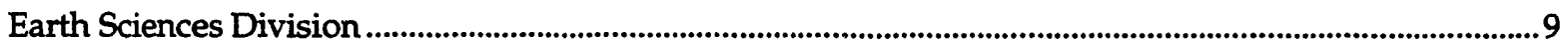

Energy and Environment Division ..............................................................................................................15

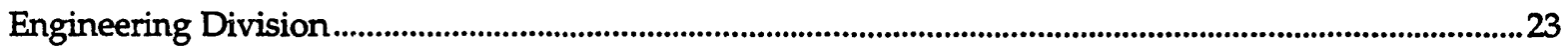

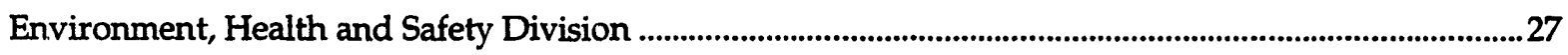

Information and Computing Sciences Division ................................................................................................... 29

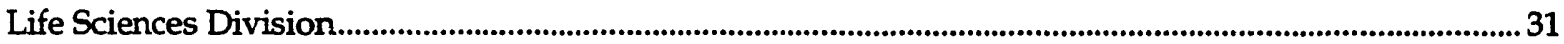

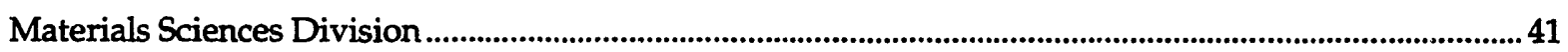

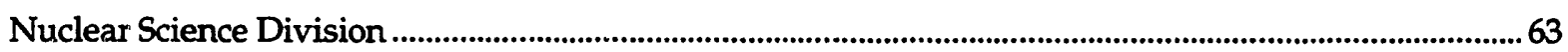

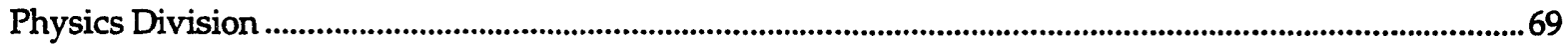

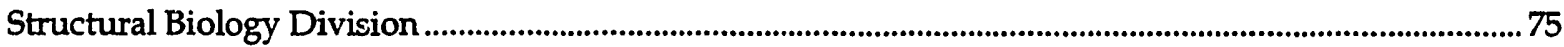

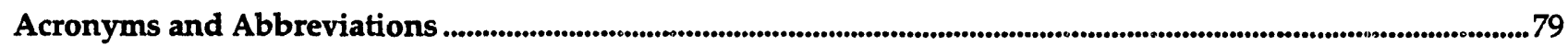




\section{Introduction}

The Lawrence Berkeley Laboratory (LBL) Laboratory Directed Research and Development Program FY 1992 report is compiled from annual reports submitted by principal investigators following the close of the fiscal year. This report describes the projects supported and summarizes their accomplishments. It constitutes a part of the Laboratory Directed Research and Development (LDRD) program planning and documentation process that includes an annual planning cycle, projection selection, implementation, and review.

The LBL LDRD program is a critical tool for directing the Laboratory's forefront scientific research capabilities toward vital, excellent, and emerging scientific challenges. The program provides the resources for LBL scientists to make rapid and significant contributions to critical national science and technology problems. The LDRD also advances the Laboratory's core scientific capability and permits exploration of exciting new opportunities. Areas eligible for support include:

- Work in forefront areas of science and technology that enrich Laboratory research and development capability;

- Advanced study of new hypotheses, new experiments, and innovative approacines to develop new concepts or knowledge;

- Experiments directed toward proof of principle for initial hypothesis testing or verification; and

- Conception and preliminary technical analysis to explore possible instrumentation, experimental facilities, or new devices.

The LDRD program supports LBL's mission in many ways. First, because LDRD funds can be allocated in a relatively short time frame, LBL researchers can support the mission of DOE and serve the needs of the nation by quickly responding to forefront scientific problems. Second, LDRD enables the Laboratory to attract and retain highly qualified scientists, and supports their efforts to carry out world-leading research. Finally, the LDRD program also supports new projects that involve graduate students and postdoctoral fellows, thus contributing to the education mission of the Laboratory.

LBL has a formal process for allocation of funds for LDRD. The process relies on individual scientific investigators and the scientific leadership of the Laboratory to identify opportunities that will contribute to scientific and institutional goals. The process is also designed to maintain compliance with DOE Orders, in particular DOE Order 5000.4A. From year to year, the distribution of funds among the scientific program areas will change. This flexibility optimizes the Laboratory's ability to respond to opportunities.

Laboratory LDRD policy and program decisions are the responsibility of the Laboratory Director. The Director has assigned general programmatic oversight responsibility to the Laboratory Deputy Director. Administration and reporting on the LDRD program is supported by the Office for Planning and Development. LDRD accounting procedures and financial management are consistent with the Laboratory's accounting principles and. stipulations under the contract between the University of California and the Department of Energy, with accounting maintained through the Laboratory's Chief Financial Officer.

In FY 1992, LBL was authorized by the Department of Energy to establish a funding ceiling for LDRD of 3\% of the Laboratory's FY 1992 operating budget. This funding level was provided to develop new scientific ideas and opportunities and allow the Laboratory Director an opportunity to initiate new directions. However, budget constraints limited available resources, so only $\$ 4.9 \mathrm{M}$ was allocated.

In FY 1992, scientists submitted 111 proposals requesting almost $\$ 12 \mathrm{M}$. A total of 48 projects were funded in FY 1992, with awards ranging from $\$ 11 \mathrm{~K}$ to $\$ 430 \mathrm{~K}$. These projects are summarized in Table 1 . 
Table 1. FY 1992 Laboratory Directed Research and Development Program.

Division Investigator

Project Title

Accelerator and Fusion Research Division

S. Chattopadhyay Generation of Ultrashort $x$-Ray Pulses and Ultraviolet Free

360,000 Electron Lasers

D. Dietderich

Phase Equilibria and Microstructural Texture in Bi-Oxide High- $\mathrm{T}_{\mathrm{c}}$ Superconductors

49,900

Chemical Sciences Division

R. Bergman Exploration of the Interface Between Homogeneous and Heterogeneous Catalysis

69,600

C. Harris

Ultrafast Dynamics of Electrons at Surfaces

125,400

W. Lester

Quantum Dynamics of Surface Reaction

24,000

D. Shirley/Z. Hussain VUV/Soft X-Ray Interferometry

58,800

Earth Sciences Division

J. Apps

Synthesizing Optical $\mathrm{PbSO}_{4}$ for Advanced Scintillation Applications

70,000

D. DePaolo

Determination of $\delta^{18} \mathrm{O}$ of Seawater in the Deep Ocean during the Last Glacial Maximum

26,500

T.V. McEvilly

Displacement Sensors for Seismology

48,000

Energy and Environment Division
S. Cramer
D. Grether
R. Ritschard
M. Rubin

X-Ray Absorption Spectroscopy

251,300

Simulation of an Advanced Utility-Customer Interface

75,000

New Approaches to Assessing Oceans as $\mathrm{CO}_{2}$ Sinks

129,300

Short-Wavelength Materials: $\mathrm{GaN}$ and $\mathrm{SiC}$

75,000

Engineering Division
S. Kleinfelder
Custom Gallium Arsenide Integrated Circuit Design for Applications in Nuclear and Particle Physics

J. Millaud/R. Ely

Development of a Capability for the High Density Packaging of

High Frequency Integrated Circuits

60,000

C. Rossington

Extended X-Ray Absorption Fine Structure Spectroscopy (EXAFS) in the $1-4 \mathrm{keV}$ Region

75,500

Environment, Health and Safety Division

R. Sun

Modification of an Andersson-Braun-Type Remmeter to Extend Its Sensitivity to $400 \mathrm{MeV}$

98,900 
Table 1. Continued.

\section{Information and Computing Science Division}

F. Olken

Life Sciences Division

J. Campisi

J. Chasis

T. Forte

R. Glaeser

T. Head-Gordon

M. Maestre

J. Rine

P. Yaswen

Robust Shotgun DNA Sequencing Assembly

82,900

Analysis of a Cell Cycle Regulatory Gene

100,100

Application of the Micropipette Technique to the Measurement of Erythroid Viscoelastic Properties during Terminal Differentiation

20,700

Environmental Air Pollutants and Oxidative Stress $\quad 90,500$

Baseline Data for Soft x-Ray Microscopy

40,000

A Comprehensive Approach to Protein Folding

10,800

Single DNA Molecule Chemistry and Manipulation for Gene Separation

87,400

Experimental Testing of Novel Mismatch Repair Enzymes for Mapping Natural Genetic Polymorphisms

100,000

Maintenance of Phenotypic Traits in Cultured Human Mammary Epithelial Cells

Materials Sciences Division

D. Attwood

Resist Modeling for X-Ray Lithography

E. Bourret

MOCVD Growth and Characterization of II-VI Compounds

Using Nontoxic Single-Molecule Precursors

D. Chemla

Optical Investigations of Quantum Well Structures under High Magnetic Fields

426,100

J. Clarke

High- $T_{c}$ Superconducting Magnetometer

56,300

C. Fadley

Novel Surface and Interface Studies Using Photoelectron Spectroscopy Combined with Scanning Tunneling Microscopy

129,000

60,000

A. Glaeser/R. Cannon Ceramic-Ceramic Joining via a Transient Liquid Phase

114,800

M. O'Keefe

Transport Spectroscopy of Quantum Dots

40,000

J. Orenstein

Preliminary Studies of Quantitative Electron Detectors

176,000

C. Shank

Time-Domain Spectroscopy of Semiconductors and Superconductors

123,700

M. Van Hove

Femtosecond Dynamics in Condensed Matter

137,400

W. Walukiewicz

Picometer-Scale Structure of Interfaces from synchrotron Radiation

50,000

E. Weber

Processing of Semiconductor Materials under High Hydrostatic

Pressure

104,300

Interfaces in Cross-Section

198,400 
Table 1. Continued.

Division Investigator Project Title

\section{Nuclear Science Division}

S. Freedman

Laser Trapping of Neutral Atoms

99,600

J. Harris

New Concepts for High Multiplicity Tracking Detectors

201,800

J.M. Nitschke

New Concepts in Isospin Studies

63,400

Physics Division

M. Levi

Data Acquisition Electronics

87,700

D. Nygren

Using Crude Time Walk Measurements to Improve Spatial Resolution

74,700

N. Roe

New Si Microstrip Disk Detector for Vertex Reconstruction of $B$

Meson Decays

178,000

Structural Biology Division

J. Hearst

X-Ray Microscopy and Microimaging of Eukaryotic Chromosomes

48,400

S. Holbrook

$X$-Ray Crystallographic Studies of RNA and DNA

68,100

TOTAL

$4,701,600$ 


\section{Accelerator Fusion and Research Division}

\section{Generation of Ultrashort X-Ray Pulses and Ultraviolet Free Electron Lasers}

Principal Investigators: Swapan Chattopadhyay and Kwang-Je Kim

Funding: $\begin{aligned} & \$ 360,000 \text { (FY92) } \\ & \$ 348,100 \text { (FY91) } \\ & \$ 332,200 \text { (FY90) }\end{aligned}$

\section{Project Description}

The purpose of this project was to put significant effort into a search for methods and techniques of generating ultrashort pulses (sub-picosecond) of electromagnetic radiation at short wavelengths ( $x$-rays, for example) and to continue our investigations into the physics and technology of free electron lasers (FELs) for applications at short wavelengths (ultraviolet and beyond). The program of research approached the above mission along the following broad fronts: (1) ultrashort pulse generation, (2) basic FEL research and study and, (3) necessary accelerator R\&D.

Ultrashort pulses of sub-picosecond duration in the $x$ ray wavelengths are expected to have a significant impact on condensed matter physics, chemistry, biochemistry and on studies of all ultrafast phenomena. Generation of short wavelength radiation via FELs will allow fundamental research at the cutting edge of material and life sciences. All these investigations are driven by a motivation to study advanced radiation facilities beyond the Advanced Light Source (ALS) at Berkeley.

\section{Accomplishments}

In the domain of basic FEL physics, the FEL spectral bandwidth, peak power, and effects of transverse oscillations in a fully three-dimensional FEL model have been poorly understood subjects so far, especially for applications to short wavelengths. We made significant progress in these areas at LBL in FY92 (K.-J. Kim, 1992; Y.H. Chin et al., 1993; Y.H. Chin et al., 1992; K.-J. Kim and M. Xie, 1992; C. Pellegrini et al., 1992). In particular, we have derived explicit expressions for the limiting spectral bandwidths in FEL oscillators, which indicates that they are sensitive to particular accelerator drivers due to different saturation processes. The result could be a decisive factor in optimizing accelerator systems for short wavelength FELs (C. Pellegrini et al., 1992). In the long wavelength infrared region, we have made significant progress in our understanding of hole-coupled optical resonators (W.P. Lumans et al., 1992; S. Krishnagopal et al., 1992) and in diagnostic techniques for single-pulse spectrum and width measurements for an infrared FEL (W.P. Lumans et al., 1992, LBL-32223). We have also studied the performance of Cerenkov FELs (J.-S. Choi et al., 1992) as a potential way to provide coherent radiation at wavelengths longer than $50 \mathrm{~mm}$, typical of conventional infrared FELs. In addition, we have initiated very basic studies of propagation and characterization of optical coherence through optical elements (K.-J. Kim and R.G. Littlejohn, in preparation).

We also investigated various approaches to short pulse production, e.g., a chirped FEL scheme, Compton backscattering of laser beams against chirped electron beams, scattering off a high-energy beam in a storage ring, etc. Out of our investigations, a very attractive and promising route to generate short pulse $x$-rays has been identified (S. Chattopadhyay and K.-J. Kim, 1992). This technique involves Compton scattering a short laser pulse in the visible light ranges (few tens to few hundreds of femtoseconds @ $0.5 \mathrm{~mm}$ say) off a low-energy electron beam of normal duration ( 10 ps) at right angles to each other. The short pulse radiation is produced in the direction of the electron beam. Simple estimation shows (S. Chattopadhyay and K.-J. Kim, 1992; K.-J. Kim et al., in preparation) that a 25$\mathrm{MeV}$ electron beam containing $1.6 \mathrm{nCoulomb}\left(10^{10}\right.$ electrons) of charge per $10 \mathrm{ps}$ bunch with a normalized emittance of $5 \mathrm{~mm}$-mrad, when focused down to 100 $\mathrm{mm}(\sim 330 \mathrm{fs})$ could completely overlap transversely a $0.5 \mathrm{~mm}, 330 \mathrm{fs}$ laser beam and scatter off to produce $1-\AA$ x-ray bursts of 330 fs duration containing $2.7 \times 10^{5}$ photons, if the laser intensity is of the order of $3 \times 10^{19}$ $\mathrm{W} / \mathrm{m}^{2}$.

Such electron beams can be produced by a small room temperature linac and a suitable electron gun ( $\mathrm{rf}$ photocathode microwave gun or a gun-buncher system similar to that employed in the ALS). The low emittances required have already been achieved in guns at LANL, BNL, UCLA, etc. The focusing of the electron beam is tight, with the necessary b-values of 
$2.4 \mathrm{~cm}$, but is achievable. The femtosecond laser with necessary power-density can be developed in a straightforward manner. This was a major advance in terms of conception and preliminary estimations. The next step should be toward a conception of short pulse $x$-ray detection and a proof-of-principle demonstration.

\section{Publications}

S. Chattopadhyay and K.-J. Kim, "Generation of Subpicosecond X-rays," LBL-31968 (1992).

Y.H. Chin, K.-J. Kim, and M. Xie, "Calculation of 3-D Free Electron Laser Gain: Comparison with Simulation and Generalization to Elliptical Cross Section," submitted to the 14th International FEL Conference, Kobe, Japan, Aug. 23-28, 1992, LBL-32287.

Y. H. Chin, K.-J. Kim, and M. Xie, "Three-Dimensional FEL Theory Including Betatron Oscillation," to be published in Phys. Rev. A. (1993).

J.-S. Choi, K.-J. Kim, and M. Xie, "Pulse Propagation in Cerenkov Free Electron Laser," submitted to the 14th International FEL Conference, Kobe, Japan, Aug. 2328, 1992.

K.-J. Kim, "Calculation of Peak Power for Short Wavelength FELs," presented at the Workshop on 4th Generation Light Sources, Stanford, CA, Feb. 19, 1992, LBL-31969.

K.-J. Kim, S. Chattopadhyay, and C.V. Shank, "Generation of Sub-Picosecond X-rays by $90^{\circ}$ Compton Scattering," in preparation.

K.-J. Kim and R.G. Littlejohn, "Coherence Criteria in Terms of Entropy and Phase Space Area," in preparation.

K.-J. Kim and M. Xie, "Self-Amplified Spontaneous Emission for Short Wavlength Coherent Radiation," submitted to the 14th International FEL Conference, Kobe, Japan, Aug. 23-28, 1992, LBL-32288.

S. Krishnagopal, M. Xie, and K.-J. Kim, "A Mechanism for Suppression of Mode Beating in a Saturated Hole Coupled FEL Oscillator," submitted to the 14th International FEL Conference, Kobe, Japan, Aug. 2328, 1992, LBL-32223.

W.P. Leemans, J.A. Edighoffer, K.-J. Kim, S. Chattopadhyay, and H.A. Schwettman, "A Technique for Single-Pulse Spectrum and Pulse Width Measurements for an IR-FEL," submitted to the 14th International FEL Conference, Kobe, Japan, Aug. 23-28, 1992, LBL-32284.
W.P. Leemans, M. Xie, J.A. Edighoffer, E. Wallace, K.-J. $\mathrm{Kim}$, and S. Chattopadhyay, Experiment and Simulation of Hole-coupled Resonator Modes," submitted to the 14th International FEL Conference, Kobe, Japan, Aug. 23-28, 1992, LBL-32285.

C. Pellegrini, J. Rosenzweig, H.-D. Nuhn, P. Pianeta, R. Tatchyn, H. Winick, K. Bane, P. Morton, T. Raubenheimer, J. Seeman, K. Halbach, K.-J. Kim, and J. Kirz, "A 2 to 4 nm High Power FEL on the SLAC Linac," submitted to the 14th International FEL Conference, Kobe, Japan, Aug. 23-28, 1992.

\section{Phase Equilibria and Microstructural Texture in Bi-Oxide High- $T_{C}$ Supercon- ductors}

Principal Investigators: Daniel R. Dietderich and Ronald M. Scanlon

Funding: $\$ 49,900$

\section{Project Description}

This program would continue the research on bi-oxide superconductors that began in Japan. That work showed that composition and processing modifications can promote the alignment of $\mathrm{Bi}_{2} \mathrm{Sr}_{2} \mathrm{CaCu}_{2} \mathrm{O}_{8}$ grains in thick films responsible for high-critical current densities. However, most of that work was empirical and aimed at fabricating conductors with higher-critical currents. It did not address the fundamental factors affecting grain alignment. In contrast, the work proposed here is intended to provide understanding of the mechanistic aspects of grain alignment in a more fundamental manner.

To accomplish this, a doctor-blade processed tape has been studied to determine a grain alignment mechanism in the $\mathrm{Bi}_{2} \mathrm{Sr}_{2} \mathrm{CaCu}_{2} \mathrm{O}_{8}$ compound during melt processing. This tape, due to its sample geometry with a free surface, permits easy in situ and post heattreatment characterization of samples. We are performing post heat-treatment $x$-ray diffraction, SEM (scanning electron microscopy) with EDS (energy dispersive spectrometry), and EBSP (electron back scattering patterns), and TEM (transmission electron microscopy) analysis of these samples to identify a grain alignment mechanism during melt processing.

In the near future an alternative dip-coating process will also be developed at LBL. This process has the potential to produce long lengths of conductor re- 


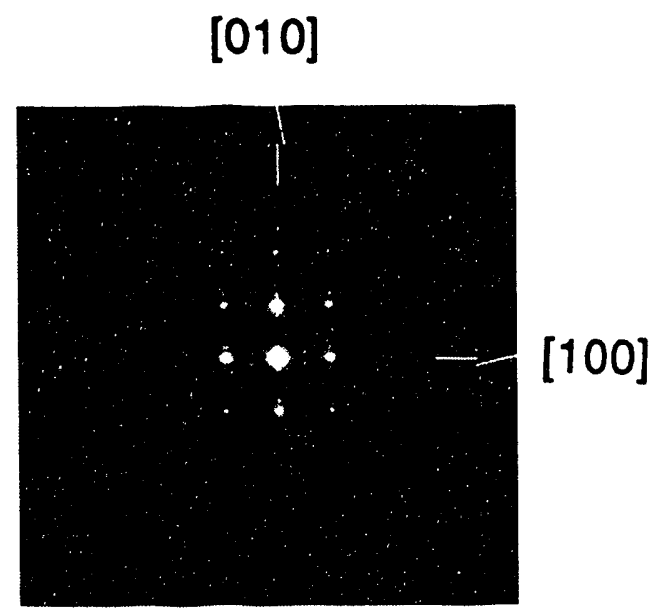

(a)

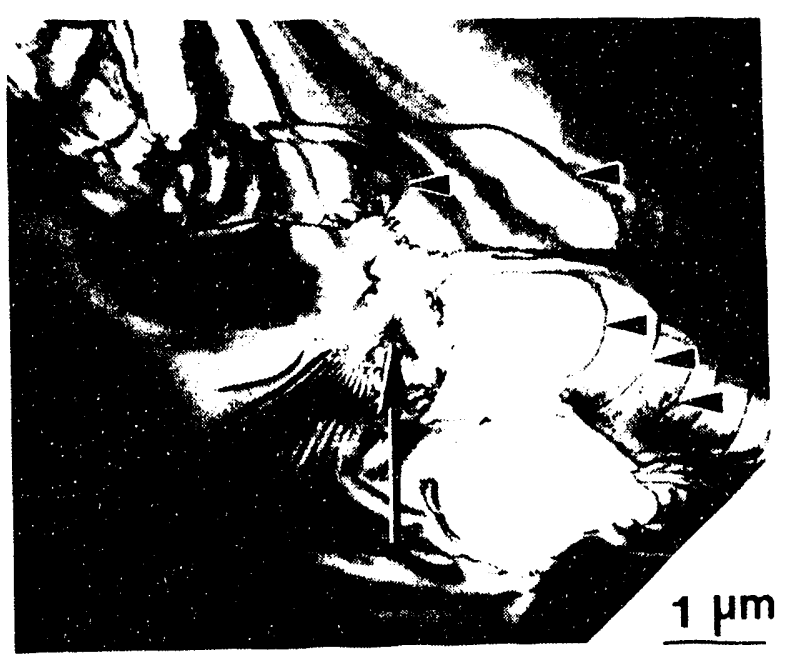

(b)

Fig. 1. Transmission electron microscope (a) 001 diffraction pattern and (b) image for a $\mathrm{Bi}_{2} \mathrm{Sr}_{2} \mathrm{CaCu}_{2} \mathrm{O}_{x}$ doctor-blade tape melt processed by heating to $890^{\circ} \mathrm{C}$, slow cooling to $870^{\circ} \mathrm{C}$, and then quenching to room temperature.

quired for various magnet applications as well as providing high current density grain-aligned homogenous material for the study of microstructural texturing and phase formation.

\section{Accomplishments}

This work is the first to observe a possible grain alignment mechanism for the $\mathrm{Bi}_{2} \mathrm{Sr}_{2} \mathrm{CaCu}_{2} \mathrm{O}_{y}$ phase. Our microstructural studies suggest that textured $\mathrm{Bi}_{2} \mathrm{Sr}_{2} \mathrm{CaCu}_{2} \mathrm{O}_{\mathrm{y}}$ forms on cooling by a nucleation and growth process. TEM observations show that the $\mathrm{Bi}_{2} \mathrm{Sr}_{2} \mathrm{CaCu}_{2} \mathrm{O}_{4}$ phase may nucleate on particles present in the high-temperature melt and grow by a ledge-type mechanism. For example, the arrow in the center of Figure 1(b) denotes the particle while the small arrow heads denote the ledges that we believe have grown from it. These observation also suggest that the fast growth direction is [100] (the diffraction pattern in Figure. 1(a)). Future work will be directed at confirmation of this mechanism, for if this mechanism is correct controlling the distribution and orientation of the particles present in the liquid oxide at high temperature should produce a material with better grain alignment and, as a result, a higher-critical current.

\section{Publications}

D.R. Dietderich, "Textured Thick Films of $\mathrm{Bi}_{2} \mathrm{Sr}_{2} \mathrm{CaCu}_{2} \mathrm{O}_{x}$," presented at the Spring Meeting of the Materials Research Society, San Francisco CA, April-May 1, 1992.

D.R. Dietderich, "Noble Metals and $\mathrm{Bi}_{2} \mathrm{Sr}_{2} \mathrm{CaCu}_{2} \mathrm{O}_{x}$ " presented at the Applied Superconductivity Conference, Chicago, $\Pi$, August 23-28, 1992. 


\section{Chemical Sciences Division}

\section{Exploration of the Interface Between Homogeneous and Heterogeneous Catalysis: Synthesis and Study of Organometallic Catalysts Supported on Novel Polysiloxane Materials with Controllable Solubility Properties}

Principal Investigators: Robert G. Bergman and Bruce M. Novak

Funding: $\$ 69,600$

\section{Project Description}

Attaching transition metal catalysts to silica supports has become a general method for dispersing the catalyst particles, thereby achieving high activity in catalytic reactions. Because of the insoluble nature of these supported catalysts, it has been difficult to obtain detailed information about their structures and reaction mechanisms. The goal of this project is the use of "arrested" hydrolysis of hydrated sodium metasilicate and tetraalkoxysilanes to generate silica fragments small enough to be soluble in conventional organic solvents, and then to bind metal catalysts to these supports. The solubilized supported catalysts prepared in this way should be amenable to investigation by powerful techniques such as solution phase nuclear magnetic resonance (NMR) and other spectroscopic methods, which should provide detailed structural and mechanistic information on these materials.

\section{Accomplishments}

Poly(silicic acid) has been prepared by the acidcatalyzed hydrolysis of hydrated sodium metasilicate in aqueous solution. Extracted into THF, the polymer can be esterified easily with benzyl alcohol $\left(\mathrm{C}_{6} \mathrm{H}_{5} \mathrm{CH}_{2} \mathrm{OH}\right)$ by azeotropic distillation under atmospheric pressure. Soluble silylated silicic acid ester polymers of molecular weight near 2,000 and polydispersity index near 1.5 have been obtained in this way. As hoped, these polymers give clear ${ }^{1} \mathrm{H}$ and ${ }^{29} \mathrm{Si} \mathrm{NMR}$ spectra. The spectra allow us to determine to good accuracy the degree of incorporation of benzyl
$\left(\mathrm{C}_{6} \mathrm{H}_{5} \mathrm{CH}_{2}\right)$ groups. This extent of incorporation, as well as the molecular weight and percentage of branching of the benzylated silicic acid esters, can be controlled by varying the reaction time and conditions. Subsequent silylation of the esterification products derivatizes the remaining silica-bound hydroxyl groups, and this permits the convenient isolation of polysiloxanes that are inert to further self-condensation.

The next stage of the project provided a water-free method for removing benzyl groups from the polymer. Treatment of the benzylated polysiloxane with gaseous hydrogen in the presence of a metal catalyst successfully achieves this goal. The best conditions for regenerating polymer-attached $\mathrm{Si}-\mathrm{OH}$ groups utilizes $10 \% \mathrm{Pd}$ on activated carbon as catalyst in ethyl acetate or methyl acetate solvent under $3 \mathrm{~atm}$ of $\mathrm{H}_{2}$ at room temperature for several hours. Present efforts are directed at utilizing the silanol groups as active sites for binding homogeneous transition metal catalysts to these soluble supports. Catalyst precursors such as $\left[\mathrm{Rh}\left(\mathrm{CH}_{2} \mathrm{CH}_{2} \mathrm{CH}_{3}\right)_{3}\right]_{2}$ and tetrakis(neopentyl) zirconium are currently under investigation.

\section{Ultrafast Dynamics of Electrons at Surfaces}

Investigator: Charles Harris

Funding: $\$ 125,400$

\section{Project Description}

We are developing techniques to probe the spectroscopy and dynamics of surface electronic excitations with unprecedented precision and time resolution. Using a powerful new instrumental approach to twophoton photoemission, we have begun a wide-ranging exploration of the interactions of excited electrons with atomically thin dielectric films on metal surfaces. We will use fiber optic pulse compression techniques with a synchronously pumped dye laser to do time- and angle-resolved two-photon photoemission. This would enable the direct measurement of lifetimes and effective masses of excited electrons on the 
femtosecond time scale. This system will provide a unique ability to explore excited-state dynamics of . electrons at surfaces and interfaces with ultrafast time resolution.

\section{Accomplishments}

The completed two-photon photoemission spectrometer, combining a 2-MHz repetition-rate laser with time-of-flight electron-energy analysis, has demonstrated unparalleled energy resolution and sensitivity. The new angle-resolved manipulator with liquidhelium cooling has enabled measurements of band dispersion and effective masses on a variety of systems. The laser pulse-compression system has been constructed and is undergoing tests before being integrated into the photoemission system. Ultrashort light pulses of 150 femtosecond length have been generated in both the visible and ultraviolet. The wavelength range of the dye laser system has been extended.

We have examined rare gas monolayers, multilayers of alkanes, and chemisorbed thiols and sulfur on the silver (111) surface. The layer-by-layer growth of alkane films on $\mathrm{Ag}(111)$ and the evolution to bulk dielectric properties have been observed. Spectra of cyclohexane for increasing coverage are shown in Figure 1. We have discovered that electrons bound to

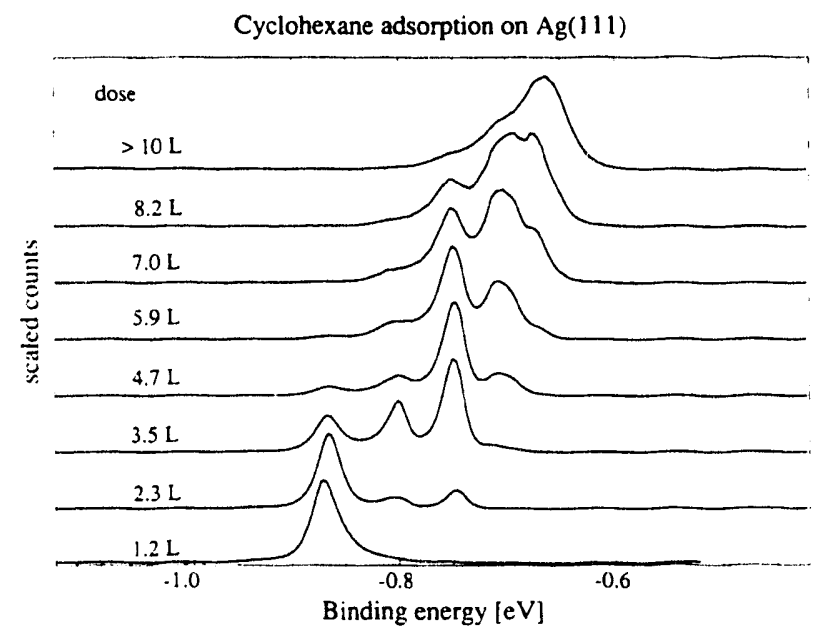

Fig. 1. See text. monolayer films of $\mathrm{Xe}$ on $\mathrm{Ag}(111)$ have a lower effective mass than do electrons bound to the bare metal. On the monolayer covered surface the electron's effective mass becomes identical to that of a 2-D free electron. This implies that a single atom thick layer decouples the electron from the band structure of the metal.

Evidence of electron localization has been found in two molecule thick adsorbed films. Bilayers on hexane, heptane, and octane show a new non-dispersive feature in angle-resolved two-photon photoemission spectra, as seen in Figure 2. This is the first observation of its type and demonstrates that the new technique and apparatus developed in this project is ideally suited to observations of electron dynamics and localization in thin films.

$\mathrm{N}=1$ Dispersion of a Bilayer of $\mathrm{n}-\mathrm{Heptane}$ on $\mathrm{Ag}(111)$

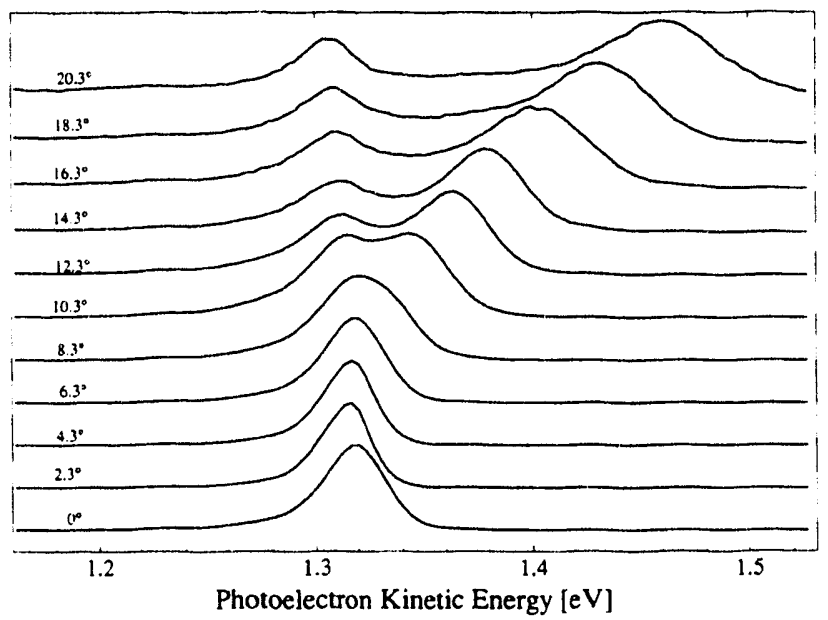

Fig. 2. See text.

\section{Publications}

D.F. Padowitz, W.R. Merry, R.E. Jordan, and C.B. Harris, "Two-Photon Photoemission as a Probe of Electron Interactions with Atomically Thin Dielectric Films on Metal Surfaces," Phys. Rev. Lett., in press.

W.R. Merry, R.E. Jordan, D.F. Padowitz, and C.B. Harris, "Image-Potential States in the Presence of Thin Adsorbed Films," in preparation. 


\section{Quantum Dynamics of Surface Reactions}

Principal Investigators: William A. Lester, Jr. and Vladimir Z. Kresin

Funding: $\$ 24,000$

\section{Project Description}

The research is concerned with the quantum theory of surface chemical reactions. The major goal is to develop a state-to-state description of surface reactions and to evaluate the product energy distribution in the presence of the surface that is a strong catalytic agent. The reaction Hamiltonian method, developed in our group, will be applied to study the interaction between the surface and adsorbed reactants.

\section{Accomplishments}

A quantum-mechanical model for heterogeneous catalytic reaction has been developed. It is shown that the presence of the surface leads to additional channels of reaction. The interaction between reactants and surface leads to an increase in reaction rate owing to an increase in a Franck-Condon factor. We studied atomic as well as molecular adsorption. The additional channels are found to dominate the exponential smallness of the reaction probability of the direct channel (i.e., in the absence of the surface) producing large reaction probabilities for surface-catalyzed reaction. The model has been applied to selected reactions including hydrogenation of ethylene.

\section{Publication}

V.Z. Kresin and W.A. Lester, Jr. "Quantum-Mechanical Model of Heterogeneous Catalysis," Chem. Phys. Lett. 197, 1 (1992).
VUVISoft X-Raÿ Interferometry: A Proposal for Producing Ultrahigh Resolution Spectra from Broad-Band - Synchrotron Radiation

Principal Investigators: David A. Shirley, Malcolm R. Howells, Zahid Hussain, and Tobias Reich

Funding: $\$ 58,800$

\section{Project Description}

High-energy photon sources have historically had very low intensities, and the traditional intensi'tyversus-resolution balance has been strongly tilted in favor of the highest achievable intensity at some very modest resolution, typically between 100 and 1000 for photon energies between $30 \mathrm{eV}$ (the upper limit of normal-incidence optics) and $1000 \mathrm{eV}$. In fact, the world's record in this range is ca 10,000. The goal of this project was to achieve high resolution in the $\mathrm{VUV} /$ soft $\mathrm{x}$-ray energy range $(20-100 \mathrm{eV})$ using two independent approaches:

1. To make the necessary improvements in the 6-m Toroidal Grating Monochromator (TGM) at Stanford Synchrotron Radiation Laboratory (SSRL) to achieve theoretically predicted slit-limited resolution of 30,000.

2. To propose a novel scheme for analyzing broadband high-energy photon beams with unprecedentedly high resolution, using grazing-incidence interferometry. The proposed device, based on existing state-of-the-art technology, should provide the capability of stepping the pathlength differences with precision and accuracy high enough to resolve spectral features in a VUV/soft $x$-ray beam to 1 part in $10^{6}$ or better.

High-resolution study of gases provides information about electron-electron correlation for which helium is used as a prototype neutral system. The discovery of 
autoionizing states of helium by Madden and Codling led to the discovery of new correlation quantum number. Recent results from photoionization studies of helium have yielded a wealth of new information about the double excitation states of helium, and strongly supports the need of high-resolution capabilities.

\section{Accomplishments}

This project has accomplished the first goal by achieving the world best resolving power of 14,000 at $65 \mathrm{eV}$. Figure 3 shows the photoionization spectrum from helium near the double excitation states obtained

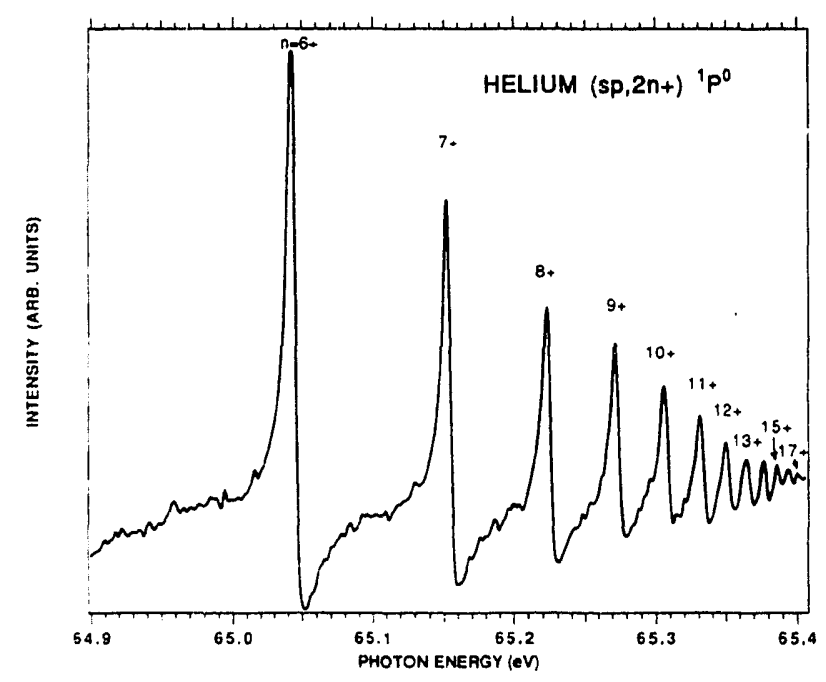

Fig. 3. Autoionizing states of double-excited He below the $N=2$ threshold of $\mathrm{He}^{+}$, showing only the $n \geq 6$ region. The spectrum was taken using entrance and exit slits of $15 \mu \mathrm{m}$, with a resolving power of $E / \Delta E=13,000$. using the modified TGM at SSRL. With high resolution it was possible to resolve up to 17 Rydberg states. The high resolution was obtained by considerably modifying the TGM at SSRL. This included replacing the entrance and exit slits with a pair of high precision slits and the encoder/stepping motor with highresolution units.

As far as the grazing-incidence interferometer is concerned, we have carried out computer-simulated design analysis. Initial results have indicated that a Mach-Zender interferometer with wavefront division of the beam is the most appropriate scheme for achieving this goal. We have successfully tested the highresolution encoder required for indexing the path length differences. The design of other components are under progress, and we have a great hope in the successful completion of this project.

We believe that by achieving resolution levels with high-energy (vacuum ultraviolet (VUV)/soft x-ray) radiation comparable to those available with lowenergy (visible/infrared) laboratory sources, the completion of this project would go a long way toward the intellectual unification and integration of synchrotron-radiation science with laboratory-based activities.

\section{Publication}

T. Reich, Z. Hussain, E. Moler, G. Kaindl, D.A. Shirley, and M. Howells, "High Resolution in the Soft X-ray Range with a Toroidal Grating Monochromator," presented at the Tenth International Conference on Vacuum Ultraviolet Radiation Physics, Paris, July 2731,1992 and to be submitted for publication in the Nuclear Instruments and Methods. 


\section{Earth Sciences Division}

\section{Synthesizing Optical $\mathrm{PbSO}_{4}$ for Advanced Scintillation Applications}

Principal Investigator: John A. Apps

Funding: $\$ 70,000$

\section{Project Description}

Preliminary screening studies by LBL scientists $S$. Derenzo and V. Moses, to identify materials with improved scintillation properties, led to the discovery of $\mathrm{PbCO}_{3}$ (cerussite) and $\mathrm{PbCO}_{4}$ (anglesite) as promising candidates. More recent characterization studies confirmed the scintillation properties of these two minerals and showed that those of anglesite are superior. The short fluorescence lifetime, high-density $\left(6.2 \mathrm{~g} . \mathrm{cm}^{-3}\right)$, and light output of the latter make it especially suitable for applications where high counting rates, good stopping power and nanosecond timing are important, e.g., medical imaging and nuclear science. Both minerals are resistant to damage from intense radiation and are chemically stable under ambient conditions. Neither will decompose nor require special precautions to protect them from the atmosphere.

Anglesite crystals of the necessary size and optical quality for scintillation applications do not occur naturally and must be synthesized if their exceptional properties are to have practical application. Several companies have expressed an interest in producing and marketing lead sulfate scintillators, though further research and development in methods of growing optically clear crystals is required.

A preliminary review of the literature indicated that the most practical method of growing optically clear anglesite crystals would be by the hydrothermal method. Hence the purpose of the project was to establish the feasibility of this technique through both theoretical and experimental studies.

\section{Accomplishments}

The stability field of anglesite in relation to other lead salts in the system $\mathrm{PbO}-\mathrm{SO}_{3}-\mathrm{HCl}-\mathrm{NaCl}-\mathrm{H}_{2} \mathrm{O}$ was defined between $25^{\circ} \mathrm{C}$ and $0.1 \mathrm{MPa}$, and $700^{\circ} \mathrm{C}$ and 100 $\mathrm{MPa}$. Thermodynamic data for all known solid phases in the referenced system and all known species in the aqueous phase were compiled and evaluated usin : the Helgeson-Kirkham-Flowers equation of state. The solubility of anglesite in $\mathrm{NaCl}$ solutions over the same range of temperatures and pressures was calculated.

Experimental studies to confirm theoretical predictions of anglesite solubility were conducted at $25,95,150$, $175,200,225,250$, and $275^{\circ} \mathrm{C}$, in $10^{-3} \mathrm{~m}$ or $3.2 \mathrm{~m} \mathrm{NaCl}$ solutions. Although the experimental results, theoretical predictions, and limited solubility data in the literature are still being interpreted, preliminary findings indicate that the traditional thermal gradient method for growing single crystals can be accomplished only in solutions of at least $1 \mathrm{~m}$ aqueous chloride in order to obtain an acceptable temperature coefficient of solubility. The solutions must also be mildly acid with $\mathrm{pH}<5.0$ in order to prevent coprecipitation of lanarkite $\left(\mathrm{PbSO}_{4} \cdot \mathrm{PbO}\right)$.

Experimental studies were conducted at $95^{\circ} \mathrm{C}$ to nucleate and grow seed crystals after a method recommended in the literature. Seed crystals were successfully grown by this method. Several attempts were made to grow crystals at $75-90^{\circ} \mathrm{C}$ and various $\mathrm{NaCl}$ concentrations using glass apparatus. In all cases, the experiments had to be terminated for various technical reasons. Crystal growth from seeds was successful, however, at $150^{\circ} \mathrm{C}$ in $2 \mathrm{~m} \mathrm{NaCl}(\mathrm{aq})$ in titanium autoclaves, although growth rates were too slow for practical purposes. A review of rates of crystal growth from experiments conducted during the course of this investigation, and information in the literature, indicates that optimum conditions for crystal growth are in slightly acid strong chloride solutions at temperatures $>350^{\circ} \mathrm{C}$. A 1 liter Hastelloy C-276 autoclave is being modified to conduct further crystal growth experiments at $400^{\circ} \mathrm{C}$ and $50 \mathrm{MPa}$. 


\section{Determination of $\delta^{18} \mathrm{O}$ of Seawater in the Deep Ocean during the Last Glacial Maximum}

Principal Investigators: Daniel P. Schrag and Donald J. DePaolo

Funding: $\$ 26,500$

\section{Project Description}

The $\delta^{18} \mathrm{O}$ values of foraminifera tests in deep sea sediments record episodes of glaciation over at least the 35 million years of Earth history. The $\delta^{18} \mathrm{O}$ of foraminiferal carbonate $\left(\delta^{18} \mathrm{O}_{f \mathrm{f}}\right)$ reflects a combination of the temperature and the $\delta^{18} \mathrm{O}$ of seawater $\left(\delta^{18} \mathrm{O}_{\mathrm{sw}}\right)$; the latter effect is a result of changes in the volume of low- $\delta{ }^{18} \mathrm{O}$ continental ice sheets. To understand global climatic changes between glacial and interglacial periods, it is necessary to determine how much of the measured change in foraminiferal $\delta^{18} \mathrm{O}$ from glacial to interglacial $\left(\Delta \delta^{18} \mathrm{O}_{\mathrm{fc}}\right)$ is produced by changing seawater temperature and how much by changing $\delta^{18} \mathrm{O}_{\text {sw. }}$. The purpose of this study is to develop a method for determining $\delta^{18} \mathrm{O}_{s w}$ in the deep ocean during the last glacial maximum, using isotopic analyses of pore fluids combined with modeling of the diffusive transport of oxygen through the sediment column. Because oceanic deep water is isotopically less variable than surface water, this method eliminates the uncertainties associated with previous determinations that measured surface water $\delta^{18} \mathrm{O}$ variations.

Our method is based on the observation that over the relatively short time interval since the Last Glacial Maximum (LGM), most sediments on the ocean floor behave as porous media with chemical transport in the pore fluids occurring primarily by diffusion. Over this time interval, the sediments can be considered essentially nonreactive with respect to oxygen because of the slow rate of diagenetic reactions and the fact that oxygen is a major component of both fluids and solids. The changes in $\delta^{18} \mathrm{O}_{\text {sw }}$ caused by glaciation and deglaciation represent a periodic boundary condition for the sediment-pore fluid system. These changes diffuse down from the sea floor, leaving a profile of $\delta^{18} \mathrm{O}$ versus depth in the pore fluid that is a record (albeit degraded) of the $\delta^{18} \mathrm{O}$ history of the overlying seawater. If there is no advection, the relationship between the $\delta^{18} \mathrm{O}$-de $\mathrm{Dth}$ record in pore fluid and the $\delta^{18} \mathrm{O}$-time history of t 'e ocean is determined only by the rate of self-diffusiol. of water in the sediment. For the sediment-pore fluid s:stem, the attenuation factor is such that modern pore fluid preserves little of the $\delta^{18} \mathrm{O}$ history of the ocean at the frequency of glacial cycles except for the large $\delta^{18} \mathrm{O}$ shift associated with the last deglaciation, which should appear as a high$\delta^{18} \mathrm{O}$ peak in the pore fluid $\delta^{18} \mathrm{O}$-depth profile at a depth of 25 to 50 meters (depending on the diffusivity). Thus, if the depth and amplitude of the pulse of higher $\delta^{18} O$ pore fluid can be measured accurately at a specific location, both the diffusivity of water in the sediments and the $\delta^{18} \mathrm{O}_{\mathrm{sw}}$ during the LGM can be determined. This approach is analogous to that used to calculate the thermal diffusivity of soils from temperature variations at the Earth's surface.

The pulse of high- $\delta^{18} \mathrm{O}$ pore fluid from the LGM should exist everywhere on the ocean floor where diffusion is the dominant transport mechanism, regardless of lithology. A survey of data from thirtyseven Deep Sea Drilling Project (DSDP) sites identifies several sites with positive deviations of $\delta^{18} \mathrm{O}$ relative to the normal decrease with depth. Sampling intervals on these and most other DSDF and Ocean Drilling Program (ODP) cores are too large to measure accurately the amplitude of the signal. One exception is Site 576 from DSDP Leg 86 in the western Pacific Ocean where seven samples were taken in the top 60 meters of core. Our approach in this study has been to use a model of oxygen isotope transport in pore fluids with parameters appropriate to Site 576 to obtain values of $\delta^{18} \mathrm{O}_{s w}$ that reproduce the measured pore fluid values. When higher resolution measurements are available from future ODP drilling legs (which will require closely spaced pore fluid samples to a depth of ca. $100 \mathrm{~m}$ ), a more precise determination of $\Delta \delta^{18} \mathrm{O}_{\mathrm{sw}}$ should be possible.

\section{Accomplishments}

The oxygen isotope measurements of pore fluids from Site 576 define a positive anomaly in $\delta^{18} \mathrm{O}$ with depth with the peak occurring between 20 and 35 meters below seafloor. Assuming that diffusion dominates chemical transport in the pore fluid $\left(\mathrm{U}_{f}=0\right)$, we obtain a best fit to the data when $\mathrm{k}=0.55\left(\mathrm{D}_{\text {eff }}=3.6 \times 10^{-6}\right.$ $\mathrm{cm}^{2} \mathrm{sec}^{-1}$ at the top of the sediment) and $\Delta \delta^{18} \mathrm{O}_{\mathrm{sw}}=$ $1.0 \%$ (Figure 1 ). Based on the results of calculations using other values of $\Delta \delta^{18} \mathrm{O}_{s w}$ (Figure 1), we estimate the error on this calculation to be $\pm 0.25 \%$. This error could be reduced with higher resolution sampling as the present fit is strongly dependent on the single measurement of seawater at the top of the sediment. A value of $1.0 \pm 0.25 \%$ is consistent with that obtained by Shemesh et al. for surface $\Delta \delta^{18} \mathrm{O}_{s w}$ but is lower than the value of $1.3 \%$ from Fairbanks. This difference is significant because if the average $\delta^{18} \mathrm{O}_{\mathrm{sw}}$ during the 


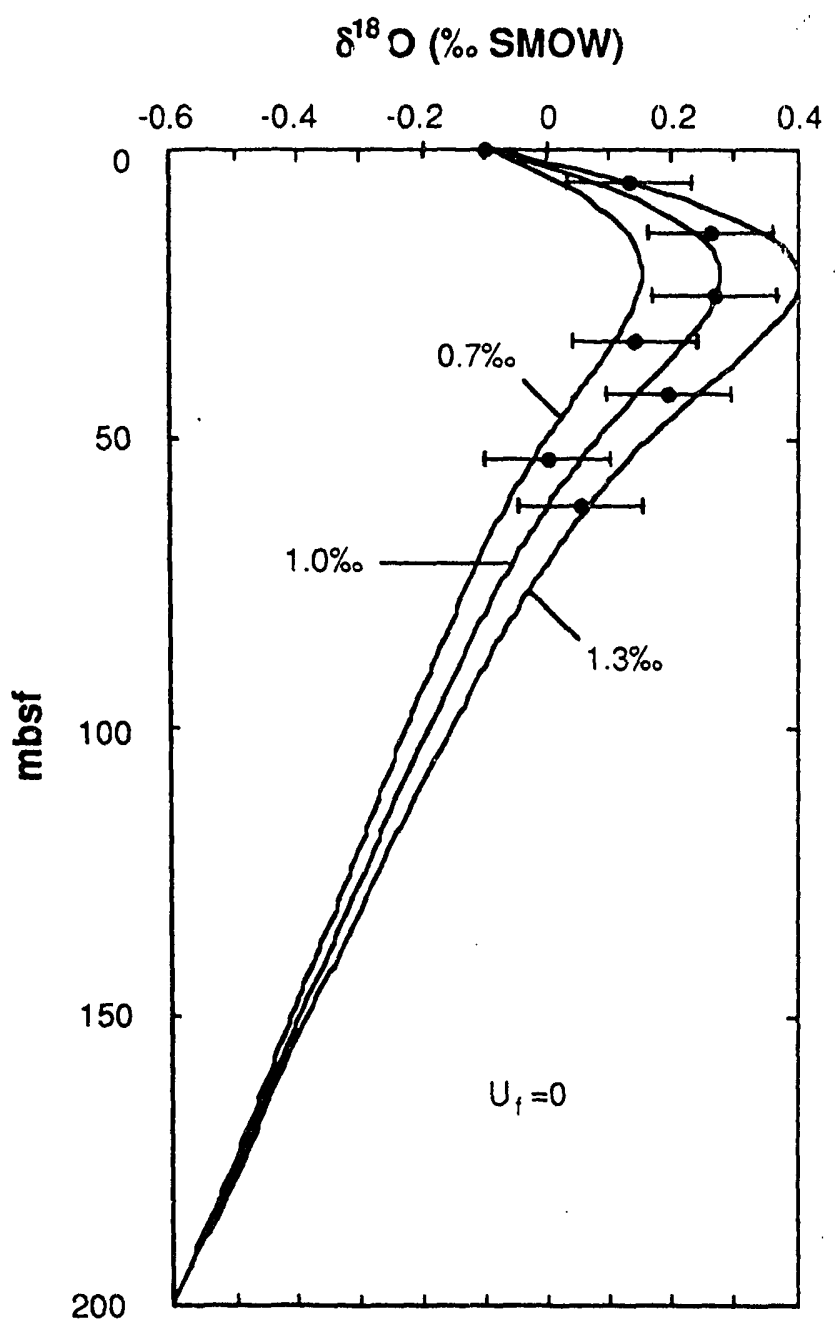

Fig. 1. Model results (lines) and pore fluid data (dots) for DSDP Site 576. The model lines are labeled with the value used for $\Delta \delta^{18} \mathrm{O}_{s w}$ the change in $\delta^{18} \mathrm{O}$ of seawater from the last glacial maximum to the present. In this model, there is no advection $\left(U_{f}=0\right)$. A best fit to the data is obtained for $k$ $=0.55\left(D_{\text {eff }}=3.6 \times 10^{-6} \mathrm{~cm}^{2} \mathrm{sec}^{-1}\right.$ at the top of the sediment $)$ and $\Delta \delta^{18} \mathrm{O}_{s w}=1.0 \%$. The error on this calculation, which we estimate to be $\pm 0.25 \%$, is illustrated by the lines generated for $\Delta \delta^{28} \mathrm{O}_{s w}=0.7$ and $1.3 \%$.

the LGM were $1.0 \%$ higher than today instead of $1.3 \%$, it would require the $\delta^{18} \mathrm{O}$ value of average LGM ice to be $-32 \%$ instead ci $-42 \%$. This change would imply a higher average temperature at the sites of ice accumulation, reducing estimates of glacial-to-interglacial temperature change in high latitude continental interiors and facilitating moisture transport to the sites of ice accumulation. A change in the accepted value of LGM $\delta^{18} \mathrm{O}_{\text {sw }}$ would also alter the LGM sea surface temperatures and salinities calculated from the $\delta^{18} \mathrm{O}$ values of planktonic foraminifera.
Our work on this project continued with the recent collection of pore fluids from Ocean Drilling Program Leg 145. By measuring the oxygen isotopic composition of pore fluids from Leg 145 using the vacuum line that was designed for this study, we hope to improve on the determination of $\delta^{18} \mathrm{O}_{\mathrm{sw}}$ during the LGM described above.

\section{Publications}

D. P. Schrag and D. J. DePaolo, "Determination of $\delta^{18} \mathrm{O}$ of Seawater in the Deep Ocean during the Last Glacial Maximum," Paleoceanography, in press.

\section{Displacement Sensors for Seismology}

Principal Investigators: Thomas V. McEvilly, Joseph E. Katz, William F. Kolbe, and Martin J. Pollard

$$
\text { Funding: } \begin{aligned}
& \$ 48,000 \text { (FY92) } \\
& \$ 59,300 \text { (FY91) }
\end{aligned}
$$

\section{Project Description}

This project explored the applicability of the precision sensing technology developed in the field of atomic force microscopy to miniaturization of accelerometers for use in seismological applications, in particular where small, borehole-operating sensors are required. The potential uses of the object devices are many, and, if the target specification can be reached, commercialization will be no problem. In fact, in the course of the development study, several potential manufacturers got wind of the work and volunteered to explore possible, cooperative, development programs.

The results of the two-year study, while very encouraging, are somewhat disappointing in that a robust, functioning prototype sensor was not produced. The goal performance remains feasible. The next problem will involve a serious effort in silicon microfabrication, under much cleaner environment than was available in the current exercise.

A survey of state-of-the-art displacement detectors and an analysis of the fundamental limitations on sensitivity as applied to seismometry led us to attempt the fabrication of a miniature torsion beam. While the results point out several problems, we are encouraged to believe that further efforts along indicated paths will result in a state-of-the-art miniaturized seismometer. 


\section{Accomplishments}

A resonant phase shift electronics system to provide force feedback and an output signal proportional to the restoring force was developed and tested. Also, an independent optical angular motion monitor was developed and tested to evaluate the performance of the miniature torsion beam displacement detector and control system.

We designed a torsion beam system to meet the requirements of a seisinic sensor. A number of dimensional parameters were considered, and the design shown in Figure 2 was constructed. The torsion beam is fabricated from silicon of dimensions $12 \mathrm{~mm}$ $\times 15 \mathrm{~mm} \times 0.381 \mathrm{~mm}$. The top capacitor plates are made from gold evaporated on the entire bottom surface of the torsion beam to a thickness of $\sim 1000 \AA$. These lower capacitor plates extend beyond the edge of the upper plate and serve as electrical leads $(0.5 \Omega)$ to the control system. The silicon used throughout the

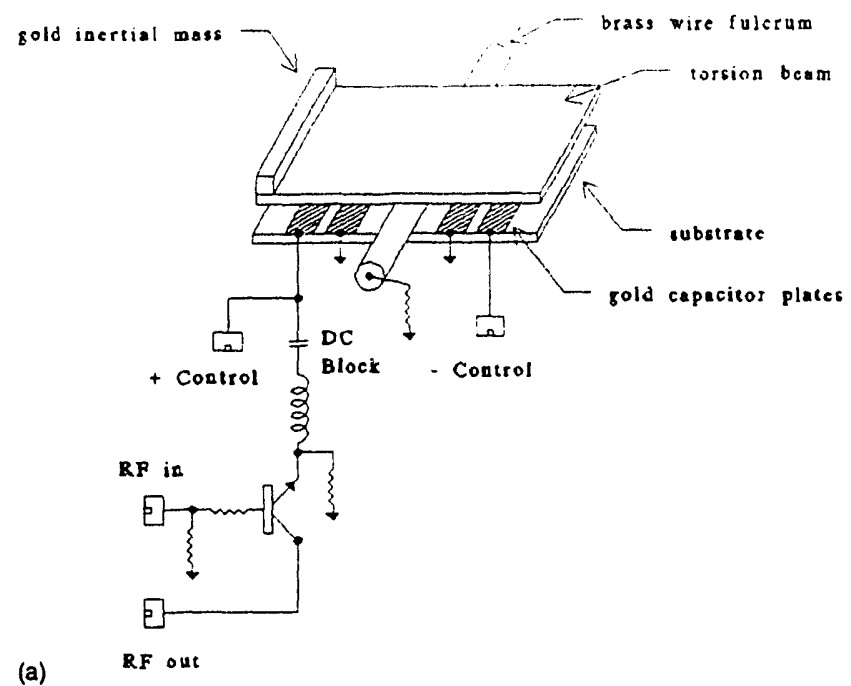

20 mm

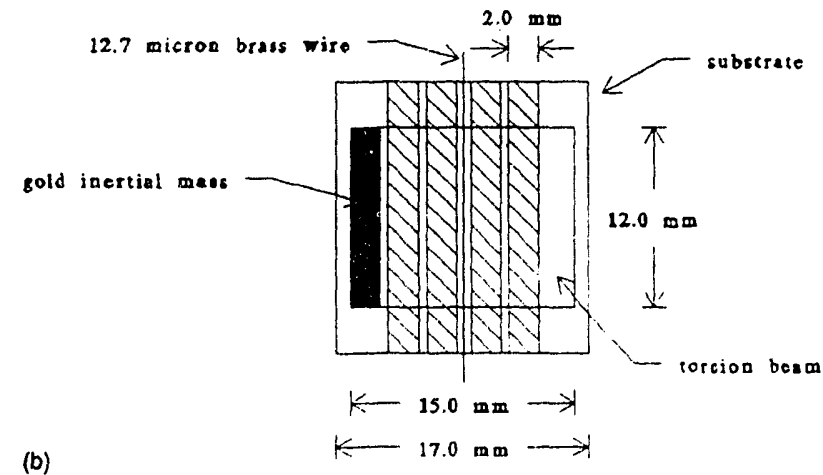

Fig. 2. (a) Torsion beam displacement detector and resonant phase shift circuit; (b) metallization pattern of torsion beam displacement detector. sensor is high-resistivity $(10,000 \Omega$-cm) material. The fulcrum/torsion fiber is brass wire that provides a capacitor gap of $12.7 \mu$. As a torsion beam the mechanical system has a mass of $0.18 \mathrm{~g}$ and a calculated resonant frequency of $1.2 \mathrm{sec}$.

This design is intended to develop a proof-of-principle test device. There is no doubt that this design would have shortcomings as a seismic sensor, but it will allow us to test various assembly. techniques and electronic designs.

The principal lesson learned in the mechanical assembly is the importance of maintaining particle-free surfaces between the top and bottom plate. The initial devices failed due to particle contamination that prevented free movement of the upper plate. The small gap and large area of this sensor make it particularly sensitive to particles.

While the capacitive displacement detector system provides a means of detecting and controlling the motion of the torsion beam, the actual magnitude of the beam motion can only be roughly estimated from the apparatus geometry. In order to provide a quantitative measure of the torsion beam motion, a heliumneon laser was employed to project a collimated light beam onto the surface of a small mirror attached to the top of the torsion balance assembly. After reflection from the mirror surface, the beam was directed onto a dual photodiode detector. Electrical signals from the two halves of the photodetector were amplified by low-noise transimpedance preamplifiers. They were then subtracted to produce a difference signal proportional to the beam position. This signal was calibrated with the use of a micrometer-driven translator on which the photodiode assembly was mounted. Small rotations of the torsion beam were therefore converted into easily measured beam translations at the detector surface.

It was not possible in this project to develop a completed inst ument; a number of conclusions were reached and suggestions can be made for further work. An analysis of the thermal contributions to sensor noise has shown that a successful seismometer can only be constructed if the criterion $\mathrm{MT}_{0} \mathrm{Q} \geq 1 \mathrm{~kg}$ $\mathrm{sec}$ is satisfied. While the precision and reproducibility of silicon fabrication technology can be used to overcome the mechanical instabilities of conventional miniaturized instruments of the Usher type, many of the designs fabricated to date do not meet this $\mathrm{MT}_{0} \mathrm{Q}$ criterion.

The torsion beam design of Miller et al. (Rev. Sci. Instrum. 62(3), 705 (1991)) appears to satisfy many of the requirements for a successful instrument if con- 
structional difficulties can be overcome. In our preliminary experiments, cleanliness and a dust-free assembly environment were found to be essential. A second critical element in the design is the torsion wire used to suspend the upper torsion beam. The micronsized carbon fiber and brass wires used by them and by us were a potential source of problems both in electrical conductivity and dissipative mechanical losses. Better performance is expected with cantilever suspensions fabricated from silicon, silicon nitride, or other material designs employing state-of-the-art silicon technology. Such design improvements are beyond the scope of this project but should be explored in future work.

An electronic control system employing resonant phase shift detection was constructed. The operation of this system was tested employing a varactor (electronically variable capacitor) to simulate the torsion beam capacitance. This instrumentation appears to be performing up to our expectations and should be wellsuited for future work. 


\section{Energy and Environment Division}

\section{X-Ray Absorption Spectroscopy}

Principal Investigators: Stephen P. Cramer, Jie Chen, Jan van Elp, J. Moore, and Xin Wang

Funding: $\$ 251,300$

\section{Project Description}

Our goal is to develop new types of $x$-ray spectroscopy. Three new techniques are under development: $x$-ray magnetic circular dichroism, site-specific $x$-ray absorption, and flow-pump-probe spectroscopy. Experiments are conducted at the National Synchrotron Light Source (at Brookhaven) and the Stanford Synchrotron Radiation Laboratory (at SLAC).

\section{Accomplishments}

$\mathrm{X}$-ray magnetic circular dichroism (XMCD) measures the difference in absorption of left and right circularly polarized $x$-rays by a sample in a magnetic field. We recorded the first $x$-ray magnetic circular dichroism spectrum of a paramagnetic sample, the Fe(III) center in the protein "rubredoxin" (see Figure 1). The effects are very large (about $40 \%$ absorption differences). The technique allows determination of the magnetic orientation of specific metal in different oxidation states and should have broad application to bioinorganic chemistry and magnetic materials science.

Site-specific $x$-ray absorption is based on small shifts in fluorescence energies that occur with oxidation state changes. We have demonstrated fluorescence shifts for $\mathrm{Mn}$ (II), $\mathrm{Mn}$ (III), and $\mathrm{Mn}$ (IV) (see Figure 2), and are now building spectrographs to allow similar studies on Fe and Ni compounds.

The technique of flow-pump-probe spectroscopy has in the past been used with a laser pump and millimeter-scale hard $x$-ray probe beams. We are currently building an apparatus to use micron-sized soft $x$-ray probe beams, along with a pulsed dye laser pump beam. Upon completion, the smaller probe size will allow significantly shorter time scales to be examined.

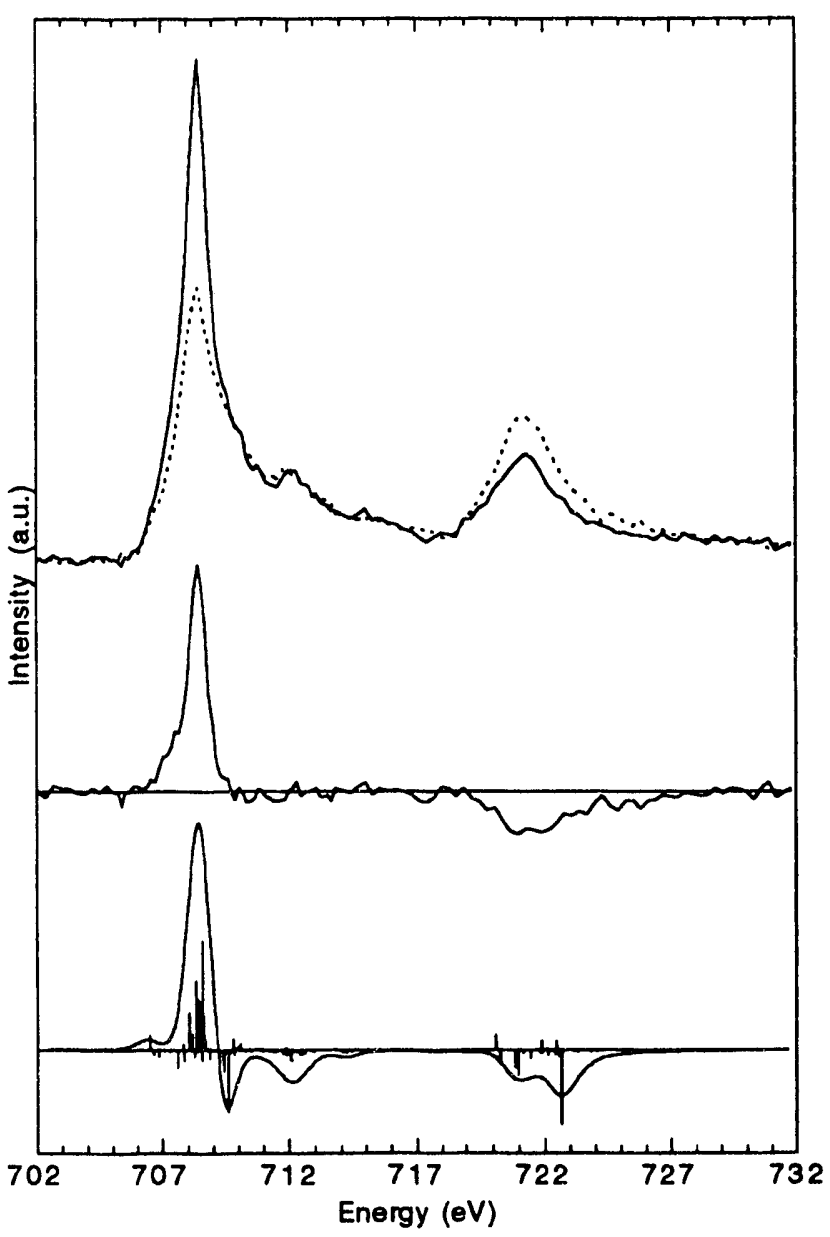

Fig. 1. The XMCD spectrum of $\mathrm{Fe}$ in rubredoxin.

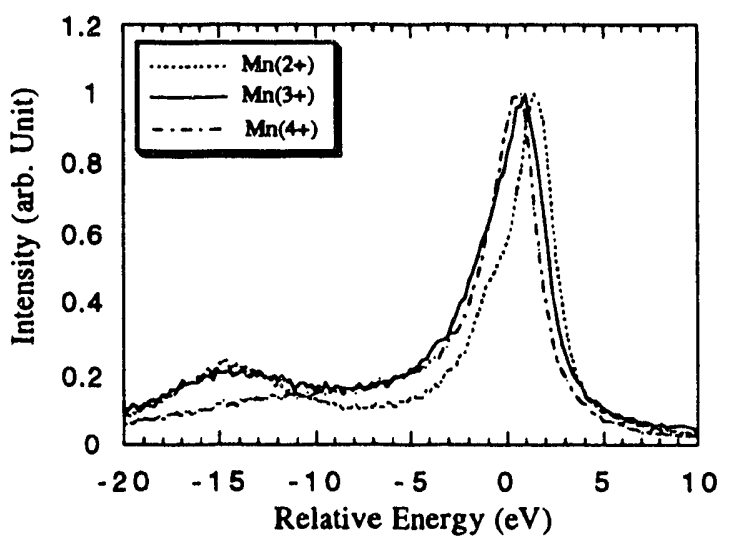

Fig. 2. Chemical shifts in manganese $K$ fluorescence. 


\section{Publications}

S.R. Hubbard, W.R. Bishop, P. Kirschmeier, S.J.

George, S.P. Cramer, and W.A. Hendrickson, "Identification and Characterization of Sites in Protein Kinase C," Science 253, 1776 (1991).

V. Stojanoff, K. Hamalainen, D.P. Siddons, J.B. Hastings, L.E. Berman, S.P. Cramer, and G.A. Smith, High-Resolution $X$-ray Fluorescence Spectrometer for Near-Edge Absorption Studies. Rev. Sci. Instrum., 63, 1125 (1992).

G.S. Waldo, O.C. Mullins, J.E. Penner-Hahn, and S.P. Cramer, Determination of the Chemical Environment of Sulphur in Petroleum asPhaltenes by X-ray Absorption Spectroscopy. Fuel 71, 53 (1992).

S.J. George, J. van Elp, J. Chen, Y. Ma, C.T. Chen, J-B. Park, M.W.W. Adams, B.G. Searle, F.M.F. de Groot, J.C. Fuggle, and S.P. Cramer, "L-edge X-ray Absorption Spectroscopy of Pyrococcus furiosus Rubredoxin," J. Am. Chem. Soc. 114, 4426 (1992).

S.P. Cramer, J. Chen, S.J. George , J. van Elp, J. Moore, O. Tench, J. Colaresi, M. Yocum, O.C. Mullins, and C.T. Chen, "Soft X-ray Spectroscopy of Metalloproteins Using Fluorescence Detection,"Nucl. Instrum. Meth. A 319, 285 (1992).

S. Mitra-Kitley, O.C. Mullins, J. van Elp, and S.P. Cramer, "Nitrogen Chemistry in Petroleum Asphaltenes and Coal by X-ray Absorption Spectroscopy," Fuel , in press.

S. Mitra-Kitley, O.C. Mullins, J. Chen, J. van Elp, S.J. George, C.T. Chen, T. O'Halloran, and S.P. Cramer, "Nitrogen Chemical Structure in DNA and Related Molecules by X-ray Absorption Spectroscopy, Biochem. Biophys. Acta, in press.

S. Mitra.Kitley, O.C. Mullins, J. van Elp, S.J. George, J. Chen, and S.P. Cramer, "Nitrogen in Petroleum Asphaltenes - Local Structure using FluorescenceDetected Soft X-ray Absorption Spectroscopy," J. Am. Chem. Soc. , in press.

K. Hamalainen, C-C. Kao, J.B. Hastings, D.P. Siddons, L.E. Berman, V. Stojanoff, and S.P. Cramer, "SpinDependent $\mathrm{X}$-ray Absorption in $\mathrm{MnO}$ and $\mathrm{MnF}_{2}$ Phys. Rev. $B_{\text {. , in press. }}$

J. van Elp, S.J. George, J. Chen, G. Peng, C.T. Chen, L.H. Tjeng, G. Meigs, H.J. Lin, Z.H. Zhou, M.W.W.
Adams, B.G. Searle, and S.P. Cramer, "Soft X-ray Magnetic Circular Dichroism - A New Probe for Studying Paramagnetic Bioinorganic Systems," submitted to Science.

S.P. Cramer, J. Chen, J. Christiansen, S.J. George, R. Tittsworth, B. Hales, and D. Coucouvanis, "EXAFS and L-edge Spectroscopy of Nitrogenase FeMo Protein, submitted to Molybdenum Enzymes, ACS Symposium Series, E. Stiefel, ed.

S.J. George, M.D. Lowery, E. Solomon, and S.P. Cramer, "Copper L-edge Spectral Studies: A Direct Experimental Probe of the Ground State Covalency in the Blue Copper Site in Plastocyanin," submitted to J. Am. Chem. Soc.

J. Chen, S.J. George, R. Tittsworth, B.J. Hales, J. Christiansen, D. Coucouvanis, and S.P. Cramer, "Iron EXAFS of A. Vinelanidii Nitrogenase Mo-Fe and V-Fe Proteins, submitted to J. Am. Chem. Soc.

\section{Simulation of an Advanced Utility- Customer Interface}

Principal Investigators: Donald F. Grether, Hashem Akbari, Arthur H. Rosenfeld, Pravin P. Varaiya, Felix F. Wu, and Carl Blumstein

Funding: $\$ 75,000$

\section{Project Description}

The electric utility industry stands on the threshold of a technological revolution. Within the next two decades, the transmission and distribution networks that deliver electric power to our homes and businesses will be paralleled by communications networks, and electric meters will be replaced by computers. This new information technology will transform the industry in three ways: (1) existing operations will become more automated; (2) the generation, transmission, and distribution facilities will be used more efficiently; and (3) a range of new energy services will be offered.

LBL and UCB house a significant portion of the nation's expertise in utility-customer energy manage- 
ment systems. Within the academic community, Berkeley is the leader in propagating the idea of a $3 \mathrm{C}$ (communication, computation, and control) system for utility automation. On the customer side, LBL is a national leader in pursuing energy efficiency technologies, particularly in the building sector, and has performed substantial research in evaluating potentials of energy management and control systems (EMCS) for energy efficiency improvement, utilitycustomer interface, real time pricing, and utility enduse monitoring.

The purpose of the project is to develop an LBL/UC program on advanced utility-customer communication, computation, and control (UC-3C) systems. The long-term goals are to

- Develop methodologies and computer facilities to simulate a b:road range of technological options and strategies.

- Use those '́acilities to compare technological options (e.g., for communication), investigate control and pricing strategies, and otherwise work toward optimal UC-3C systems.

- On the basis of such analyses, provide DOE and the utility community with unbiased information and later methodologies.

\section{Accomplishments}

The project needs to reflect the interests of the electric utilities, and DOE and other government agencies. To this end, the researchers regularly participate in relevant workshops and conferences, including:

- Information and Automation Technology Conference and Equipment Show, Electric Power Research Institute, June 26-28, 1991, Washington, DC [Akbari]

- Workshop on Real-Time Control of Electric Power Systems, Sponsored by the Department of Energy, November 19-21, 1991, Denver, CO [Grether]

- Second International Symposium on Distribution Automation and Demand Side Management, Sponsored by the Eureka Group and Electric Light \& Power, January 13-15, 1992, Fort Lauderdale, FL. [Wu]

In addition, researchers have undertaken an effort to better understand the various institutions' interests via a series of meetings with their representatives. During
FY 92 key meetings were held with the Electric Power Research Institute (EPRI) and the research arm of Pacific Gas \& Electric Company (PG\&E). The meetings with PG\&E have been particularly fruitful and have resulted in a planning process for a joint PG\&E/ $\mathrm{UCB} / \mathrm{LBL}$ effort.

The simulation work itself is based on the Ptolemy software environment, an object-oriented system developed at UCB. Programmed in $\mathrm{C}++$ and running on a UNIX workstation, Ptolemy is a very flexible framework, supporting heterogeneous system specification, simulation, and design. Each model of computation is called a "domain" and consists of an extensible library of functional blocks. The basic unit of computation in Ptolemy is the "block," represented graphically by an icon with terminals, corresponding to its "portholes." A block may be atomic ("star") or composite ("galaxy"). Applications are constructed graphically by connecting blocks. At runtime, a scheduler invokes the blocks; data are exchanged between the blocks in the form of "particles." Discrete units may be of several types: integer real complex or a general structure. Blocks may have "states," usersettable data structures that may be monitored from one execution to another. Ptolemy's strength and uniqueness lie in its capability of supporting the many aspects of UC-3C system modeling and simulation and the ease with which new application-specific design environments may be built.

The effort for FY92 involved first an object-oriented design of the UC-3C system, including object hierarchy, object definition, and methods, and secondly, the implementation of a feeder simulator. Line segments, switches, transformers, and capacitors are modeled as stars in the discrete-event (DE) domain. DE stars function as event-processing units that receives and process particles from the outside and generate output events after a user-given latency. Interconnection of stars constitute a feeder in a utility distribution system. To find the current state of the feeder, as well as the states in every component, a power flow calculation is necessary. Two implementations of power flow calculation have been completed. One is based directly on the DistFlow algorithm developed at UCB and the other is a distributed version of DistFlow developed as part of this project. The first version built a computational star to perform DistFlow and the second version uses message passing and local methods to calculate power flow. Work is underway to design Ptolemy stars to implement various customer response models. 


\section{New Approaches to Assessing Oceans as $\mathrm{CO}_{2}$ Sinks}

Principal Investigators: Ronald Ritschard, Kenneth Orvis, and Patrick Neale

Funding: $\$ 129,300$

\section{Project Description}

Critical uncertainties remain in our understanding of global biogeochemical cycling, particularly the carbon budget. Of the total estimated release of carbon dioxide due to fossil fuel burning and changing land use, less than half is now present in the atmosphere. The ocean is believed to be taking up much of the remainder at a rate of about $2 \mathrm{Gt} \mathrm{yr}^{-1}$; it is not known, however, how this capacity is regulated. Carbon. dioxide $\left(\mathrm{CO}_{2}\right)$ is the principal greenhouse gas, and there is little disagreement over the amounts of $\mathrm{CO}_{2}$ being released into the atmosphere. However, we remain unable to account for all the $\mathrm{CO}_{2}$ that is leaving the atmosphere and going into either terrestrial or oceanic sinks.

Oceanic primary productivity, especially the "new production" balanced by precipitation from ocean surface waters, is an important component of the global carbon budget. Recently, high-resolution measurements of variation in atmospheric oxygen have suggested that global oceanic new production may be at least twice as high as had been previously calculated based on estimates of ${ }^{14} \mathrm{C}$ uptake by marine phytoplankton. At the same time, early predictions that terrestrial standing biomass will increase in response to higher levels of atmospheric $\mathrm{CO}_{2}$ have been called into question by recent results suggesting that, in tropical forests at least, only the rate of carbon cycling will increase, while standing biomass remains unchanged. Oceanic fluxes may be the key. Knowledge of ocean and ocean-margin dynamics is essential to our ability to observe, analyze, and finally predict the slow response of climate to gradual increase in atmospheric greenhouse gas concentrations. The role of $\mathrm{CO}_{2}$ uptake by the oceans is controlled by seawater temperature, surface chemistry, and biological activity (photosynthesis, respiration, and production of detritus), and by mixing arid circulation at several scales. Many uncertainties remain in the dynamics of each of these major influences on open-ocean carbon fluxes as well as in others involved within ocean margins. To initiate a major effort on oceans research at LBL this project was begun to investigate several research topics related to the fate of $\mathrm{CO}_{2}$ in the oceans.
During the first year, these studies focused on three areas: (1) modeling atmospheric extinction and scattering at wavelengths critical to remote sensing of marine phytopigments to improve quantitative remote estimates of phytoplankton productivity, (2) reviewing the "iron limitation hypothesis," and (3) initiating laboratory studies to investigate the relationship between quantum yield of fluorescence and carbon fixation for the purpose of developing direct methods to remotely sense chlorophyll activity (primary production) in oceans. The preliminary results of these first year studies are briefly summarized below. In addition, we have initiated a pilot ocean margins project, designing a Geographic Information System (GIS) model of riverine discharge fluxes of carbon and nutrients into the ocean as functions of drainage basin and transport characteristics. The results from this model are very preliminary at this time and will not be discussed.

\section{Accomplishments}

1) Remote sensing of phytoplankton biomass and productivity offers an indispensable tool for understanding global rates of (and changes in) "new production" in the face of the dynamism and the vast extent of ocean ecosystems. Satellite-based and aircraft-based remote sensing instruments yield the only possible synoptic views of inherently dynamic aquatic systems. It is clear that new approaches for measuring oceanic prii-ary productivity are needed to address, especially at global scales, the discrepancy between existing estimates of $\mathrm{CO}_{2}$ uptake by marine phytoplankton and recent high-resolution measurements of variation in atmospheric oxygen. Of the several steps involved, our current work seeks to improve on two. This first project addresses remote recovery of the subtle visiblespectrum signatures relating to chlorophyll concentration and activity.

We have modeled the effects of relying on the standard atmospheric correction algorithms, under representative nonstandard conditions, using the Air Force Geophysics Laboratory's sophisticated Lowtran-7 model of atmospheric light interactions. Results thus far include some marked demonstrations of inaccuracies that result when aerosol effects fail to mimic those of aerosols encountered in marine profiles (Figure 3).

West Coast atmospheric conditions, especially during offshore airflow, often differ strongly from the marine standard. Closely related conditions are typical elsewhere (e.g., Peru, Namibia, Portugal, SW Australia), often associated with oceanic upwelling and resultant productivity maxima, and often, as in our California case, where obscuring advection fog almost 


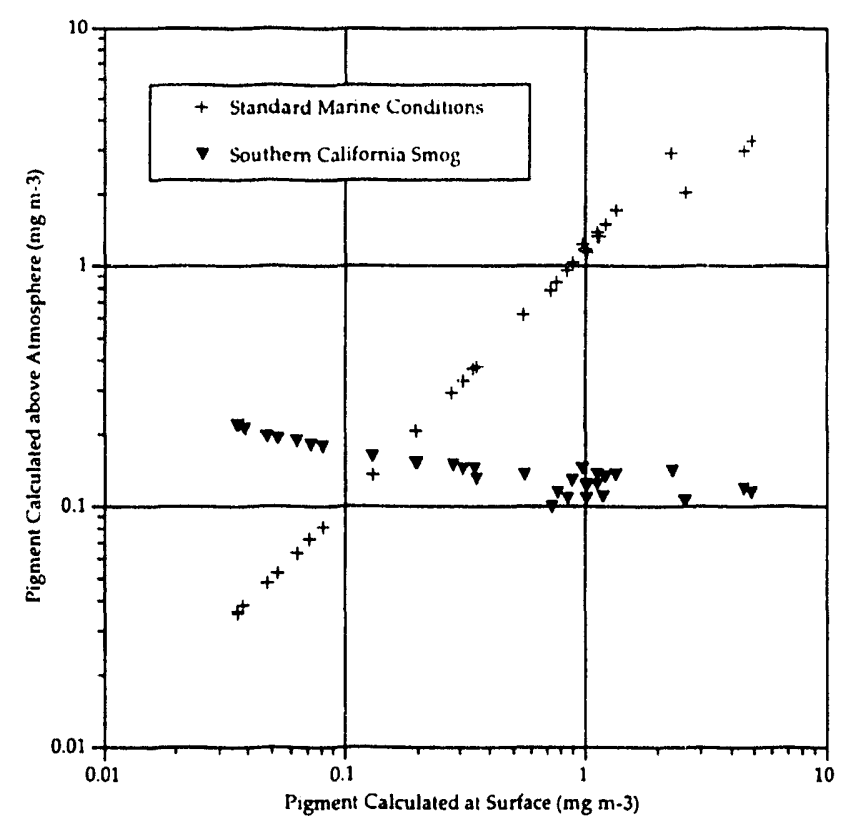

Fig. 3. Results of standard correction with two modeled atmospheres: a standard marine atmosphere (crosses) and a California summer atmosphere laced with urban aerosols (triangles), typical of urban vortex conditions over the Southern California Bight. Both were calculated using the surface-flux data set of Clark as input.

always coincides with marine atmospheric profiles. We have modeled a variety of West Coast conditions, and are also analyzing effects of multiple scattering at large solar zenith angles. Results will be tested with archived CZCS and AOCI imagery and with $\mathrm{CalCoFi}$ and other sea-truth data. Our findings are pertinent elsewhere and will also prepare us for meeting SeaWiFS accuracy standards, particularly in West Coast waters.

2) The "iron limitation hypothesis" proposed in 1990 suggests that low concentrations of surface nutrients, especially iron, limit the biological activity of ocean phytoplankton in polar oceans, and thus the atmospheric drawdown of $\mathrm{CO}_{2}$. According to this hypothesis, if sufficient iron were added to the surface waters in affected areas, a de novo $\mathrm{CO}_{2}$ sink would result. We prepared a paper on the use of marine algae as $\mathrm{CO}_{2}$ sinks. We have also been invited to write and present a paper on oceanic disposal of $\mathrm{CO}_{2}$ at the IEA Carbon Dioxide Disposal Symposium at Oxford University, U.K. in March of 1993.

3) Our second study that seeks to improve the measuring of ocean productivity addresses the remote sensing of chlorophyll activity as opposed to chlorophyll mass. A possible new approach is to monitor phytoplankton processes through the associated emission of fluorescence by chlorophyll a. Most of the physiological processes that regulate photosynthesis also have some effect on fluorescence. As an initial step to developing fluorescence-based approaches for measuring ocean primary production, we initiated laboratory experiments to study the fluorescence characteristics of selected phytoplankton species under varying light conditions.

In these experiments the znarine diatom Thalassiosira pseudonana (clone $3 \mathrm{H}$ ) was grown in an artificial sea water medium under a continuous illumination of $\mathbf{2 0 0}$ $\mu \mathrm{mol} \mathrm{m} \mathrm{m}^{-2} \mathrm{~s}^{-1}$ at $20^{\circ} \mathrm{C}$. Phytoplankton fluorescence characteristics were measured with a pulse amplitude modulated (PAM) fluorometer with fiber optic probe directed at the surface of a $200 \mathrm{ml}$ aliquot of algal culture. Ambient irradiance was provided by a 1000 $\mathrm{W}$ quartz halogen lamp (high irradiance) or $200 \mathrm{~W}$ incandescent floodlamp (low irradiance). Treatment irradiance (400-700 $\mathrm{nm})$ was measured in the sample by an immersed quantum scalar irradiance sensor fitted with a $4 \pi$ collector. In our preliminary set of experiments, we have tested the time resolution of the PAM fluorometer in connection with establishing the time course of fluorescence change over a range of light intensities.

Previous studies with terrestrial plants have suggested that the ratio of $\left(F_{m}-F_{s} / F_{m}\right)$ is proportional to changes in the quantum yield of the photosystem (PSI) electron transport in plant photosynthetic apparatus. the coupling between PSII quantum yield and photosynthesis in higher plants has led to the suggestion that photosynthesis can be predicted from PSII fluorescence yield. The phytoplankton measurements thus far are consistent with this suggestion. A relative estimate of photosynthesis (the product of irradiance and relative PSII quantum yield) is a hyperbolic function of irradiance which saturates around 300 $\mu \mathrm{mol} \mathrm{m} \mathrm{m}^{-1} \mathrm{~s}^{-1}$ (Figure 4). This is reasonable for a culture grown at $200 \mu \mathrm{mol} \mathrm{m} \mathrm{m}^{-2} \mathrm{~s}^{-1}$. However, rates of carbon incorporation are needed to confirm this observation. These will be measured in the next set of experiments using fluorescence change.

Using a PAM fluorometer or similar approach, we may be able to "ground truth" how fluorescence yield and photosynthesis vary between surface water and deeper in the water column. This would dramatically improve our ability to estimate photosynthesis from solar-stimulated fluorescence detected, for example, by the ocean-color remote sensors discussed above. 


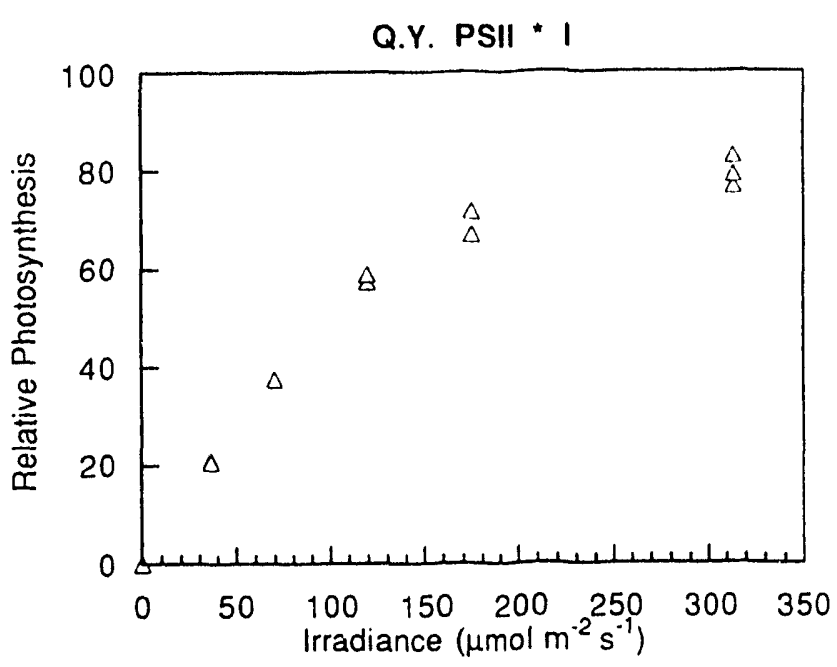

Fig. 4. Relative photosynthetic rates of Thalassisira pseudonana, estimated from PAM fluorometer measurements, as a function of irradiance. Photosynthesis was estimated as a product of irradiance and the relative quantum yield (Q.Y.) of PSII. Note irradiance is on a linear scale.

\section{Publications}

P.J. Neale and R.L. Ritschard, "Study of Marine Phytoplankton Fluorescence-Photosyn thesis Relationship," in preparation.

K.H. Orvis and R.L. Ritschard, 1992, "Reanalysis of CZCS Images Acquired under West Coast Continental and Urban Air Mass Regimes," in preparation.

R.L. Ritschard, "Marine Algae as $\mathrm{CO}_{2}$ Sink," Water, Air, and Soil Pollution 64, 289 (1992).

R.L. Ritschard, "Oceanic Disposal of $\mathrm{CO}_{2}$ " to be presented at IEA Carbon Dioxide Disposal Symposium, Oxford University, U. K., March 29-31, 1993.

\section{Short-Wavelength Materials: GaN and $\mathrm{SiC}$}

Principal Investigator: Mike Rubin

Funding: $\$ 75,000$

\section{Project Description}

The purpose of this project is to develop wide-band gap semiconductors and devices for photonic and electronic applications. These applications include blue or ultraviolet LEDs and lasers, and photovoltaics, as well as high-power, high-frequency, or hightemperature electronics. Our initial goal was to deposit crystalline thin films of GaN and other materials such as $\mathrm{SiC}$ and $\mathrm{AlN}$. GaN is of special interest because it has a direct band gap ( $3.4 \mathrm{eV})$ and it is completely miscible and vacuum compatible with other III-V nitrides such as AlN and InN. Both SiC and AlN can be used as buffer layers for $\mathrm{GaN}$. Our longer term objective is to produce p-type $\mathrm{GaN}$ that can then be used to make efficient devices.

The strong chemical bonds in GaN have many advantages in terms of properties, but make defect-free buik or epitaxial material difficult to obtain. Furthermore, because of the low-solubility and high-equilibrium vapor pressure of nitrogen, $\mathrm{GaN}$ tends to form with high concentrations of nitrogen vacancies. Poor lattice and thermal-expansion match with practical substrates cause other types of defect generation. We approached these problems by sputtering films at very highnitrogen pressures and by using AlN buffer layers on sapphire and GaAs. Even better films will be produced with an ion-assisted deposition process. The best films will be doped with $\mathrm{Mg}$ to produce $\mathrm{p}$-type conduction. The simple picture of the nitrogen vacancy as a shallow donor and $\mathrm{Mg}$ as a shallow acceptor in $\mathrm{GaN}$ is inadequate. Despite the increasing interest in this material, no studies have thoroughly characterized the defects using modern methods. We are applying several techniques to this problem including DLTS (deep level transient spectroscopy), EPR (electron paramagnetic resonance), ODMR (optical/luminescence detected magnetic resonance), TEM (transmission electron microscopy), PL (photoluminescence) and temperature-dependent Hall measurements.

\section{Accomplishments}

We have produced crystalline $\mathrm{GaN}$ on single-crystal substrates of sapphire, GaAs and $\mathrm{Si}$. Figure 5 shows that our films have a narrow range of epitaxy on sapphire between 660 and $685^{\circ} \mathrm{C}$. Peak widths in PL and XRD are slightly narrower and band gap a little higher than reported for most other thin films. We also grew buffer layers of AlN by sputtering. Scanning electron micrographs showed a significant decrease in surface roughness with the buffer layer as well as larger more regular grains.

Early attempts at thin-film growth resulted in electron concentrations greater than $10^{19} \mathrm{~cm}^{-3}$. P-type material was produced only recently (by others) starting with $\mathrm{n}=2 \times 10^{16} \mathrm{~cm}^{-3}$ and $=8 \mathrm{~cm}^{2} / \mathrm{V}$-s. The lowest carrier concentration of $\mathrm{n}=8 \times 10^{13} \mathrm{~cm}^{-3}$ was obtained (also by others) by the technique of ECR (electron cyclotron 


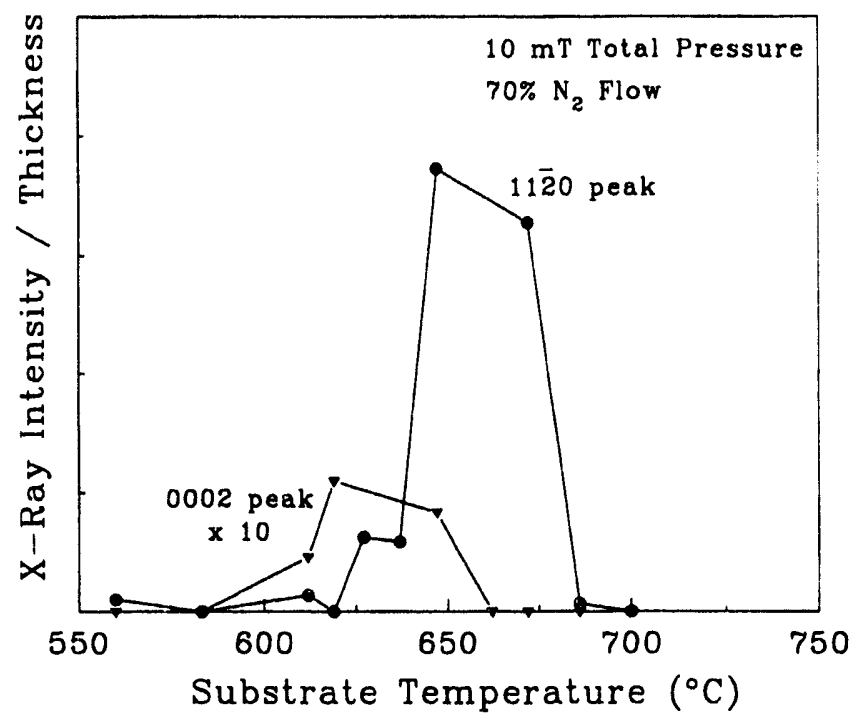

Fig. 5. Effect of varying growth temperature on $x$-ray peak intensities of sputtered GaN films.

resonance)-assisted MBE (molecular beam epitaxy). Our best sputtered film had $\mathrm{n}=8.4 \times 10^{14} \mathrm{~cm}^{-3}$ and a mobility of $327 \mathrm{~cm}^{2} / V$-s from room-temperature Hall measurements. The four-point resistivity decreases by several orders of magnitude over a narrow range of growth temperature. This trend is indicative of material with vacancy defects, presumably nitrogen vacancies in this case. We completed modifications to an existing vacuum deposition system to allow a modified form of MBE growth. An ion source was added to provide a high flux of activated nitrogen species at the growing film. This should result in films of even better quality. Efforts will continue to incorporate $\mathrm{Mg}$ by both ion implantation and by direct evaporation.

\section{Publications}

J. Ross, M. Rubin, and T.K. Gustafson, "Crystalline Growth of Wurtzite GaN on (111) GaAs," Mat. Res. Soc. Symp. Proc. 242, 457 (1992).

N. Newman, J. Ross, and M. Rubin, "Thermodynamic and Kinetic Processes Involved in the Growth of Epitaxial GaN Thin Films," submitted to Appl. Phys. Lett., LBL-32876. 


\section{Engineering Division}

\section{Custom Gallium Arsenide Integrated Circuit Design for Applications in Nuclear and Particle Physics}

Principal Investigators: Stuart A. Kleinfelder, Oren Milgrome, and Issy Kipnis

Funding: $\$ 27,400$

\section{Project Description}

This project seeks to develop the capability to design custom microwave speed GaAs integrated circuits. The research initiated explorations into the techniques of GaAs design, necessary computer-aided design tool integration, forging part fabrication pathways, and gaining experience with very high-speed part testing. A specific goal is to proceed to the point of actually fabricating and validating one or more significant experimental $\mathrm{GaAs}$ integrated circuits. The design projects included an amplifier, a comparator, a medium-scale digital circuit, and GaAs and ECL-level compatible receivers and transmitters. This investment would provide a basic, proven foundation for future designs.

\section{Accomplishments}

To demonstrate basic competency in digital design and to test the speed potential of digital circuits in comparison with silicon devices, a 32-bit full adder circuit was designed. The propagation delay of the carry ripple in such an adder is a traditional real-world demonstration of the speed potential of a new technology. In addition, this part will prove the performance of the ECL-compatible receivers and 50-ohm output buffers included. This device has been fabricated and delivered to LBL.

Extending the scope of the digital test circuits, a design that is close to being a viable product was created: a programmable-skew clock transmitter. In many applications, a physically dispersed system of many elernents must be accurately synchronized to a master clock. A traditional way of accomplishing this is to use accurately matched cable delays. Today's detectors are large, fast, and complicated, with many thousands of such delays needed. A programmable delay would have the benefit of allowing the timing to be adjusted after construction. GaAs is an ideal technology for such a device, due to its speed and hence the high-resolution timing possible, and due to the relative ease of achieving very low-output impedances for good clock drive. The implementation included an ECL-level receiver, a 32-tap active delay line with tap output multiplexing, a 5-bit by 32-word read-only memory decoder, and low-output impedance driver. A master clock enters the chip and propagates down the active delay line. An externally applied 5-bit word selects the tap, and hence the delay, from which the propagating clock will be extracted. The 5-bit resolution, at a projected 250 ps per tap, results in a programmable delay spanning about $8 \mathrm{~ns}$. A more useful chip would be larger and have a longer range and multiple independently programmable outputs.

In order to gain some experience with analog circuits in GaAs, a fast amplifier chip was designed and fabricated, containing six channels of single-ended amplifiers in three different configurations. The amplifiers consist of cascoded gain stages with a follower output buffer and on-chip charge integrating feedback. Fall times of a few nanoseconds are predicted by our circuit simulations given a $10 \mathrm{pF}$ detector and a $10 \mathrm{pF}$ output load; much faster than any of our previous silicon designs. Individual transistors for device characterization and modeling were also included.

\section{Development of a Capability for the High-Density Packaging of High- Frequency Integrated Circuits}

Principal Investigators: Jacques E. Millaud, Robert P. Ely, Carl H. Haber, Michael E. Levi, Stuart A. Kleinfelder, and Oren B. Milgrome

Funding: $\$ 60,000$

\section{Project Description}

The development of complex detector systems, in particular but not exclusively, for high-energy physics or nuclear science, imposes the severe constraints of 
high-signal frequencies, small physical area, and lowpower dissipation on the associated electronics. Tne performance levels in future experiments will require the assembly of bare silicon circuits on a silicon or ceramic intercunnection substrates with micron-sized features to reduce capacitances and line lengths. This high-density interconnection technology appears to have matured to commerciai feasibility. The use of this technology may be reciuired for current silicon tracker and calorimeter electronics realization, and will be mandatory for increased detector speeds and densities, such as proposed for the $B$ factory. The demonstration of the high-density, high-frequency, multi-chip module proposed will improve current detecto- designs, and remove significant engineering constraints on future experimerts.

\section{Accomplishments}

The review of packaging technologies for Multi-Chip Modules dici show that most of LBL needs can be met using multilayer board laminates with fine line pitch ( $<6$ mils). This technology offers reasonable heat dissipation capability, low-cost prototyping, NonRecurring Engineering (NRE) costs of $\sim \$ 1500$ lowproduction costs, and short turnaround time (a few weeks). Ceramic-based substrates (hybrids) do not offer a comparable packaging density. They are more expensive to design, prototype, and produce; turnaround times are substantially longer. Thermal behavior is somewhat better than laminates. Silicon boards offer the highest trace density (2 mils pitch, 1 mil vias) excellent heat dissipation characteristics. However, their limited layering capability and their currently very high prototyping costs-NRE of $\sim \$ 70,000-$ severely affect their usability. Production costs are also quite high.

Currently used layout tools are more than adequate for known projected designs. Customization of libraries and process files is simple. Interfaces to fabrication facilities are readiiy available. A survey and evaluation of extraction/modeling tools have been made: OEA (Metal, Henry, PG plane), Quad Design (PDQ), TMA (Raphael), and PACIIIC NUMERIX.

OEA's tool set has been procured as well as an interface with layout tools. OEA's tools are currently being used for MCM designs, printed circuit designs, and integrated circuit layout design.

As a test of the technology capability, an existing hybrid circuit on ceramic substrate has been relayed out into a fine pitch board laminate technology. The new layout shows space and mass savings while providing substantial savings in layout time and prototyping/production costs.

\section{Extended $X$-Ray Absorption Fine Structure Spectroscopy (EXAFS) in the 1-4 keV Region}

Principal Investigators: Carolyn S. Rossington, Kin Man Yu, Wladyslaw Walukiewicz, and Joseph M. Jaklevic

Funding: $\$ 75,500$

\section{Project Description}

This project developed methods for perforning fluorescence extended $x$-ray absorption fine structure spectroscopy (EXAFS) in the $1-4 \mathrm{keV}$ region. There are a number of low $Z$ elements of significant materials and biological interest with absorption edges in this region, but which have not been easily accessible for EX^ FS study due to the experimental difficulties associated with low-energy $x$-ray attenuation and detection. A new $x$-ray spectrometer was to be designed and built at LBL, specifically optimized for low energy EXAFS work. The semiconductor materials systems of $\mathrm{CdTe}: \mathrm{Cl}$ and $\mathrm{ZnSe}: \mathrm{Cl}$ were chosen for initial study due to their importance in II-VI semiconductor research and to demonstrate the feasibility of the lowenergy EXAFS technique. Initial EXAFS measurements were to be performed at the Stanford Syrichrotron Radiation Laboratory (SSRL), and the resulting technology and expertise are intended to open an area of research applicable to the $x$-ray energy range provided by bending magnet beamlines at LBL's Advanced Light Source synchrotron.

\section{Accomplishments}

The materials of interest include $\mathrm{Cl}$-implanted $\mathrm{CdTe}$ and $\mathrm{ZnSe}, \mathrm{Mn}$-implanted $\mathrm{GaP}$, and $\mathrm{Mn}$ in $\mathrm{CdMnTe}$ alloy. The materials were thoroughly characterized using such techniques as Hall effect measurements; secondary ion mass spectroscopy; Raman spectroscopy; and proton-induced $x$-ray emission to elucidate information concerning the electrical activity, doping levels, and atomic bonding. The EXAFS $x$-ray spec- 
trometer was designed, built, and tested at LBL. The spectrometer was built in two stages, to facilitate the testing of the new design for EXAFS work. The spectrometer in its first stage had an energy resolution suitable for the study of the Mn-doped materials, but the improved resolution offered by the second stage of the spectrometer was required for the EXAFS measurements of the $\mathrm{Cl}$-doped materials.

EXAFS measurements of the $\mathrm{Mn}$-implanted $\mathrm{GaP}$ and $\mathrm{Mn}$ in CdMnTe alloy were performed at SSRL during June 14-23, 1992 and August 6-12, 1992, using the spectrometer in its first stage. High doses of $\mathrm{Mn}$ ions $\left(2.5 \times 10^{16} \mathrm{~cm}^{-2}\right)$ were implanted into single crystals of $\mathrm{CdTe}$ forming thin films of magnetic $\mathrm{Mn}_{x} \mathrm{Cd}_{(1-x)} \mathrm{Te}$ ailoy (maximum $x=0.2$ ). The materials were then annealed under various conditions. The EXAFS results on this material show that the Mn atoms in the implanted and implanted/annealed samples have local environments similar to bulk-grown CdMnTe crystals. While the Mn atoms in the implanted samples have a nearest neighbor distance similar to those of the bulk alloy and implanted/annealed samples, the next nearest neighbor distance is significantly larger, but becomes comparable again following annealing. A typical EXAFS spectrum of the $\mathrm{Mn}_{0.2} \mathrm{Cd}_{0.8}$ Te material is shown in Figure 1(a), and the Fourier Transform of that spectrum revealing the nearest neighbor distances is shown in Figure 1(b). The local structure of $\mathrm{Mn}$ in the implanted and implanted/annealed GaP materials showed significant differences, but additional EXAFS measurements are required to determine the exact nature of the $\mathrm{Mn}$ environments.

After the August EXAFS measurements of the Mndoped CdTe were completed, the spectrometer was upgraded to its second stage and testing at LBL showed that it had the improved energy resolution required for the EXAFS study of the Cl-doped materials.

\section{Publications}

K.M. Yu, J.W. Ager III, N. Derhacobian, R. Giauque, J.M. Jaklevic, C.S. Rossington, W. Walukiewicz, and M. Wesela, "EXAFS Analysis of Ion Beam Synthesized CdMnTe Thin Films," presented at the 19th SSRL Users Meeting, October 21-23, 1992, Stanford, CA, and paper in progress.

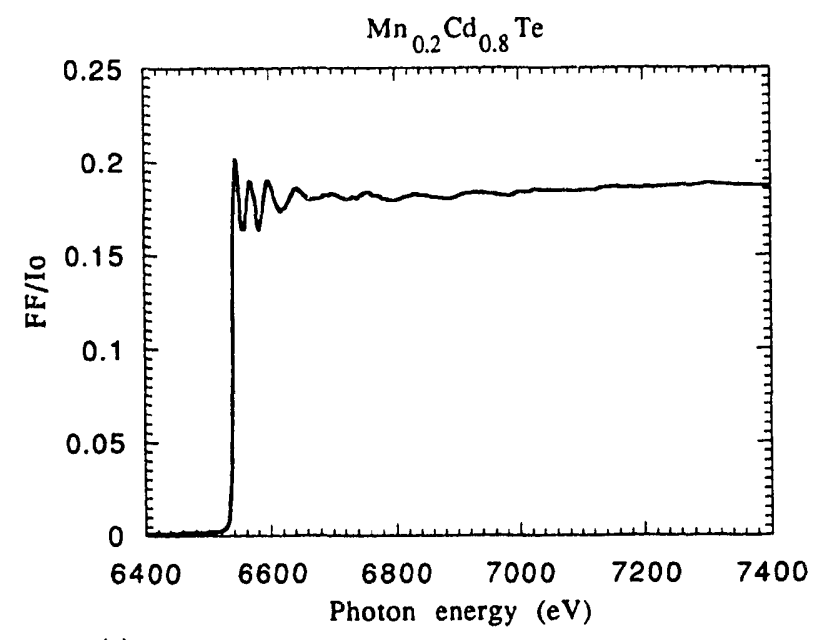

(a)

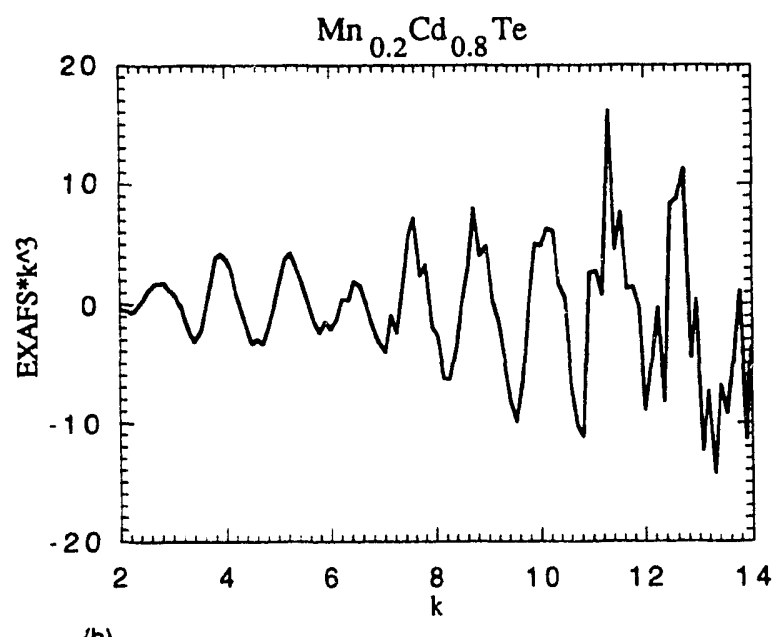

(b)

Fig. 1. (a) EXAFS fluorescence intensity as a function of excitation photon energy for a typicai $\mathrm{Mn}_{0.2} \mathrm{Cd} d_{0.8} \mathrm{Te}$ material; (b) Fourier transform of the spectrum in (a) revealing the nearest neighbor distances for the Min local environment.

K.M. Yu, J.W. Ager III, N. Dishacobian, R. Giauque, J.M. Jaklevic, C.S. Rossington, W. Walukiewicz, M. Wesela, and P. Becla, "EXAFS Analysis of Magnetic Semiconductor Thin Films Synthesized by Ion Beam Technique," to ve presented at the 1993 Materials Research Society Spring Meeting, San Francisco, CA, April 12-16, 1993. 


\section{Environment, Health \& Safety Division}

\section{Modification of an Andersson- Braun-Type Remmeter to Extend Its Sensitivity to $400 \mathrm{MeV}$}

\section{Principal Investigator: Rai-Ko S. Sun}

Funding: $\$ 98,900$

\section{Project Description}

A remmeter is an instrument that measures the absorbed dose in tissues (dose equivalent) from a radiation field. The Andersson-Braun (A-B) type of detector is the most commonly used remmeter for monitoring neutron dose equivalent in the energy range from $0.025 \mathrm{eV}$ to $10 \mathrm{MeV}$. The range limitation of current remmeters, which do not measure neutron dose equivalents above about $15 \mathrm{MeV}$, is a serious problem at high-energy accelerator facilities, where a much wider range of neutron energies exists. The purpose of this project was to simulate the responses of the A-B remmeter for medium- and high-energy neutrons ( $>10 \mathrm{MeV}$ ) using the Monte Carlo codes LAHET and MCNP. By introducing a lead sleeve surrounding the $\mathrm{BF}_{3}$ detector, the remmeter response at high energies will be enhanced based upon the $\mathrm{Pb}(n, \mathrm{xn})$ reactions. The goal of this project is to verify the calculations experimentally and to convert the instrument to a new and highly valuable measurement tool for nuclear science and high-energy physics.

\section{Accomplishments}

Several experimental models of A-B remmeters, all cylindrical in shape (Figure 1), were designed and constructed to have moderators of lead $(\mathrm{Pb})$ or polyethylene $\left(\mathrm{CH}_{2}\right)$, both of which are interchangeable inside the same remmeter. The theoretical Monte Carlo calculations for neutron energies from $1 \times 10^{-8} \mathrm{MeV}$ to $2000 \mathrm{MeV}$ have been completed, for the models with both $\mathrm{Pb}$ and $\mathrm{CH}_{2}$ moderators. Experiments were carried out mainly with monoenergetic neutron beams in the high-energy range, from $20 \mathrm{MeV}$ to $1 \mathrm{GeV}$. The models were tested at 7 energies: at LBL, $40 \mathrm{MeV}$ at the 88-Inch Cyclotron, and 400 and $1050 \mathrm{MeV}$ at the Bevalac; at UC Davis, 23, 42, 50, and $65 \mathrm{MeV}$. The results for the response of the modified and standard

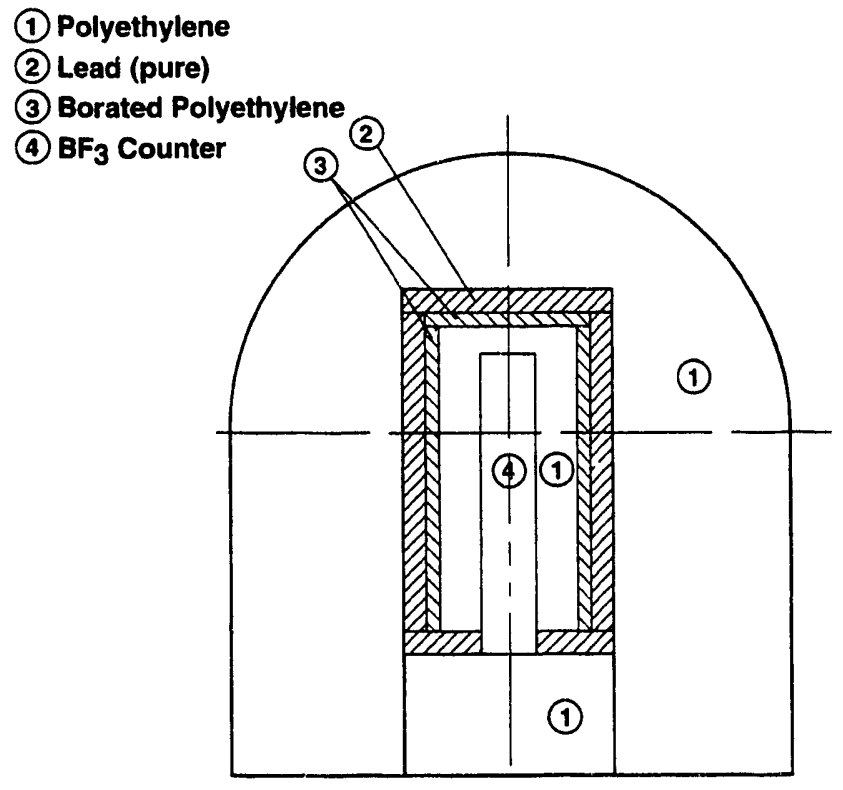

Fig. 1. Modified Andersson-Braun remmeter.

A-B remmeters are expressed as the ratio of the response of the remmeter with the lead-containing moderator to that of the remmeter with the polyethylene moderator. Experimental results are in good agreement with the calculated values, well within $12 \%$. Figure 2 shows the theoretical calculation values and

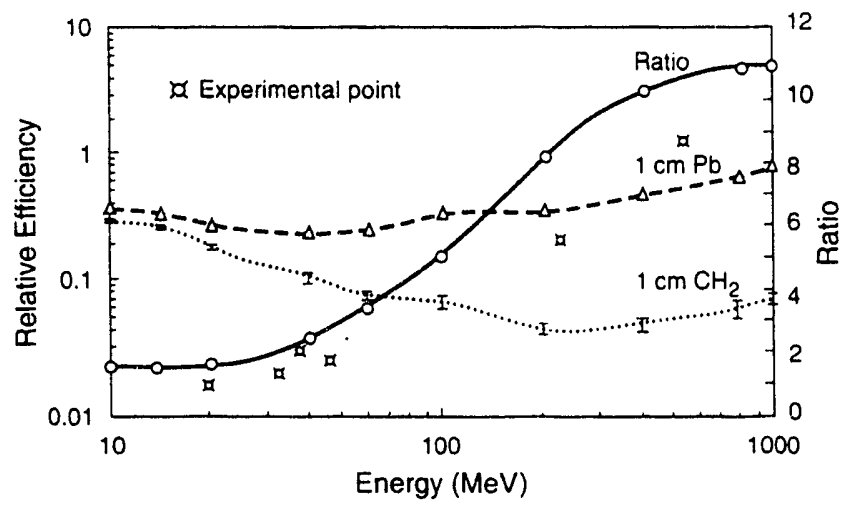

Fig. 2. Responses of modified Andersson-Braun remmeter. 
the experimental results. The theoretical calculations for energies above $10 \mathrm{MeV}$ up to $1 \mathrm{GeV}$ have thus been verified experimentally.

The preliminary results show that the development of a remmeter that will operate in the high-energy range can be achieved, and the Monte Carlo method using the LAHET and MCNP codes proves to be a powerful and reliable tool for the design of a new instrument. Further experiments will be carried out at energies both above and below those reported in this paper. In future work, various modifications to the standard A$B$ remmeter will be tried, and the responses of these remmeters to neutrons will be adjusted to match the dose equivalent curve as required.

In the course of development, it has been found that the experimental model is directionally dependent, with a deviation of about $15 \%$, between the radiations along the cylindrical axis and perpendicular to the axis. Based upon a theoretical investigation using the Monte Carlo method, a new remmeter with a spherical shape would solve the problem of directivity. This model will be a brand-new type of neutron remmeter, which is broad-band in energy, compact, and portable, that would be available for technology application for industries or research institutes within a few years. A patent application is pending.
This research project is also of interest to the Institute of High Energy Physics (IHEP), the People's Republic of China (PRC), and has been chosen by the PRC/USA Joint Committee on High Energy Physics, at the 12th Meeting, October 30-31, 1991, as one of the cooperative programs with LBL on radiation monitoring systems.

\section{Publications}

R.K. Sun, "Modification of an Andersson-Braun-Type Remmeter to Extend the Sensitivity to $400 \mathrm{MeV}$," presented at Lawrence Berkeley Laboratory, April 17, 1992.

R.K. Sun, G.F. Krebs, A.R. Smith, and H.H. Hsu, "A Neutron Dose Detector With REM Response to 1 $\mathrm{GeV}$," presented at the ANS/ENS 1992 International Conference, Chicago, Illinois, November 15-20, 1992.

R.K. Sun, and H.H. Hsu, "Simulation of the Response of a Modified Andersson-Braun Neutron Remmeter," to be published in Health Physics Journal.

\section{Patent}

A neutron Remmeter with Sensitivity Extended to Energy Range of $1 \mathrm{GeV}$. (pending). 


\section{Information and Computing Sciences Division}

\section{Robust Shotgun DNA Sequence Assembly}

Principal Investigators: Frank Olken, Eugene L. Lawler, and Dariel M. Gusfield

Funding: $\$ 82,900$

\section{Project Description}

We are developing algorithms for DNA sequence assembly from shotgun data that address common sources of difficulty for current systems: repetitive DNA sequences, chimeric clones, and nonuniform error rates along fragments. Our work builds upon the four-phase approach of our collaborator J. Kececioglu and E. Myers O. Kececioglu, "Exact and Approximation Algorithms for DNA Sequence Reconstruction," Ph.D. thesis, CS Dept., Univ. of Ariz., 1991), which decomposes sequence assembly into overlap detection, fragment orientation, fragment layout, and consensus sequence determination.

\section{Accomplishments}

Most of our efforts have been concerned with data characterization, algorithm design, and acquisition of datasets and codes that we will modify or incorporate into a new sequence assembly system. We have obtained sample sequencing data from our collaborators T. Hunkapiller and W.-Q. Chen, as well as a copy of the Kececioglu-Myers sequence assembly code (and user interface FAB). Coding, integration, and testing will occur in FY 93.

Current sequence assembly codes are confounded by repetitive DNA sequences, such as Alu and L1 repeats. Whereas these codes tend to stack or arbitrarily permute fragments that sample copies of a repeat, we intend to separate such fragments by screening against databases of prototypical sequence repeats. We have obtained such a repetitive sequence database from J. Jurka. Preliminary analysis and experiments indicate that by multiple sequence alignment against represen- tative consensus sequences of repeat families, fragments that sample different copies of a repeat may be distinguished, even when the error rate for approximate repeats approaches the sequencing error rate. We envision that this would be done during the overlap detection phase.

Also, our collaborators G. Alexander and T. Speed have devised a maximum likelihood-based method for overlap detection and scoring, which accounts for substitution errors (but not repetitive DNA).

Chimeric clones that combine dispersed fragments can cause incorrect melding of regions, and so prevent closure of contigs. We are investigating three approaches for screening chimeric clones: interrupted fragment alignments, likelihood tests on overlap graph confornations, and local violations of interval graph axioms.

Current methods for fragment orientation and layout either use heuristics, which allow these phases to be combined, or use optimization methods, which force the phases to be considered in isolation. We are implementing an orientation and layout algorithm based on graph matchings that combines both phases while applying optimization methods. We have obtained a matching code (from $\mathrm{H}$. Gabow) that we are incorporating into the Kececioglu-Myers code.

A two-pass strategy is being examined in conjunction with this algorithm. The first pass solves orientation and layout using only highly reliable overlaps with the low-error ends of fragments not containing known repeats. The second pass closes the resulting contigs by incorporating less reliable overlaps.

Improved consensus sequence determination is based on new algorithms for multiple sequence alignment $\sigma$. Kececioglu, "The Maximum Weight Trace Problem in Multiple Sequence Alignment," Technical Report, Computer Science Department, University of California, Davis, 1992), combined with generalized maximum likelihood "voting" methods (ChurchillWaterman, Genomics, Sept. 1992), and based on position- and sequence-dependent error models being developed (and calibrated) by our collaborators $T$. Hunkapiller, W.-Q. Chen, G. Alexander, and T. Speed. 


\section{Life Sciences Division}

\section{Analysis of a Cell Cycle Regulatory Gene}

Principal Investigator: Judith Campisi

Funding: $\$ 100,100$

\section{Project Description}

The long-term goal of this project was to understandat a molecular level-how cdc2/p34, a critical cell cycle regulatory gene, is controlled during normal cell proliferation and in preneoplastic and tumor cells. $\mathrm{cdc} 2 / \mathrm{p} 34$ encodes a protein kinase that is essential for cell growth and is highly conserved in species as diverse as yeast and man. In normal mammalian cells, the cdc2/p34 gene is induced by mitogenic growth factors and is expressed by all proliferating cells. By contrast, $\mathrm{cdc} 2 / \mathrm{p} 34$ expression is repressed when cells terminally differentiate or undergo senescence. Moreover, $\mathrm{cdc} 2 / \mathrm{p} 34$ regulation is deranged in some spontaneous and oncogene-transformed neoplastic cells.

We planned to clone the regulatory sequences of the human cdc2/p34 gene; to identify the sequences important in regulating the responses to mitogens, terminal differentiation, senescence, and oncogenic transformation; and to identify the cellular transactivator proteins that interact with these sequences to control expression.

\section{Accomplishments}

We have successfully cloned the entire human cdc2 gene, including substantial 3 ' and 5 ' flanking se- quences. We have shown that $1.7 \mathrm{~kb}$ of 5 ' flanking sequence are sufficient for cell cycle dependent expression. This result strongly suggests that the $\mathrm{cdc} 2 / \mathrm{p} 34$ regulatory sequences are contained within $1.7 \mathrm{~kb}$ of the 5 ' flanking region. We have determined the sequence of this entire region, as well as a substantial amount of the downstream region. The 5 ' flanking region contains several potential binding sites for regulatory transcription factors. The organization of this 5 ' flanking region and the potential transcription factor binding sites within it are shown by Figure 1 .

This sequence tells us several important things about how cdc2/p34 gene expression is controlled. First, it is likely to be stimulated by the c-fos and/or c-jun protooncogene (through sites labeled AP-1 on Figure 1). Second, it is likely to be negatively controlled in part by the retinoblastoma tumor suppressor protein (through the portion of the gene labeled RCE on Figure 1). This is an important finding because several lines of evidence suggest that this tumor suppressor protein is important for suppressing the growth of human cells through the process of cellular senescence. Third, the cdc2/p34 gene is likely to be controlled by a family of transcription factors designated bHLH factors (through sites labeled HLH, A and HLH, B in Figure 1). This is important because we have indirect evidence that bHLH factors are also very important in suppressing the growth of human cells, and we expect that one or more bHLH factor may be an as yet unidentified tumor suppressor for human cells.

A grant entitled " $\mathrm{Cdc} 2$ gene regulation in cell cycle and senescence" was submitted to the National Institutes of Health in October, 1992 as a result of this LDRD-funded project. A manuscript describing the organization and 5 ' sequence of the human cdc2/p34 gene is in preparation. 


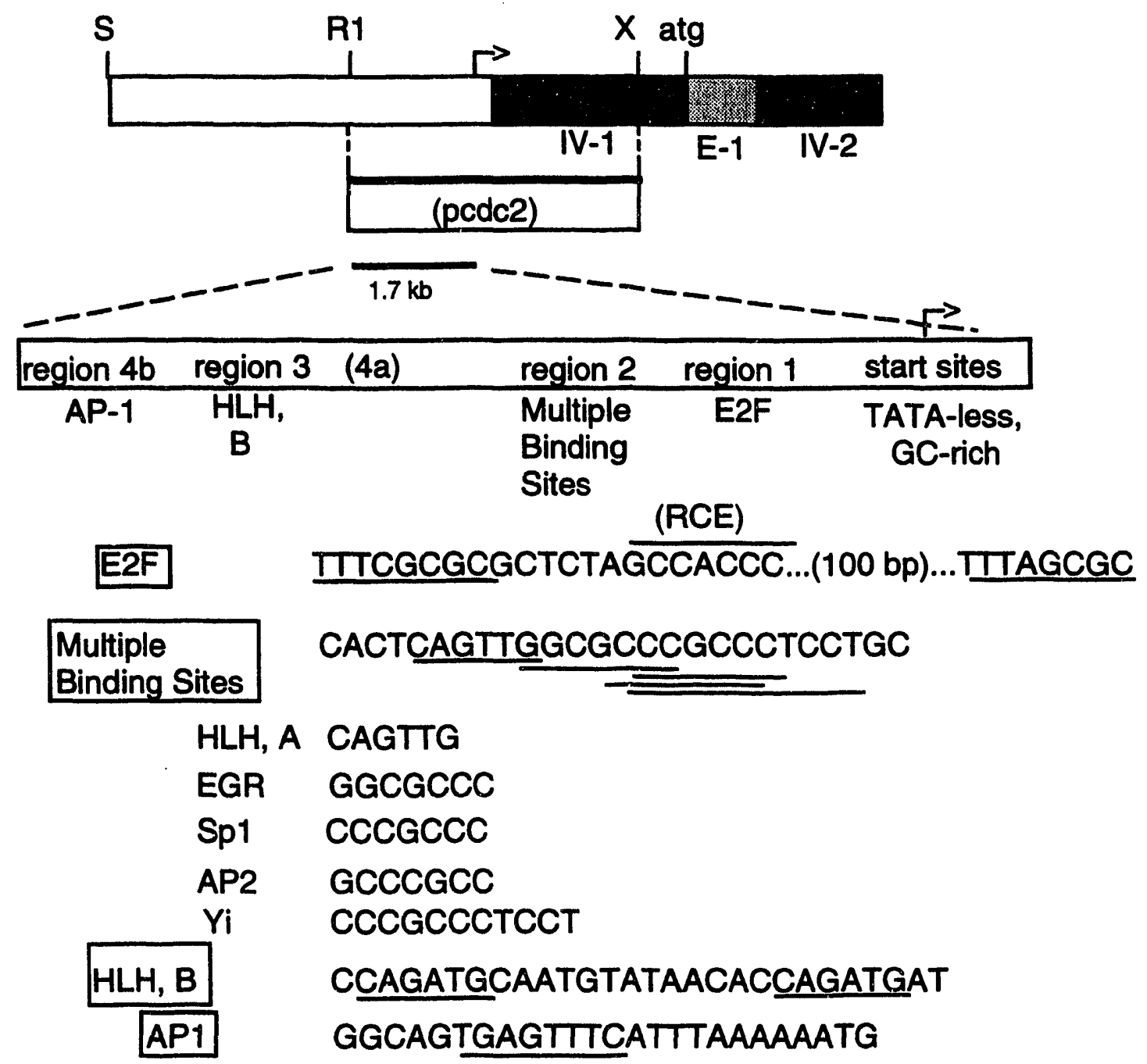

Fig. 1. Potential $5^{\prime}$ regulatory elements in the human cdc2 gene. $S=$ Sall; $R 1=E c o$ RI; $X=X h o l ;$ Hooked arrow $=$ start sites of transcription; IV = intervening sequence; $E=$ exon; atg = start site of translation; $E 2 F=E 2 F$ binding site; $R C E=$ retinoblastoma control element $H L H=$ helix-loop-helix protein binding site; $E G R=$ early growth response element; $S P 1=S P 1$ binding site; $A P-2, A P-1=A P$ 2 and $A P-1$-like binding sites; $Y i=Y i$ complex binding site. 


\section{Application of the Micropipette Tech- nique to the Measurement of Erythroid Viscoelastic Properties during Termi- nal Differentiation}

Principal Investigator: Joel Anne Chasis

Funding: $\$ 20,700$

\section{Project Description}

The overall aim of this project was to establish a technique for measuring mechanical properties of nucleated erythroid progenitor cells. Although the biophysical properties of the mature red cell have been characterized in detail, no measurements of mechanical properties in their immature precursors have been reported. We are interested in characterizing membrane properties of these progenitor cells because mechanical properties are regulated by membrane protein interactions and an understanding of membrane properties will therefore lead to an increased understanding of membrane organization and assembly during erythroid differentiation.

\section{Accomplishments}

For these studies we established a model system for erythroid differentiation utilizing mouse

erythroleukemia (MEL) cells. In the presence of DMSO, MEL cells were induced to undergo differentiation thus providing us with various time points for study. We used micropipette technique as a means of measuring mechanical properties since this methodology has been applied to several nucleated cell-types including granulocytes and lymphocytes. With time and temperature dependent experiments we first established that the optimum conditions for MEL cells were at $10^{\circ} \mathrm{C}$ so we created a cooling stage to maintain a constant $10^{\circ} \mathrm{C}$ temperature. We also developed a method of nuclear staining with Hoechst 33342 stain to enable visualization of the nucleus during micropipette aspiration. This allowed us to aspirate into the pipette only membrane and cytoplasm so as not to complicate the data with nuclear properties. Utilizing micropipettes of $\sim 2 \mu \mathrm{m}$ diameter we applied increasing suction pressure and measured the cell entry flow. Analysis of these data provides us with a measurement of the elastic shear modulus of the membrane. These studies are still ongoing and will be continued under a five-year grant from the National Institutes of Health.
Together these studies provide a biophysical framework for understanding membrane assembly during erythroid differentiation.

\section{Environmental Pollutants and Oxida- tive Stress}

Principal Investigators: Trudy Forte and Ronald Krauss

Funding: $\$ 90,500$

\section{Project Description}

The overall objectives of this research were (1) to study the effect of environmental pollutants such as ozone and other free radical generators on plasma lipoproteins and (2) to determine whether specific antioxidants can protect plasma components, particularly lipoproteins, from oxidative stress. Since it was technically more difficult to adequately expose plasma to ozone, we exposed plasma to the gas phase of cigarette smoke; the cigarettes used were KU 2R1 cigarettes that produce known quantities of NOx, which is also an atmospheric oxidant.

\section{Accomplishments}

We found that free radical generators in gas phase of smoke oxidatively modify lipoproteins when plasma is exposed to such agents. Increased electrophoretic migration was used as an indicator of lipoprotein oxidation, where it is known that oxidized lipoproteins have an increased negative charge. Oxidation, as determined by agarose electrophoresis, was most pronounced in low-density lipoproteins (LDL) followed by high-density lipoproteins (HDL). Figure 2 shows the percent increase in electrophoretic migration as a function of time of exposure to oxidant stress. Plasma antioxidant levels, including water soluble vitamin $C$ and uric acid and lipid soluble vitamin $E$ were also examined in parallel with oxidant exposure. We found that vitamin $C$ was completely depleted after 1-hr exposure to oxidants while vitamin E declined gradually and was $40 \%$ of control values after 6-hr incubation. Depletion of uric acid was intermediate to vitamin $C$ and $E$. We carried out experiments to determine whether exogenously added antioxidants could protect lipoproteins. The antioxidants tested included water soluble types, vitamin $C$ and glutathione, and lipid soluble vitamin E. Protection by 

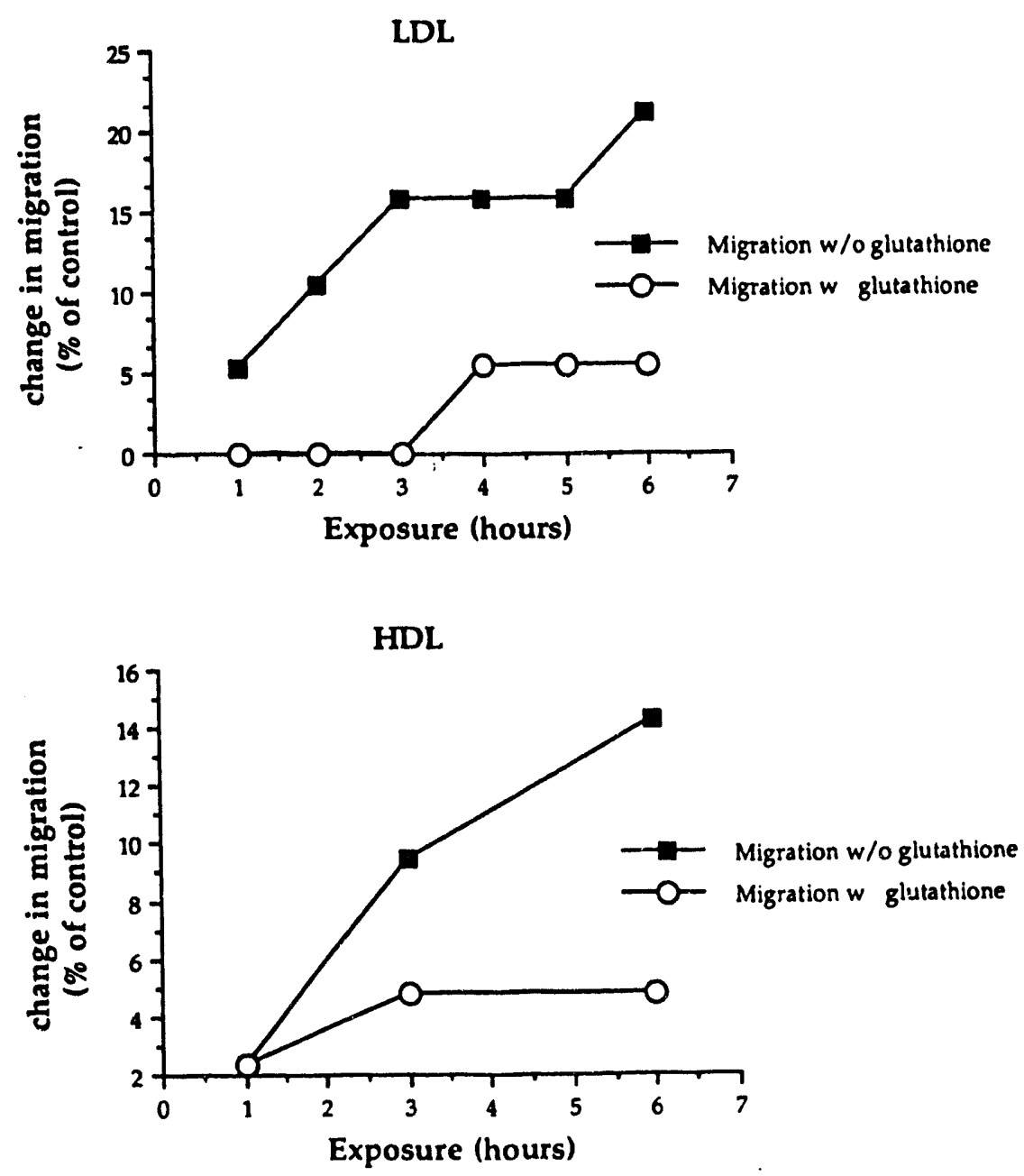

Fig. 2. Effect of oxidants in gas phase of cigarette smoke on the electrophoretic mobility of lowdensity lipoproteins ( $L D L)$ and high-density lipoproteins (HDL). Plasma was exposed to gas phase of cigarette smoke in the presence or absence of exogenous glutathione and sampled every hour for up to $6 \mathrm{hr}$ of exposure; increase in migration relative to control is indicated by closed symbols. The protective effect of $1 \mathrm{mM}$ glutathione added to the plasma is indicated by the open symbols.

vitamin $C$ and vitamin $E$ were modest; however, we found that $1 \mathrm{mM}$ glutathione substantially protected LDL and HDL from oxidation as shown in Figure 2.

The increased electrophoretic migration of LDL and HDL could be ascribed to changes in apolipoprotein (apo) structure since numerous laboratories using isolated LDL exposed to copper ions have shown that apoB on LDL is fragmented and subsequently such LDL have an increased negative charge. Following exposure of plasma to oxidative stress we isolated both LDL and HDL to determine whether their apolipoprotein structure was altered. The results were surprising since (1) the LDL apoB showed no degradation. This suggests that oxidation produced more subtle changes that resulted perhaps in changes in conformation of the apoB protein, which in turn exposes more negatively charged amino acids. (2) HDL proteins showed major changes that were manifested by the appearance of additional protein bands that were larger in size than the expected HDL proteins. Immunoblots for specific HDL proteins including apoAI and apoAII showed that the additional bands were polymeric forms of these two proteins; some bands were homodimers or trimers of apoAI while others were heterodimers and trimers containing apoAI and apoAII. These results indicate that apolipoproteins on HDL are crosslinked during oxidation. Such crosslinking could affect the metabolism of lipoproteins, particularly its ability to remove 
excess cholesterol from cells. A defect in cholesterol removal would result in increased risk of atherosclerosis.

The most significant finding in our investigations was that the enzyme, lecithin:cholesterol acyltransferase (LCAT), which is necessary for the normal metabolism of $\mathrm{HDL}$, was an exquisitely sensitive marker for oxidative damage in plasma. This enzyme, which catalyzes the esterification of free cholesterol to cholesteryl ester in HDL, exhibited a $60 \%$ decrease in activity in the first hour of exposure to oxidative stress, see Figure 3. Decreased LCAT activity in man is associated with increased risk to atherosclerosis, thus our findings have important health related implications.

The time frame of disappearance of LCAT activity and depletion of plasma vitamin $C$ were similar. Thus we examined the possibility that exogenously added vitamins could protect plasma constituents, particularly LCAT, from oxidative damage. We examined both the water soluble vitamins, vitamin $C$ and glutathione, and the lipid soluble vitamin $\mathrm{E}$ for their ability to protect against oxidation. Vitamin $C$ and vitamin $\mathrm{E}$ had only a very small protective effect on LCAT. The most exciting finding was that the thiol protein antioxidant, glutathione, was a good protector of LCAT activity (Figure 3 ) and at the same time appeared to protect HDL proteins from crosslinking.

LCAT ACTIVITY

Oxidant Exposure

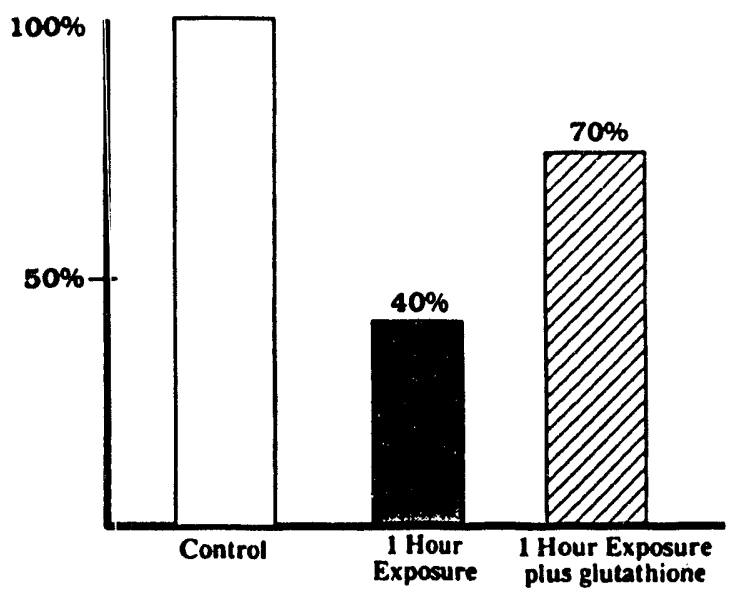

Fig. 3. Effect of oxidants on lecithin:cholesterol acyltransferase (LCAT) activity. Oxidants rapidly decrease LCAT activity in the first hour of exposure. Addition of $1 \mathrm{mM}$ glutathione to the plasma provides substantial protection of LCAT activity during the first hour of exposure.
The results of our study indicate that our test system, which uses whole plasma, appears to be a very sensitive system for studying both oxidative events and mechanisms for reducing oxidative damage in lipoproteins and plasma enzymes involved in lipoprotein metabolism.

\section{Publications}

M. McCall, F. Kuypers, J. van den Berg, D. Tribble, L. Knoff, and T. Forte, "Cigarette Smoke Oxidants Modify Plasma Lipoproteins and Impair LCAT Activity," Am. Heart Assn. Arteriosclerosis Council, 66th Scientific Sessions (1992), p. 133.

M. R. McCall, F. Kuyper, R. M. Krauss, D. Tribble, L. Knoff, and T. M. Forte, "Oxidants in Gas Phase of Cigarette Smoke Modify Plasma Lipoproteins and Impair LCAT Activity," in preparation.

\section{Baseline Data for Soft X-ray Microscopy}

Principal Investigator: Robert Glaeser

Funding: $\$ 40,000$

\section{Project Description}

This project explores the promise of soft $x$-ray microscopy (SXM) to visualize features at the subcellular level, in a fashion that complements what can already be done by light microscopy and electron microscopy.

There are two objectives behind the design of these "base-line data" experiments. First of all, we will investigate whether soft $x$-ray imaging can provide new or better types of image contrast, even if the nature of the specimen preparation is otherwise "conventional," for example, fixed specimens, critical point, and whole-mount preparations. Secondly, we will determine whether there are some types of morphological studies that can be done in water, without fixation, dehydration, and staining, in which the resolution would well exceed that of the light microscope and in which the damage to the macromolecular and subcellular structure would remain well below the resolution of the soft $x$-ray image.

\section{Accomplishments}

Project funding has enabled the appointment of a fulltime electron microscope technician who has prepared 
test specimens (honey bee flight muscle and eye) for thin sectioning. Work has begun to study the use of lanthanum, terbium, and europium salts/chelates as radiation-hard fluores. Once confirmed, these fluores could then be coupled to biological probes and used to map genes of interest by a technique known as chromosome painting, or to visualize the spatial arrangement of different types of filaments in the cytoskeleton. Additionally, arrangements have been made to secure thin-section samples that have already been used to generate images of the spatial variation of calcium within organelle-compartments of the cell by other investigators, using electron probe techniques. As these samples are already well-characterized by electron-beam microanalysis, we will evaluate whether SXM compares favorably in monitoring the changes in ionic concentration during enzymatic actions, such as muscle excitation. All of our preliminary work is being done with dry (fixed, embedded) sample materials because of the known effects that very high levels of radiation have on wet, living specimens.

\section{A Comprehensive Approach to Protein Folding}

Principal Investigator: Teresa Head-Gordon

Funding: $\$ 10,800$

\section{Project Description}

The protein folding problem is widely acknowledged to be one of the most intriguing challenges in molecular biophysics. The benefits derived from its solution include the reengineering of defective proteins, the design of synthetic proteins, and the understanding of how molecules self-assemble. Basic research that is comprehensive in its approach to protein folding would involve an understanding of (1) the folding kinetics, (2) the prediction of tertiary structure from the amino acid sequence alone, and (3) the quantitative description of the conformational properties of solvated peptides. The successful development of these three areas will put the necessary science in place in order to fully develop the potential of the sequence database anticipated from the Human Genome project.

While the long-term vision of this work is to provide a comprehensive theoretical approach to structure prediction and molecular self-assembly for proteins in vivo, we focused on the following objectives in the short term:
- A qualitative understanding of the relative roles of the specifics of the amino acid sequence and solvent environment in the initial stages of protein folding.

- The development of semi-empirical algorithms that reliably predict tertiary structure from the amino acid sequence.

- A first step toward quantitative modeling of small solvated peptides. Again we emphasize that for genuine predictive studies of protein folding, methods that are quantitatively reliable must be developed.

\section{Accomplishments}

First statistics are being run on the effect of full hydrogen bonding on the hydrophobic interaction of two methanes in water. In addition to 2 IBM workstations, these calculations are also running on a Cray at AT\&T Bell laboratories. These calculations are computationally intensive, and we have recently coded a force bias Monte Carlo sampling scheme to speed up their convergence. We expect results by January 1993.

Secondly, we have found a number of important insights into neural network design that should improve prediction of real protein structure. This work has now laid sufficient groundwork for the antlion method so that a strong grant proposal to AFOSR will be submitted by January 1993. A manuscript on neural network predictions of protein structure has been submitted for publication in Physical Review A.

Finally, electronic structure software has been ordered that should allow beginning the calculations on solvent environment on the conformations of small peptides.

\section{Publications}

F.H. Stillinger and T. Head-Gordon, "A View of Inherent Structure of Water Using an Orientational Perturbation Theory," submitted to Phys. Rev. A. (1992).

T. Head-Gordon and F.H. Stillinger, "An Orientational Perturbation Theory for Pure Water," J. Chem. Phys., in press.

T. Head-Gordon and F.H. Stillinger, "Toward Optimal Neural Networks in Protein Structure Prediction," submitted to Phys. Rev. A. (1992).

F. H. Stillinger, T. Head-Gordon, and C. Herschfeld, "A Toy Model for Protein Folding," submitted to Phys. Rev. A. (1992). 


\section{Single DNA Molecule Chemistry and Manipulation for Gene Separation and Amplification: A New Sequencing Technique Using Organorhodium and Ruthenium Complexes}

Principal Investigators: Marcos F. Maestre and Richard H. Fish

Funding: $\$ 87,400$

\section{Project Description}

New and innovative techniques are urgently needed in order to map and sequence the human genome, at present an extremely laborious and expensive undertaking.

We used a novel approach to this important problem, which utilizes organometallic-sequence-specific oligonucleotide complexes where a portion of the sequence-specific oligonucleotide of this organometallic complex recognizes complemeritary sequences in single DNA molecules and, as well, the organometallic metal center, bound to another portion of the sequence-specific oligonucleotide, acts as an anchor for the organometallic- sequence-specific oligonucleotideDNA complex to the glass and electrode surface of the microelectrode stage of an epi-fluorescence microscope. The anchored single DNA molecule is visualized with ethidium bromide as the fluorescence label, and then selectively cut by optical or mechanical methods, and manipulated on the microelectrode stage of the epi-fluorescence microscope; this technique, together with PCR amplification, for physical mapping and sequencing single DNA molecules.

\section{Accomplishments}

To date many experiments have been carried out showing that it is indeed possible to visualize individual molecules of DNA beiween two cover slips on the stage of a fluorescence microscope. The dynamics and motion of each of these molecules have been followed, and the various global transitions involving the processes of super-coiling condensation, collapsing, and simple coiling have been recorded. The molecules studied so far are: (a) Polydispersed calfthymus DNA; (b) lambda DNA restriction fragments; (c) T-4 DNA; and (d) cosmid DNA. Various fluorescent ligands have also been used, such as Acridine orange, DAPI, and Hoechst 33258. In addition, preliminary experiments have shown that it is possible to use the microscope to flow single DNA molecules of DNA migrating in a thin micro-gel under an applied electric field. Several interesting observations have also been made about the trapping of molecules by the gel matrix.

New data from this project include the bonding studies of the ( $h^{5}$-pentamethylcyclopentadienyl) rhodium (aqua) complex to nucleobases, nucleosides, nucleotides, and sequence-specific oligonucleotides.

Extensive new bonding studies were conducted to clearly show selective $C \mathrm{p}^{*} \mathrm{Rh}$ bonding to the $12-\mathrm{A}$ portion of the 24-mer. We have also made considerable progress on the application of anchoring 1-DNA to the glass and electrode surface of an epi-fluorescence microscope stage with a clear indication that the 1DNA is anchored, via the Cp*Rh-24-mer complex, on the terminal end to the glass and microelectrode surfaces. These experiments have been carried out with a fluorescence microscope equipped with a super-intensified ultra-low light-level camera. The data are stored in a video tape form and are amenable to further digitization and processing. The processing and analysis of the images of DNA have provided much new information on the interaction of DNA with the electric field and the gel through which it is traveling.

\section{Publications}

L. Song and M.F. Maestre, "Observation of DNA Molecules Undergoing Capillary Electrophoresis," J. Biomolecular Structure and Dynamics 9, 525 (1991).

L. Song, and M.F. Maestre, "Unhooking Dynamics of U-Shaped DNA Molecule Undergoing Gel Electrophoresis," J. Biomolecular Structure and Dymamics 9, N1, 87 (1991).

D.P. Smith, E. Baralt, B. Morales, M.M. Olmstead, M.F. Maestre, and R.H. Fish, "Bioorganometallic Chemistry. 1. Synthetic and Structural Studies in the Reactions of a Nucleobase and Several Nucleosides with a $\left(\mathrm{h}^{5}\right.$ Pentamethylcyclopentadienyl)rhodium Aqua Complex," J. Am. Chem. Soc. (1992), in press.

D.P. Smith, M.M. Olmstead, M.F. Maestre, and R.H. Fish, "Bioorganometallic Chemistry. 2. A Synthesis and Structural Study of the Reaction of the Nucleobase, 1-Methylcytosine, with a $\left(\mathrm{h}^{5}\right.$ Pentamethylcyclopentadienyl)rhodium Aqua Complex," submitted to Organometallics . 


\section{Experimental Testing of Novel Mis- match Repair Enzymes for Mapping Natural Genetic Polymorphisms}

Principal Investigators: Elaine Ostrander and Jasper Rine

Funding: $\$ 100,000$

\section{Project Description}

Our primary interest is in mapping genes that regulate mammalian behavior. The anecdotal observation that some mammalian behaviors are instinctive, and therefore controlled at the genetic level, was made centuries ago. Until recently, however, advances in molecular biology and neurobiology have been insufficient to identify genes that control behavior. At the heart of the problem is the fact that confusing patterns of inheritance are typically seen in animal breeding studies, suggesting that most behaviors are complex; they reflect the additive effects of many genes that are spread throughout the genome and therefore inherited independently.

We tested the hypothesis that Quantitative Trait Loci (QTL) analysis can be used for identifying genetic loci responsible for inherited behavior patterns in mammals. This experiment requires that observations be made in a mammal for which groups or subspecies of individuals display very different behavior patterns. Within each group, however, it is necessary for purposes of quantitation that unrelated individuals display a high degree of behavioral uniformity. We have chosen, therefore, to make our observations in the domestic dog. Well-known canine behaviors that are breed-associated, and hence presumably controlled at the genetic level, include herding by Sheep Dogs, the stance of Pointers, retrieval by Goldens, and tracking by Bloodhounds. We are particularly interested in Border Collies that are well-known for their instinctive herding behaviors and Newfoundlands that are recognized for their remarkable water rescue skills.

As a first step toward identifying genes responsible for instinctive behavior patterns in dogs, we faced the task of constructing a genetic map of the canine genome. Studies with the human genome have shown that the most useful type of studies from which to conduct a genetic map are those which are highly polymorphic in populations, but relatively stable within pedigrees. Markers should be easy to use and data easily transmitted between laboratories. We chose to base our map on (CA) $n$ repeats, a class of simple sequence repeats (SSR) that occur with high frequency in mammalian genomes. A (CA)n locus is generally comprised a run of 10-30 units that show polymorphism in the number of times the repeat is reiterated. Alleles are typically measured by a PCR-based assay in which primers made from unique sequences that bracket the repeat are used to measure the length of (CA)n alleles.

\section{Accomplishments}

A. Development of a New Strategy for Collecting Large Numbers of $(C A)_{n}$ Repeats. Our initial task was to collect a large number of (CA)n repeats. Together with our collaborator Geoff Duyk we developed a method to prepare "marker selected libraries" in which 40$50 \%$ of the inserts contain [CA]n repeats, and hence, potentially useful polymorphisms. In this approach a dut-ung strain of E.coli is used for preparing a pool of single-stranded DNA from a total canine genomic library. In the next step, a primer extension reaction is done using a (CA) 15 oligonucleotide. Products of the primer extension reactions are then transformed back into a wild type E.coli strain. Primarily those clones that have a (CA)n repeat become double stranded in the primer extension reaction, and hence, are maintained following transformation back into wild type E.coli. Because of the efficiency of this approach, enough markers were collected to make a genetic map of the entire canine genome (5-10 cM resolution), in a very short time. This strategy has now been adapted by several labs working in human genetics and has greatly reduced the workload necessary for collecting large numbers of markers. To date we have sequenced about 150 of these markers using an ALF automated sequencer and characterized over 60 in terms of frequency and PIC values. Our results suggest that microsatellites will be as useful for genetic studies in dogs as they have been in humans. We are continuing to sequence and characterize new markers weekly.

B. Adaptation of Automated Sequencer for Genetic Mapping. The time required for this effort has been dramatically reduced by our success at adapting an automated sequencer for measuring alleles as required for genetic mapping studies. Previously, assays were conducted by labeling one of two oligonucleotides/ pair with 32-P and then assaying radioactive $P C R$ products on standard denaturing sequencing gels. We have optimized a strategy for tagging PCR primers with fluorescine and analyzing PCR reactions on an automated sequencer. Joe Katz from the LBL engineering group has collaborated with us to develop a computer program called FRAGMAN, which converts data from the graphical format provided by the ALF to a useful numerical format. FRAGMAN determines 
sizes of $P C R$ products using internal standards. The FRAGMAN program is currently being optimized for distribution.

C. Construction of Canine/hamster Cell Lines. To facilitate the ordering of these markers into a useful genetic map we are taking a number of approaches. Among the most productive has been a collaboration with Kelly Frazer and David Cox that resulted in the production of hamster/canine fusions. 200 such lines have been produced and 50 are currently being characterized. A dog specific repetitive element identified by G.L. Stiegler and colleagues in Richland, Washington is being used to screen the cell lines. Once the dog chromosomes in these lines are fully characterized, this reagent may be used to facilitate physical as well as genetic mapping studies. Constructing sublines of radiation hybrids from these fusions should make the task of assigning STSs to particular locals straightforward. A particular effort will be made to construct a radiation hybrid map of the dog $X$ chromosome, which is the subject of intense study by several groups.

D. Conseroation Biology. One particularly pleasing outgrowth of this work has been the interest shown by people working in the fields of evolutionary biology. Early results from collaborative studies between myself and Robert Wayne (UCLA and the London Zoological Institute, London Zoo) have shown that markers isolated for genetic mapping in the dog work extremely well with other Canidae, such as wolves and foxes. This result was a pleasant surprise since there has been limited success transmitting markers isolated from one animal species to another. The expectation is that surh markers may be used to aid in breeding decisions by zoos and biologists struggling to maintain diversity in endangered species.

E. Other Collaborations. Since humans and dogs share many disorders, we are interested in collaborating with other groups who are mapping genes responsible for various canine disorders. Of particular interest are those disorders that are usually spontaneous in humans but familial in dogs. Understanding the genetic basis of these types of diseases will provide information about the pathology of the disease, as well as genetically predisposing factors. We have, therefore, initiated collaborations with groups trying to map genetic basis of several canine disorders including congenital neutropenia, congenital heart disease, and familial glomerulonephritis.

\section{Publications}

E.A. Ostrander, G.F. Sprague, and J. Rine, "Identification and Characterization of Simple Sequence Repeat Markers for Genetic Mapping in Dog," in preparation.

R.K. Wayne and E.A. Ostrander, "Molecular Evolution of the Dog Fanily," in preparation.

E.A. Ostrander, P.M. Jong, J. Rine, and G. Duyk, "Construction of Small Insert Genomic DNA Libraries which are Highly Enriched for Microsatellite Repeat Sequences," Proc. Natl. Acad. Sci. USA 89, 3419 (1992).

E.A. Ostrander and J. Rine, "Mapping the Genetic Basis of Morphology and Behavior in the Dog," in The Genetics and Molecular Biology of Complex Diseases Banbury Report, Eds. E. Lander, A. Chakravarti, and J. Witkowski, Cold Spring Harbor Press, Cold Spring Harbor, New York (in press).

E.A. Ostrander, P. Jong, P. Mapa, G. Sprague, and J. Rine, "Construction of a Genetic Map of the Dog Genome for Mapping Complex Behavioral and Morphological Traits," presented at the 1992 Cold Spring Harbor Genome Mapping and Sequencing Meeting.

\section{Maintenance of Phenotypic Traits in Cultured Human Mammary Epithelial Cells}

Principal Investigator: Paul Yaswen

Funding: $\$ 20,700$

\section{Project Description}

In order to make mechanistic associations and to estimate probabilities associated with human carcinogenesis, in vitro models that are easily manipulated are required. Major advances have been made in recent years in the ability to culture cells isclated from a variety of human organs. However, most long-term tissue culture systems employed to date are not able to support the full range of phenotypes exhibited by the organ of origin. Artificial constraints placed on cultured cells often lead to the outgrowth of particular cell populations and loss of regulated functions. The shortcomings of available systems for studies of 
carcinogenesis are manifested in two ways: (1) The cell types that are most susceptible to carcinogenic insuits in vivo may not be available in vitro and (2) the parameters that define carcinogenic progression in vivo may not be applicable in vitro. These problems are particularly acute in studies of human breast carcinogenesis. Under ordinary culture conditions, where breast epithelial cells are grown as monolayers on impermeable supports in the absence of basement membrane material, detection of early malignant changes such as loss of the capacity to maintain cellular polarity and vectorial secretion is not possible. Furthermore, susceptibility of epithelial cells to malignant transformation may be directly influenced by the cifferentiated state and the extracellular microenvironment or the target cells. For example, the cells composing most infiltrating breast ductal carcinomas display the differentiation marker, keratin 19. Normal epithelial cells of the luminal lineage, displaying this marker, are not observed under current culture conditions. Consequently, part of the difficulty observed in transformation of cultured human epithelial cells may be due to the inability of current in vitro systems to support cells with this phenotype. The objective of this project has been to improve upon the human mammary epithelial cell (HMEC) culture system so that it supports a wider range of differentiated phenotypes and functions.

\section{Accomplishments}

In order to develop a normal HMEC culture system that inore closely approximates the phenotypic diversity found in vivo, we have begun to examine both soluble and insoluble factors that influence certain parameters of differentiated breast cell function. In one set of experiments, the effects of calcium concentration and the presence or absence of fetal bovine serum in initial culture medium 'were compared for primary and secondary cultures derived from normal human breast tissue. Phenotypic assays have included microscopic analysis of cellular orientation and contacts, as well as immunohistochemical staining of keratins 14,18, and 19, polymorphic epithelial mucins, fibronectin, collagen IV, and laminin. These experiments indicated that the two variables had pronounced influence upon cell growth, $\mathrm{m} n$ tphology, and phenotypic expression in resulting cultures. Optimization of culture conditions using knowledge gained from these experiments is continuing. 


\section{Materials Sciences Division}

Resist Modeling for X-Ray Lithography

Principal Investigators: David Attwood, Andrew Neureather, and William Oldham

Funding: $\$ 13,400$ (FY92)

$\$ 97,900$ (FY91)

\section{Project Description}

Pattern transfer for microelectronic devices is presently accomplished through the use of visible light, imaging-forming optics, and appropriate recording materials. Movements toward the use of shorter wavelength radiation at ultraviolet (UV) and deep ultraviolet (DUV) wavelengths is currently underway. Further down the road is the possibility of using extreme ultraviolet or soft $x$-ray wavelengths (collectively XUV) that would eventually permit the manufacture of computer ships with increased capacities based on smaller feature sizes and increased numbers of components and capacity. One aspect of such a capability would need to be an ability to record detailed large field images at XUV wavelengths. The purpose of this project was to modify existing computer programs to permit their use in modeling resist exposures to these XUV waveleigths.

\section{Accomplishments}

In a collaboration between LBL's Center for X-Ray Optics and UC Berkeley's Department of Electrical Engineering and Computer Science, existing computer codes for modeling electromagnetic propagation, pattern transfer, and recording were modified to permit their use at very short XUV wavelengths and to attempt to access their veracity in this new spectral regime. Several graduate $s^{\wedge} u d e n t s$ were jointly emp.oyed in this task. The! programs were modified to incluale refractive index data for XUV wavelengths, particularly in the $130-\AA$ to $140-\AA$ region for relevant materials. Test runs were made for various models, including so-called reflective masks, the effect of defects in these masks, and phase effects due to propagation in the vicinity of various mask structures in addition to discussions about eventual testing of these models in experiments at XUV wavelengths.
Modeling of the resist properties was also included. Discussions were also held regarding the role of coherence properties of the radiation on pattern transfer capabilities and limitations thereof.

\section{MOCVD Growth and Characteriza- tion of II-VI Compounds Using Non- toxic Single Molecule Precursors}

Principal Investigators: Edith D. Bourret and John Arnold

Funding: $\$ 73,100$

\section{Project Description}

We are trying an innovative multidisciplinary approach to Metal-Organic Chemical Vapor Deposition (MOCVD) of II-VI compounds. The innovative approach is to use single-molecule precursors in which the metallic elements and the chalcogens are contained in a single molecule that presents many advantages, in particular low toxicity. Conventionally, the growth of II-VI compounds by MOCVD involves co-pyrolysis of metal alkyls (such as dimethylzinc for zinc compounds) with a volatile dialkyl or hydride chalcogenides (such as $\mathrm{H}_{2} \mathrm{Se}$ for selenides). The metal alkyls are toxic and pyrophoric, and the hydrides gases are highly toxic. Successful replacement of these conventional sources will have a major impact in the semiconductor industry.

The initial focus is on synthesis of the new precursors and on testing the suitability for use in the MOCVD process.

\section{Accomplishments}

Relatively few metal tellurolates and selenolates are known at present. The first step of the research is to better define the coordination chemistry of tellurolate (of general formula: $\mathrm{L}_{n} \mathrm{M}-\mathrm{Te} \mathrm{R}_{m}$ ) and analog selenolate ligands and, in doing so, to provide a rational basis for the design of molecular precursors. The second step is to demonstrate that the new precursors can be used for epitaxial growth of thin films. 
We have been focusing the work on the synthesis and structure determination of low-coordinate homoleptic zinc, cadmium, and mercury sitel and trisel derivatives (whose physical and chemical properties differ considerably from previously reported group 12(II) tellurolates) and testing the suitability for MOCVD growth of thin films. We found that previously encountered difficulties in preparing and handling toxic organotellurium compounds can indeed be avoided by use of more tractable higher molecular weight alkyl-, aryl-, and silyl derivatives. These compounds can be manipulated using conventional inert atmosphere techniques in an open laboratory, yet they are volatile enough to give intense molecular ions in their electron impact mass spectra.

This initial success led us to investigate related tellurolate and selenolate compounds. The synthetic utility of our tellurolysis route has been extended to prepare tellurium containing compounds with a wide range of main group, transition, and lanthanides elements. Specific examples of homoleptic (i.e., binary) compounds are as follows: (a) $\mathrm{M}$ (Sitel) with $\mathrm{M}=\mathrm{H}, \mathrm{Li}, \mathrm{Na}, \mathrm{K}$; (b) $\mathrm{M}(\mathrm{Sitel})_{2}$ with $\mathrm{M}=\mathrm{Mg}, \mathrm{Ca}, \mathrm{Sr}, \mathrm{Ba}$, $\mathrm{Mn}, \mathrm{Co}, \mathrm{Fe}, \mathrm{Zn}, \mathrm{Cd}, \mathrm{Hg}, \mathrm{Sn}, \mathrm{Pb}, \mathrm{Eu}$; (c) M(Sitel) 3 with $\mathrm{M}=\mathrm{Sc}, \mathrm{Y}, \mathrm{La}, \mathrm{Ce}, \mathrm{Sm}, \mathrm{Eu}, \mathrm{Ga}$, In; and (d) M(Sitel) ${ }_{4}$ with $\mathrm{M}=\mathrm{Ti}, \mathrm{Zr}, \mathrm{Hf}$.

Our selenolate chemistry was started more recently, but initial results are quite encouraging and demonstrate that our new synthetic route is widely applicable and lead to the synthesis of important new classes of precursors previously inaccessible due to the limitations of the conventional metathesis type of approach.

We have determined the crystal structure of two zinc tellurolates and also the first monomeric cadmium tellurolate. These compounds have been investigated in solution using ${ }^{1} \mathrm{H}$ and ${ }^{125} \mathrm{Te}$ NMR spectroscopy and in the gas phase by electron-impact mass spectroscopy. For all three metals, we observe only the monomeric compound in the gas phase. In solution, we have discovered strong evidence for the existence of dimeric $\left[\mathrm{M}(\mathrm{Sitel})_{2}\right]_{2}$ species. For the zinc complex, further evidence for the latter structure was obtained from single crystal $x$-ray diffraction.

Several compounds have been tested for their suitability as precursors in the MOCVD process. Clean pyrolysis reactions and deposition of thin films was achieved from $\left\{\mathrm{Zn}\left[\mathrm{Te}-\mathrm{Si}-\left(\mathrm{Si}_{-}-\mathrm{Me}_{3}\right)_{3}\right]_{2}\right\}_{2}$ and the $\mathrm{Cd}, \mathrm{Hg}$ analogs as well as from $\left[\mathrm{Zn}\left[\mathrm{Se}-\mathrm{C}-\left(\mathrm{Si}_{-} \mathrm{Me}_{3}\right)_{3}\right]_{2}\right\}_{2}$. Deposition of $\mathrm{ZnTe}$ films has been obtained in the temperature range of 250 to $350^{\circ} \mathrm{C}$ and the CdTe films in the temperature range of 280 to $350^{\circ} \mathrm{C}$ at atmospheric pressure and reduced pressure with nitrogen as the carrier gas. ZnSe films have been deposited at low pressure in the temperature range of 300 to $350^{\circ} \mathrm{C}$. Particle induced $x$-ray emission (PIXE) spectroscopy indicates that the films are stoichiometric and their crystalline structure was revealed by $x$-ray diffraction.

\section{Publications}

J. Arnold, J.M. Walker, K.M. Yu, P.J. Bonasia, A.L. Seligson, and E.D. Bourret, "Growth of II-VI Thin Films from Single-Source Precursors Based on Sterically Encumbered Sitel Ligands," Journal of Crystal Growth (1992), in press.

P. Bonasia, A. Seligson, J. Arnold, E. Bourret, and J. Walker, "Volatile Homoleptic Tellurolates and Selenolates of $\mathrm{Zn}, \mathrm{Cd}$ and $\mathrm{Hg}$ and Their Use as Singlesource Precursors to II-VI Semiconducting Thin Films," presented at the 203rd National Meeting, American Chemical Society, San Francisco, April 5-10, 1992.

J. Arnold, J.M. Walker, K.M. Yu, P.J. Bonasia, A.L. Seligson, and E.D. Bourret, "Growth of II-VI Thin Films from Single-Source Precursors Based on Sterically Encumbered Sitel Ligands," presented at the 6 th International Conference on Metalorganic Vapor Phase Epitaxy, Cambridge, MA, June 8-11, 1992.

A.L. Seligson, P.J. Bonasia, J. Arnold, K.-M. Yu, J.M. Walker, and E.D. Bourret, "Single Source Precursors for the Growth of Metal-Chalcogenide Thin Films," presented at the MRS Fall 92 Meeting, Nov. 30-Dec. 4, 1992, Boston, MA.

\section{Optical Investigations of Quantum Well Structures under High Magnetic Fields}

Principal Investigator: Daniel S. Chemla

Funding: $\$ 426,100$ (FY92) $\$ 346,100$ (FY91)

\section{Project Description}

This project was for the investigation of the optical properties and the dynamics of elementary excitations of gallium arsenide quantum wells under very high magnetic fields.

Modern crystal growth techniques are now able to produce very high-quality semiconductor thin films 
with thickness controlled at atomic-layer level. These novel artificial materials have had a tremendous impact on the fundamental sciences of solid state and have already produced numerous applications. Attempts to produce lower dimensionality nanostructures (quasi-1D or 2D) have been plagued by the difficulties of fabricating samples without defects and of avoiding size fluctuations. An elegant way to avoid these'difficulties and yet investigate the physics of low dimensionality is to apply high magnetic fields to semiconductor thin films to further the confinement to $O D$ and $1 D$.

We exploited the recent advances in time-resolved laser spectroscopy and the availability of split-coil superconducting magnets to investigate the physical properties of elementary excitation in low dimensionality regime. By applying large magnetic fields perpendicular or parallel to the plane of a high-quality quantum well structure, the electron hole pairs are further confined to quasi-OD and quasi-1D.

\section{Accomplishments}

The optical laboratory has been completed. New timeresolved detection system have been developed and tested. An advanced data-acquisition system has been designed and constructed. It was tested by performing femtosecond-time resolved nonlinear spectroscopy experiments on GaAs/AlGaAs quantum well structures without magnetic field applied.

The high field split-coil magnet had been delivered during the summer. It was tested, magnetic field up to 12 Tesla were obtained at temperature of the sample holder of $4 \mathrm{~K}$.

Experiments were pursued at AT\&T Bell laboratories using a conventional magnet with only one window. We demonstrated for the first time that the reduced density of states modifies completely the relaxation of carriers by limiting the number of available scattering channels. We also observed for the first time that magnetic confinement reduces exciton-exciton interaction and that this later depends strongly on the angular momentum. Relaxation by spin reversal of excitons optically active states to "dark" states (inactive in one-photon transitions) was discovered.

\section{Publications}

J.B. Stark, W.H. Knox, and D.S. Chemla, "Femtosecond Circular Dichroism Study on Non-thermal Carrier Distributions in Two and Zero Dimensional Semiconductors," Phys. Rev. Lett. 68, 3080 (1992).
T.B. Stark, W.H. Knox, and D.S. Chemla, "Spin Resolyed Femtosecond Magnetoexciton Interaction in GaAs Quantum Wells," to be published in Phys. Reo. B.

\section{High-T $T_{c}$ Superconducting Magnetometer}

Principal Investigator: John Clarke

Funding: $\$ 56,300$

\section{Project Description}

This project is aimed at the development of more reliable, lower noise dc SQUIDs (Superconducting QUantum Interference Devices) with a magnetic field resolution at $77 \mathrm{~K}$ sufficient for geophysical applications. To achieve this goal, it is necessary to understand the mechanism for the $1 / f$ noise at low frequencies $(f)$ and to implement procedures to reduce it.

The SQUIDs were made by laser-depositing $\mathrm{YBa}_{2} \mathrm{Cu}_{3} \mathrm{O}_{7-\mathrm{x}}(\mathrm{YBCO})$ films on $\mathrm{SrTiO}_{3}$ bicrystal substrates with a misorientation angle of $24^{\circ}$. The YBCO forms a grain boundary along the boundary of the bicrystal. Twelve dc SQUIDs were photolithographically patterned and acid etched so that two junctions were formed in each at the grain boundary. The SQUIDs were cooled in liquid nitrogen contained in a dewar surrounded by a triple mu-metal shield to reduce static and fluctuating magnetic fields. The electrical properties of each SQUID were measured in turn.

\section{Accomplishments}

The current-voltage (I-V) characteristics of the SQUIDs were close to those of the resistively shunted junction model. Figure 1 shows the total critical current $\left(2 \mathrm{I}_{\mathrm{c}}\right)$ and asymptotic resistances $\left(R_{N} / 2\right)$ for each of the twelve SQUIDs on a single chip. The average values are $2 \mathrm{I}_{\mathrm{c}}=185 \mu \mathrm{A}$ and $\mathrm{R}_{\mathrm{N}}=0.78 \Omega$, with standard deviations of $\pm 30 \%$ and $\pm 20 \%$, respectively. Notice that high values of $2 \mathrm{I}_{\mathrm{C}}$ tend to be correlated with low values of $R_{N} / 2$ and vice versa, suggesting that part of the scatter in the parameters arises from variations in the widths of the junctions; indeed the standard deviation in $\mathrm{I}_{\mathrm{C}} \mathrm{R}_{\mathrm{N}}$ is lower, about $\pm 18 \%$. Figure 1 also shows the maximum voltage signal $(\Delta \mathrm{V})$ available from 9 Type A SQUIDs and 3 Type B SQUIDs with estimated inductances of $150 \mathrm{pH}$ and $40 \mathrm{pH}$, respectively. The scatter in $\Delta \mathrm{V}$ is about $\pm 20 \%$. The mea- 


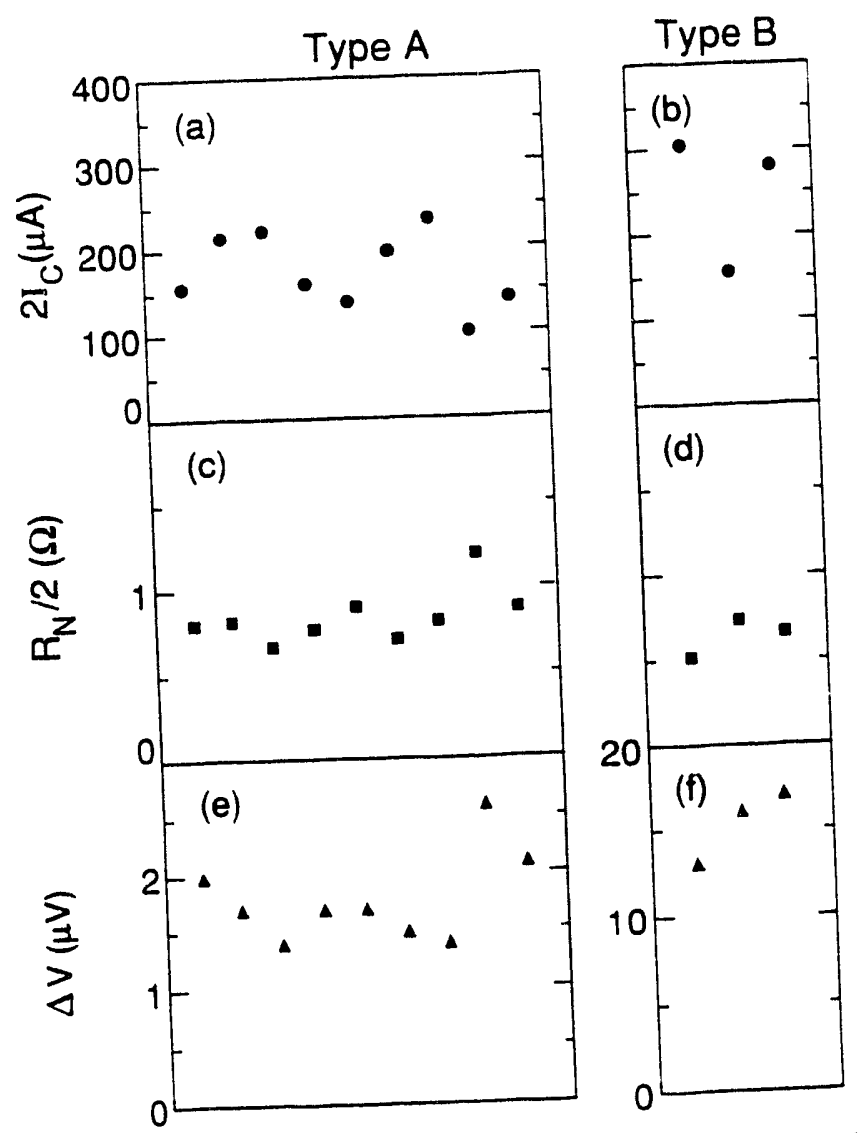

Fig. 1. Critical current (a) and (b); asymptotic resistance (c) and (d); and peak-to-peak voltage modulation, $\Delta V ;(e)$ and (f) at $77 \mathrm{~K}$ for $12 \mathrm{SQUID}$ on a single bicrystal. Abscissa enumerates the SQUIDs.

sured parameters are thus more than adequately reproducible, and all 12 SQUIDs operated in an entirely satisfactory way.

Figure 2 shows the spectral density of the flux noise measured in a Type B SQUTD in a flux-locked loop. The white noise is remarkably low, $\operatorname{ss}(1 / 2, \Phi)(f) \approx$ $6 \mu \Phi_{0} \mathrm{~Hz}^{-1 / 2}$, corresponding to a noise energy $\varepsilon=$ $\mathrm{S}_{\Phi}(\mathrm{f}) / 2 \mathrm{~L}=2 \times 10^{-30} \mathrm{JHz} \mathrm{H}^{-1}$. However, there is substantial $1 / \mathrm{f}$ noise at frequencies below about $1 \mathrm{kHz}$. As also shown in Figure 2, however, this noise power was reduced at low frequencies by two orders of magnitude by a scheme in which the current-bias was reversed at about $2 \mathrm{kHz}$. This reduction implies that the $1 / \mathrm{f}$ noise arose from fluctuations in the critical current of the junctions.

A magnetometer was constructed by coupling a single loop of YBCO to the body of a Type A SQUID. The loop, which was patterned in the same step as the SQUID, increased the effective magnetic field pick-up area of the magnetometer by a factor of about 22 . The

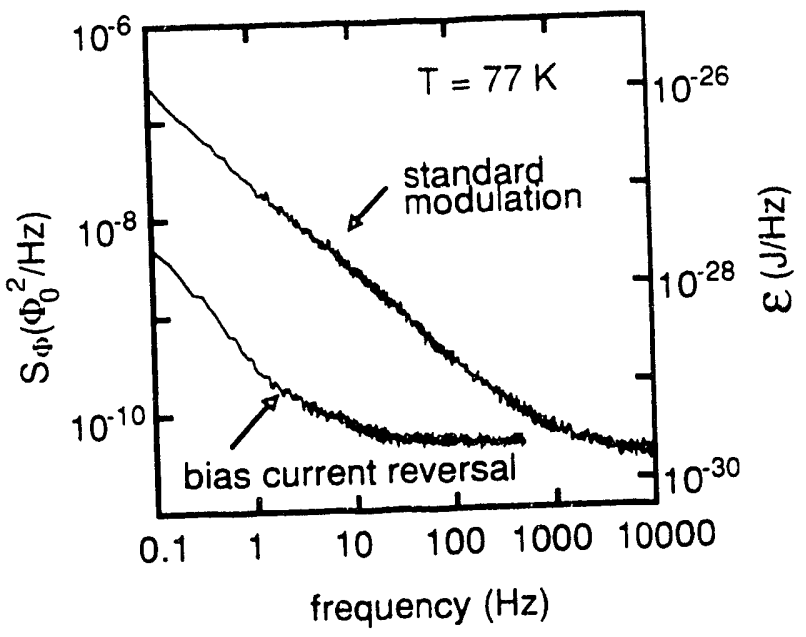

Fig. 2. Flux noise spectral density, $S_{\Phi}(f)$, and noise energy, $\varepsilon(f)$, for type B SQUID (500 $\mu \mathrm{m}$ outer dimension) at $77 \mathrm{~K}$. Upper trace is for conventional flux-locked loop with static current bias, lower trace is for bias reversal scheme.

measured magnetic field noise was about $300 \mathrm{fT} \mathrm{Hz}^{-1 / 2}$ at frequencies down to $0.5 \mathrm{~Hz}$. This is the highest sensitivity yet reported for any dc SQUID magnetometer operating at $77 \mathrm{~K}$. Preliminary experiments have been made on a magnetometer with a multiturn flux transformer. The white noise was about $30 \mathrm{fT} \mathrm{Hz}^{-1 / 2}$ an order of magnitude improvement over that of the single-turn magnetometer, but the l/f noise was still higher than desirable. Work is in progress to reduce the level of this noise.

In summary, we have developed a simple, highly reproducible magnetometer with adequate sensitivity for geophysical applications and nondestructive testing. It is expected that these magnetometers will be incorporated into dewars suitable for geophysical applications in FY93, and tests of their performance made in the field.

\section{Publications}

A.H. Miklich, D. Koelle, E. Dantsker, D.T. Nemeth, J.J. Kingston, R.F. Kromann, and J. Clarke, "Bicrystal YBCO dc SQUIDs with Low Noise," to be published in Proceedings of the 1992 Applied Superconductivity Conference, Chicago, IL, August 23-28, 1992.

A.H. Miklich, D. Koelle, E. Dantsker, D.T. Nemeth, F. Ludwig, J.J. Kingston, and J. Clarke, "Sensitive dc SQUID Magnetometers Made of Single Layers of $\mathrm{Yba}_{2} \mathrm{Cu}_{3} \mathrm{O}_{7-x}$," abstract for the March Meeting of the American Physical Society, March 22-26, 1993. 
Novel Surface and Interface Studies Using Photoelectron Spectroscopy Combined with Scanning Tunneling Microscopy

Principal Investigator: Charles S. Fadley

Funding: $\$ 129,000$ (FY92) $\$ 193,900$ (FY91)

\section{Project Description}

This project emphasizes developing new methods for the study of surfaces, interfaces, and nanostructures and applying these methods to novel surface morphologies that have been synthesized as part of the research. A principal focus is on experimental characterization techniques utilizing synchrotron radiation and combining synchrotron radiation measurements withi either in situ or independent characterizations by other complementary techniques such as scanning tunneling microscopy in unique ways.

The principal experimental methods used are photoelectron spectroscopy and photoelectron diffraction with high resolutions in both energy and emission angles, which together provide information on both the atomic geometries of surface species in different chemical or magnetic states and the valence electronic structure that binds them together. Another recent development that we are pursuing is the analysis of photoelectron diffraction data by holographic methods; this promises to produce true three-dimensional images of near-surface structure. State-of-the-art equipment is available for performing such angleresolved diffraction studies, and we are presently working on a next-generation system. This new system will provide higher resolutions and higher data acquisition rates, and will permit detecting electrons at extremely high rates, as well as measuring spinresolved electron spectra in studies of magnetic systems. Another instrumental development underway is the combination of photoelectron diffraction and scanning tunneling microscopy, two highly complementary surface structure probes, in one ultrahigh vacuum system. An additional important component of the work is also the theoretical interpretation of photoelectron diffraction patterns and the development of more efficient and accurate methods of extracting accurate structural information from them. This involves unique computer codes for calculating diffraction patterns, as well as for holographically inverting these patterns to produce realspace images.

\section{Accomplishments}

Experimental and Theoretical Studies of the Possibility of Surface Structure Determinations from Electron Emission Holography. The intriguing prospect of being able to use electron diffraction patterns above surfaces to determine surface atomic structures directly by holographic imaging methods is a very recent idea. Our group is still one of relatively few in the world working to assess the feasibility of this approach. Our experimental studies of both the bulk semiconductors $\mathrm{Si}$ and $\mathrm{Ge}$ and a simple adsorbate system (S/Ni) show that holographic images do indeed contain features associated with near-neighbor atoms. These images are distorted and shifted by various effects in the strong electron-atom scattering, but we and others have suggested several methods that have been demonstrated to improve image quality. Although bulk images are found to be more difficult to correct fully, for the adsorbate test case of $S / \mathrm{Ni}$, we have produced the most accurate nearest-neighbor images from experimental data to date. This adsorbate work has also suggested several procedures that should yield even more accurate images in the future using holograms at several energies excited by high-brightness synchrotron radiation. In addition, we have theoretically explored the possibility of using spinpolarized photoelectron holography for imaging magnetic moments, and this method also shows promise for future studies.

Development of an Ultrahigh Vacuum Scanning Tunneling Microscope and Application of It to Metal-Semiconductor Interactions. In preparation for combining scanning tunneling microscopy with photoelectron diffraction and holography, we have continued to use a custombuilt ultrahigh vacuum scanning tunneling microscope (STM). This system provides for in situ transfer of specimens from the STM to stations for either metal deposition, ion bombardment, or LEED/Auger characterization. As a first system of study with it, we have chosen the interaction of $\mathrm{Sn}$ with $\mathrm{Ge}(111)$ and obtained high-quality images with atomic resolution for several types of structures including: the clean $c(2 \times 8)$ surface, room-temperature-deposited $\mathrm{Sn}$ atoms and microclusters, and an annealed $\mathrm{Sn} / \mathrm{Ge}$ structure that is very similar to the well-known $(7 \times 7)$ reconstruction on Si(111). These images furthermore contain several new structural elements not observed before: co-existent $c(2 \times 8)$ structures that possess both symmetric adatoms and asymmetric adatoms, a $(5 \times 5)$ structure that forms in large domains co-existent with the $(7 \times 7)$ structure, and the indication that $\mathrm{Sn}$ preferentially occupies adatom positions in the $(5 \times 5)$ and $(7 \times 7)$ structure that are away from the large comer holes of the unit cell. 
Combination of Photoelectron Diffraction and Scanning Tunneling Microscopy in a Single System. We have completed the design and construction of a custonbuilt chamber and special sample manipulator that will be added to an existing photoelectron spectrometer so as to permit performing for the first time photoelectron diffraction and scanning tunneling microscopy measurements on the same sample. This system will be completed in FY93.

Other Surface Structure Studies Based Upon Photoelectron Diffraction. High-temperature surface phase transition on $\mathrm{Ge}(111)$ : This transition occurs $150^{\circ}$ below the melting point. Our results include holographic analyses below and above the transition temperature, and they indicate that the transition involves a layer about 2 atoms in thickness, a conclusion that is in good agreement with other techniques and which has further clarified the nature of what appears to be a surface "pre-melting" transition.

Diffraction of inelastic photoelectrons: This has also been studied for $\mathrm{Al}, \mathrm{Ge}$, and $\mathrm{W}$ and found to be much suppressed along low-index directions due to multiple scattering effects that we have successfully modeled theoretically. Such effects could be useful in locating low-index directions in epitaxial layers.

Diffraction of energy-integrated valence photoelectron spectra: This has been studied for $W$ and found to exhibit both direct-transition effects (Brillouin zone selectivity) at lower temperatures and with lower excitation energies, and atomic-orbital angular momentum effects for higher temperatures and higher energies.

More efficient methods for the theoretical simulation of photoelectron and Auger electron diffraction results: This work is centered on a general-purpose program that we have written for doing both singlescattering and fully-converged multiple-scattering calculations of such diffraction patterns. The approach is based on a new separable Green's function method. During the current year, the speed of this code has been increased by over an order of magnitude.

Spin-polarized photoelectron diffraction: The addition of spin resolution to the photoelectron diffraction measurement has also been studied theoretically in both a single-scattering and multiple scattering framework. As noted above, the holographic analysis of this type of data shows promise for directly imaging magnetic moments.

Oxygen on Si(111): Preliminary photoelectron diffraction data have been obtained for thin (approximately 5 $\AA$ ) thermal oxide layers on $\mathrm{Si}(111)$, with the oxide $\mathrm{Si}$ signal being resolved from that of the underlying elemental Si. These data suggest for the first time that the oxide has a degree of crystalline order, and this work will be pursued further in the coming year.

\section{Publications}

S. Thevuthasan, G.S. Herman, R.S. Saiki, A.P. Kaduwela, and C.S. Fadley, "Electron Emission Holography: Prospects and Limitations," Phys. Rev. Lett. 67, 469 (1991).

A.P. Kaduwela, D.J. Friedman, and C.S. Fadley, "Application of a New Multiple-Scattering Method to Photoelectron Diffraction and Auger Electron Diffraction," J. Electron, Spectrosc. 57, 223 (1991).

G.S. Herman, S. Thevuthasan, T.T. Tran, Y.J. Kim, and C.S. Fadley, "Imaging of Near-Neighbor Atoms in Semiconductors by Photoelectron Holography," Phys. Rev. Lett. 68, 650 (1992).

G.S. Herman, T.T. Tran, K. Higashiyama, and C.S. Fadley, "Valence Photoelectron Diffraction and Direct Transition Effects," Phys. Rev. Lett. 68, 1204 (1992).

S. Thevuthasan, G.S. Herman, A.P. Kaduwela, T.T. Tran, Y.J. Kim, RS. Saiki, M.A. Van Hove, and C.S. Fadley, "Electron Emission Holography of Small Clusters and Surfaces," J. Vac. Sci. and Tech. A10, 2261 (1992).

T.T. Tran, S. Thevuthasan, Y.J. Kim, G.S. Herman, D.J. Friedman, and C.S. Fadley, "Photoelectron Diffraction and Photoelectron Holography Study of a Ge(111) High-Temperature Surface Phase Transition," Phys. Rev. B45, 12106 (1992).

R.S. Saiki, A.P. Kaduwela, Y.J. Kim, and C.S. Fadley, "Test of High-Resolution X-Ray Photoelectron Diffraction and Holographic Imaging for $\mathrm{c}(2 \times 2) S$ on $\mathrm{Ni}(001), "$ Surf. Sci. , in press.

R.S. Saiki, A.P. Kaduwela, J. Osterwalder, D.J. Friedman, C.S. Fadley, and C.R. Brundle, "X-Ray Photoelectron Diffraction and Low Energy Electron Diffraction Study of the Interaction of Oxygen with the $\mathrm{Ni}(001)$ Surface: from $\mathrm{c}(2 \times 2)$ o to Saturated Oxide," Surf. Sci., in press.

K. Higashiyama, S.K. Lewis, R.X. Ynzunza, E.D. Tober, Y.J. Kim, and C.S. Fadley, "Asymmetry of Adatoms on Ge(111) Observed by Scanning Tunneling Microscopy: The Clean $\mathrm{c}(2 \times 8)$ and $\mathrm{Sn}$-induced $(7 \times 7)$ Structures," Surf. Sci., in press.

G.S. Herman, E.L. Bullock, M. Yamada, A.P. Kaduwela, D.J. Friedman, S. Thevuthasan, Y.J. Kim, T.T. Tran, C.S. Fadley, Th. Lindner, D.E. Ricken, A.W. 
Robinson, and A.M. Bradshaw, "A Scanned-Angle and Scanned-Energy Photoelectron Diffraction Study of $\mathrm{R}(3) \times \mathrm{R}(3) \mathrm{R} 30^{\circ} \mathrm{Ag}$ on $\mathrm{Si}(111)$," Surf. Sci., in press.

T.T. Tran, S. Thevuthasan, Y.J. Kim, D.J. Friedman, A.P. Kaduwela, G.S. Herman, and C.S. Fadley, "Photoelectron Diffraction and Holography Study of a $\mathrm{Ge}(111)$ High-Temperature Surface Phase Transition," Surf. Sci., in press.

B.D. Hermsmeier, B. Sinkovic, C.S. Fadley, M.O. Krause, J. Jiminez-Mier, P. Gerard, T.A. Carlson, and S.T. Manson, "Energy Dependence of the Outer CoreLevel Multiplet Structures in Atomic Mn and MnContaining Compounds," Phys. Rev. B (accepted for publication).

S. Thevuthasan, R.X. Ynzunza, E.D. Tober, and C.S. Fadley, "High-Energy Photoelectron Holography for an Adsorbate Test System: $c(2 \times 2) S$ on Ni(001)," submitted to Phys. Reo., Rapid Communications.

A.P. Kaduwela, Z. Wang, S. Thevuthasan, M.A. Van Hove, and C.S. Fadley, "Spin-Polarized Photoelectron Holography and the Possibility of Imaging ShortRange Magnetic Order," submitted to Phys. Rev. Lett.

C.S. Fadley, in Synchrotron Radiation Research: Advances in Surface and Interface Science, "Surface Structure Studies Using Photoelectron Diffraction and Auger Electron Diffraction," R.Z. Bachrach, Ed. (Plenum Press, New York, 1992), invited review.

C.S. Fadley, "Diffraction and Holography with Photoelectrons and Auger Electrons: Some New Directions," invited review article to appear in Surface Science Reports.

\section{Ceramic-Ceramic Joining via a Transient Liquid Phase}

Principal Investigators: Andreas M. Glaeser and Rowland M. Cannon

Funding: $\$ 60,000$

\section{Project Description}

Recent progress in the processing of advanced ceramics has resulted in a more widespread acceptance of ceramics as candidates for demanding structural and electronic applications. For many of these projected applications, reliable and cost-effective joining techniques must be available. Ceramic-ceramic and ceramic-metal joining research has focused primarily on solid-state methods (diffusion/ pressure bonding) or liquid-based methods (brazing). Neither method is without its difficulties and limitations. Diffusion bonding is expensive, only flat specimens can be joined, a vacuum/inert atmosphere is required, and pressure must be applied. The need to apply pressure during diffusion bonding imposes restrictions on the joint geometry. Although brazing is more attractive commercially, the brazes that have been developed for ceramic-ceramic and ceramic-metal joining melt at relatively low temperatures $\left(<1000^{\circ} \mathrm{C}\right)$, precluding high-temperature applications. The large thermal expansion mismatch with ceramics that accompanies the low melting point can induce failure in assemblies subjected to thermal cycling. A practical joining method that provides refractory, thermal expansion matched joints was lacking.

This research is intended to explore a novel approach to ceramic-ceramic and ceramic-metal joining, an approach utilizing microdesigned metal foils that develop a transient liquid phase (TLP). The method relies on the deposition of a thin film $(e .8 ., 1-5 \mu \mathrm{m})$ of a low melting point metal or alloy onto a much thicker foil (e.8., 25-250 $\mu \mathrm{m})$ of a more refractory metal or alloy as illustrated in Figure 3. The two metals or alloys are selected such that the more refractory metal incorporates the less refractory component and produces a suitably refractory alloy. Ideally, such a method melds the best features of solid-state and liquid-based joining methods. The low melting point thin film mimics brazing and relaxes constraints imposed on surface preparation. By design, only a small amount of liquid is formed, and it is formed only where it is needed. Diffusion of the less refractory metal into the refractory host, reaction of the less refractory with the refractory host, or a combination of these processes can lead to the disappearance of the liquid. If necessary, pressureless annealing at higher temperatures can be used to fully homogenize the

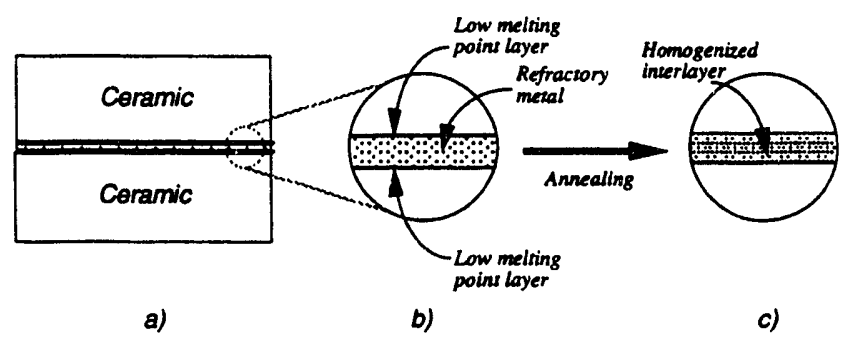

Fig. 3. Schematic illustration of (a) ceramic/ interlayer/ceramic assembly, (b) detail of microdesigned interlayer prior to heating, and (c) uniform interlayer after bonding and heat treatment. 
composition by interdiffusion. In conventional brazing, the melting point of the brazing alloy must exceed the ultimate use temperature; for high-temperature applications, this implies even higher temperature joining. Using a TLP, the joint is formed at low temperature, and processing is carried out at temperatures less than or equal to the ultimate use temperature. This minimizes degradation of the ceramic during bonding, and in cases where ceramics are bonded to temperature sensitive structural metals, avoids overaging of the metal constituent. The high melting point metal dominates the melting characteristics of the ultimately uniform interlayer, and can be selected to match the thermal expansion characteristics of the ceramic. The former allows the component to be used at high temperature; the latter minimizes thermal expansion mismatch and associated fatigue and failure if the assembly is thermally cycled.

Our objectives were to: (1) explore the use of $\mathrm{Cu} / \mathrm{Ni} /$ $\mathrm{Cu}$ and $\mathrm{Cu} / \mathrm{Pt} / \mathrm{Cu}$ multilayer interlayers as a basis for TLP brazing of $\mathrm{Al}_{2} \mathrm{O}_{3}$; (2) conduct a fundamental study of the chemical, microstructural, and mechanical characteristics of $\mathrm{Cu}-\mathrm{Ni}$ and $\mathrm{Cu}-\mathrm{Pt}$ TLP-bonded $\mathrm{Al}_{2} \mathrm{O}_{3}$; and (3) seek to develop TLP bonding routes for joining zirconia ceramics to themselves and to structural steels via metal interlayers. It was felt that if the method proved successful, the research would establish a new method of ceramic-ceramic and ceramic-metal joining. It was anticipated that the initial application and extension of the method would be in the structural ceramics area, with a view toward materials of interest in high-temperature energy conversion systems. However, it was felt that other areas could also be beneficially impacted.

\section{Accomplishments}

Several processing and performance advantages were expected from utilization of this approach. Key among these was a substantial reduction in processing temperature. This minimizes degradation of the ceramic during bonding, and in cases where ceramics are bonded to temperature sensitive structural metals, avoids overaging of the metal constituent. Ceramic degradation is a key problem for assemblies involving transformationally-toughened zirconia and silicon nitride. The high melting point metal dominates the melting characteristics of the ultimately uniform interlayer, and can be selected to match the thermal expansion characteristics of the ceramic. The former allows the component to be used at high temperature; the latter minimizes thermal expansion mismatch and associated fatigue and failure of thermally cycled assemblies.
During the period of this research program, $\mathrm{Al}_{2} \mathrm{O}_{3} /$ $\mathrm{Al}_{2} \mathrm{O}_{3}$ tonds were produced using three different metal interlayer chemistries - the originally proposed $\mathrm{Cu} / \mathrm{Pt}$, $\mathrm{Cu} / \mathrm{Ni}$, and in addition, $\mathrm{Cu} / \mathrm{Nb}$. In all cases, the bonding temperature $\left(1150^{\circ} \mathrm{C}\right)$ was several hundred degrees lower than those required to produce strong $\mathrm{Al}_{2} \mathrm{O}_{3} / \mathrm{Al}_{2} \mathrm{O}_{3}$ bonds with $\mathrm{Pt}, \mathrm{Ni}$, and $\mathrm{Nb}$ interlayers via conventional joining methods. Room temperature mechanical tests of these joined assemblies have demonstrated that strong ceramic/metal interfaces can be formed using PTLP bonding. In several instances, failure occurred within the ceramic component, indicating that the ceramic, not the ceramic/metal interface was the weak mechanical link in the structure. Microstructural studies of the fracture surfaces indicate that good ceramic/metal interfacial contact was achieved during bonding. The results suggest that if the processing can be improved further, ceramic failure rather than ceramic/metal interfacial failure may become the dominant fracture mode.

Microchemical characterization of $\mathrm{Cu} / \mathrm{Pt} / \mathrm{Cu}$ interlayers showed that substantial interdiffusion occurred, producing a Pt-rich interlayer. If necessary, pressureless annealing at higher temperatures could be used to more fully homogenize the interlayer. High room temperature flexure strengths were obtained. Prior studies have shown that diffusion-bonded $\mathrm{Al}_{2} \mathrm{O}_{3} / \mathrm{Pt} / \mathrm{Al}_{2} \mathrm{O}_{3}$ ensembles retain a significant portion of their room temperature strength to temperatures of $1200^{\circ} \mathrm{C}$, and similar results are anticipated for the PTLP joined assemblies. Results for $\mathrm{Cu} / \mathrm{Ni}$ bondod assemblies are expected to be qualitatively similar, with lower potential use temperatures. Microchemical characterization of $\mathrm{Cu} / \mathrm{Nb}$ bonded assemblies indicate that other mechanisms for consuming the liquid former can act, again yielding strong refractory joints.

The aforementioned results were hoped for, and to some extent anticipated. An interesting and unanticipated result was the indication that profound changes in strength could be achieved by post-bonding hightemperature pressureless annealing. In the $\mathrm{Cu} / \mathrm{Pt}$ system, $10 \mathrm{hr}$ post-bonding anneals in air at $1000^{\circ} \mathrm{C}$ appear to have a beneficial effect on the mechanical behavior, possibly through an effect of a change in the near-interfacial chemistry on adhesion and thereby the ceramic-metal interfacial strength. Annealing of $\mathrm{Cu} / \mathrm{Pt}$ bonded assemblies in oxygen-gettered argon appeared to cause interfacial dewetting and loss of strength. In contrast, in $\mathrm{Cu} / \mathrm{Nb}$ bonded assemblies, an identical heat treatment in oxygen-gettered argon had no deleterious effect. Thus, studies of environmental effects on ceramic/ metal interfacial microstructure and properties emerged as an important new research direction. 
Efforts to develop methods of joining zirconia were just being initiated at the time this period of research support ended. This research focused on $\mathrm{Cu} / \mathrm{Pd}, \mathrm{Au} /$ $\mathrm{Pd}$, and $\mathrm{Cu}-\mathrm{Au} / \mathrm{Pd}$ interlayers.

\section{Publications}

M.L. Shalz, B.J. Dalgleish, A.P. Tomsia, and A.M. Glaeser, "Ceramic Joining; I. Partial Transient Liquid Phase Bonding of Alumina via $\mathrm{Cu} / \mathrm{Pt}$ Interlayers," J. Mat Sci., in press.

M.L. Shalz, B.J. Dalgleish, A.P. Tomsia, and A.M. Glaeser, "Ceramic Joining; II. Bonding of Alumina via Microdesigned $\mathrm{Cu}-\mathrm{Nb}$ Interlayers," in preparation.

M.L. Shalz, B.J. Dalgleish, and A.M. Glaeser, "Ceramic Joining; III. Partial Transient Liquid Phase Bonding of Alumina via $\mathrm{Cu} / \mathrm{Ni}$ and $\mathrm{Cu} / 80 \mathrm{Ni2} 2 \mathrm{Cr}$ Interlayers," in preparation.

M.L. Shaiz, B.J. Dalgleish, A.P. Tomsia, and A.M. Glaeser, "A New Route to Joining Ceramics for High Temperature Applications," to be published in Proceedings of the Second International Forum on Structural Ceramics Joining, November 1-4, 1992, San Francisco, CA, in preparation.

M.L. Shalz, B.J. Dalgleish, A.P. Tomsia, and A.M. Glaeser, "A Transient FGM Approach to CeramicCeramic Joining," to be published in Proceedings of the Second International Symposium on Functionally Gradient Materials, November 1-4, 1992, San Francisco, CA, in preparation.

\section{Transport Spectroscopy of Quantum} Dots

Principal Investigator: Paul L. McEuen

Funding: $\$ 114,800$

\section{Project Description}

With modern materials growth and microfabrication technologies, it is possible to fabricate submicron electron systems whose transport properties are regulated by basic quantization phenomenon. For example, studies of lithographically defined GaAs structures ("dots") containing fewer than 100 electrons have demonstrated the importance of charge quantization to transport and have yielded detailed pictures of a dot's energy level spectrum.

Such devices are in excellent model system for exploring the physics of nanometer-scale electron gases. In this project we researched the properties of these systems. In particular, we studied gated GaAs singledot and multiple-dot devices by transport spectroscopy. The goals are to understand in detail how the effects of confinement, electron-lectron interactions, and high magnetic fields influence the properties of an ultrasmall electron gas.

\section{Accomplishments}

The major goals were met. A dilution refrigerator system has been installed and tested and is now operational. In addition, a computer-controlled measurement system has been designed and installed. We have also successfully fabricated nanostructures. For example, one-dimensional channels have been iabricated for a time-of-flight experiment. These onedimensional channels are formed by a gated constriction in a two-dimensional electron gas. These constrictions, which are lithographically $\sim 0.2 \mu \mathrm{m}$ long and 0.4 $\mu \mathrm{m}$ wide, were patterned using electron-beam lithography.

Figure 4 shows the conductance of the constriction as a function of an external gate voltage $\mathrm{Vg}$. This gate voltage controls the effective width of the conducting channel. The stepwise increase observed in the conductance is direct evidence for one-dimensional transport. A step of height $2 e^{2} / h$ occurs whenever an

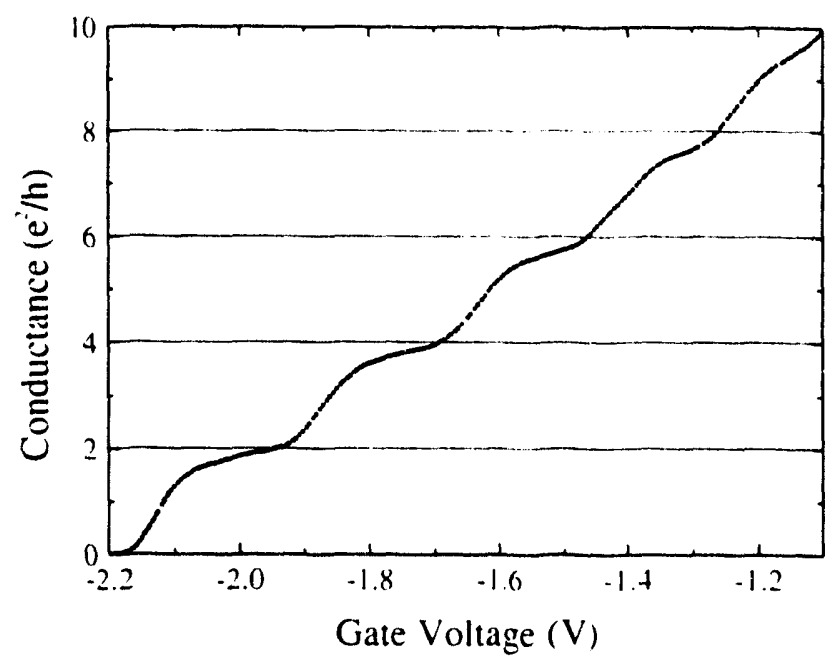

Fig. 4. Conductance vs. gate $V g$ of a narrow constriction in a two-dimensional electron gas at $T=0.3 \mathrm{~K}$. The steplike structure is direct evidence for the formation of onedimensional channels in the constriction. 
additional one-dimensional channel is occupied in the constriction, as is observed in the data. These devices are now being bonded to striplines for the time-offlight experiment. Preliminary measurements have been made and scientific results should be rapidly forthcoming.

\section{Publications}

C. Karadi, K. Wald, S. Jauhar, P.L. McEuen, and J. Orenstein, "Technique for Measurement of the Transit Time in a One-dimensional Edge State," abstract submitted to the March 1993 Meeting of the American Physical Society.

\section{Preliminary Studies of Quantitative Electron Detectors}

Principal Investigators: Michael A. O'Keefe, Kannen M. Krishnan, and Roar Kilaas

Funding: $\$ 40,000$

\section{Project Description}

Current options for achieving $1-\AA$ image resolution in a Quantitative Sub-Ångstrom Microscope (QSAM) involve image reconstruction (on- and off-line) based on focal image series using a medium-voltage TEM. Experimental tests of an advanced image reconstruction technique, extended-resolution electron microscopy (EREM), which has been developed at LBL, will be conducted on a suitable electron microscope. For such reconstruction techniques to work, it is essential that suitable quantitative detectors be developed.

Two unique instrumentation projects have been identified that will revolutionize the field of electron microscopy. One of these instruments, the QSAM, would seek to break through the $1-\AA$ image resolution barrier, while the other, the Magnetic Materials Microscope (MMM), would be able to resolve magnetic, chemical, and physical microstructures simultaneously at the nanometer scale of resolution. Both instruments will emphasize the quantitative recording and interpretation of images, diffraction patterns, and in-plane magnetization. The success of these projects will depend on the development of suitable rapidresponse quantitative electron detectors sensitive to the relevant elastic, inelastic, and magnetic scattered electron intensities.
The purpose of this project is to initiate development and testing of two detector designs for measurement of (1) high-resolution-image intensities (using a CCD camera with 16-bit dynamic range) and (2) in-plane magnetization (using a differential phase contrast (DPC) detector with picoamp sensitivity).

\section{Accomplishments}

In addition to the $C C D$ detector for high-resolutionimage recording, and the pure DPC detector for measurement of in-plane magnetization, we considered the possibility of a hybrid detector that would use a small fast-readout CCD to detect in-plane magnetization by measuring any position changes in the center of mass of the electron beam due to magnetic deviations as it was scanned across the magnetic sample; a hybrid detector using a $128 \times 128$-pixel CCD would have improved precision over a 9-sector DPC detector. Initial figures indicated that the rate of computations required was feasible, especially if some integrations were to be performed in hardware by reading out the CCD chip with appropriate binning.

Existing high-resolution electron microscope image detectors use either a phosphor powder or a scintillator such as YAG to convert electron energy to light for detection in a TV camera or CCD camera. A sample of a novel type of scintillator was obtained from a consultant and tested in one of the NCEM electron microscopes. This scintillator consisted of a fiber-optic plate in which the core glass of each fiber formed the scintillating material. It was hoped that this scintillator would increase the brightness obtained without degrading detector resolution. Tests at 800 $\mathrm{keV}$ and $1500 \mathrm{keV}$ showed that the scintillator was much brighter than the usual YAG, but that the increase in brightness did carry a penalty in resolution. Future tests may produce a way around this problem by using a scintillating fiber-optic with only the top few microns active.

\section{Time Domain Spectroscopy of Semi- conductors and Superconductors}

Principal Investigator: Joseph Orenstein

Funding: $\$ 176,000$

\section{Project Description}

Many of the most interesting and important phenomena in condensed matter physics are associated 
with energy scales in the terahertz (1012) regime of the electromagnetic spectrum. Coherent terahertz spectroscopy, the generation and detection of sub-picosecond electrical pulses, promises to open this exciting regime for study. A unique facility for coherent terahertz spectroscopy has been developed LBL, and the first results can be seen from the new spectrometers that we have designed and built.

Coherent terahertz spectroscopy requires the generation and detection of sub-picosecond electrical pulses. The source is based on the $\mathrm{F}$ t.jtoexcitation of a fast-response photoconductor such as radiation-damaged silicon. Excitation of the voltage-biased photoconductor by an ultrashort optical pulse from a laser induces a current spike. This spike in tum generates a sub-picosecond electromagnetic pulse that is radiated into free space. The coupling of the pulse to free space is increased with a lithographically fabricated antenna. The same device functions as a detector if the antenna terminals are connected to a current meter rather than a voltage source. The temporal profile of an electrical pulse transmitted through space is measured by varying the time-delay between the laser pulses that reach the generator and detector.

In addition to generation and detection a successful spectroscopic technique requires directed propagation of radiation in order to measure transmission coefficients of materials of interest. Figure 5 illustrates the terahertz spectrometer that we have designed and constructed. The key optical components are off-axis parabolic mirrors that collect the terahertz radiation, focus it onto the sample, and then redirect the energy toward the detector. The important characteristics of the spectrometer are listed below:
Spot size in the focal plane: Diffraction limited with f/1 optics

Signal/Noise ratio: Greater than 104

Spectral range: $\quad 0.10-1.8 \mathrm{THz}$

\section{Accomplishments}

In the past year we have demonstrated the power of terahertz spectroscopy in all of the projects that we have begun. In the following we describe the results of three projects: (1) quasiparticle dynamics in high- $T_{c}$ superconductors; (2) broadband spectroscopy of Josephson junction SIS detectors; and (3) dynamics of electron propagation in nanostructures.

Quasiparticle Dynamics in High- $T_{c}$ Superconductors. In a superconductor at absolute zero $(\mathrm{T}=0)$ all of the electrons are paired and Bose condense into a charged superfluid. Above $\mathrm{T}=0$, thermal agitation breaks apart some of these pairs - the unpaired electrons are known as "quasiparticles." Their coexistence with the condensate is the microscopic basis of the phenomenological "two-fluid model" for the dynamical properties of superconductors. The properties of quasiparticles, for example their scattering rate, are important for a theoretical understanding of high- $T_{c}$ superconductivity. Ordinary low frequency measurements, such as resistivity and Hall effect, are inapplicable because the superconducting electrons constitute a parallel transport channel which "shorts out" the quasiparticle resistance. Measurements at high frequency are capable of probing the quasiparticle response.

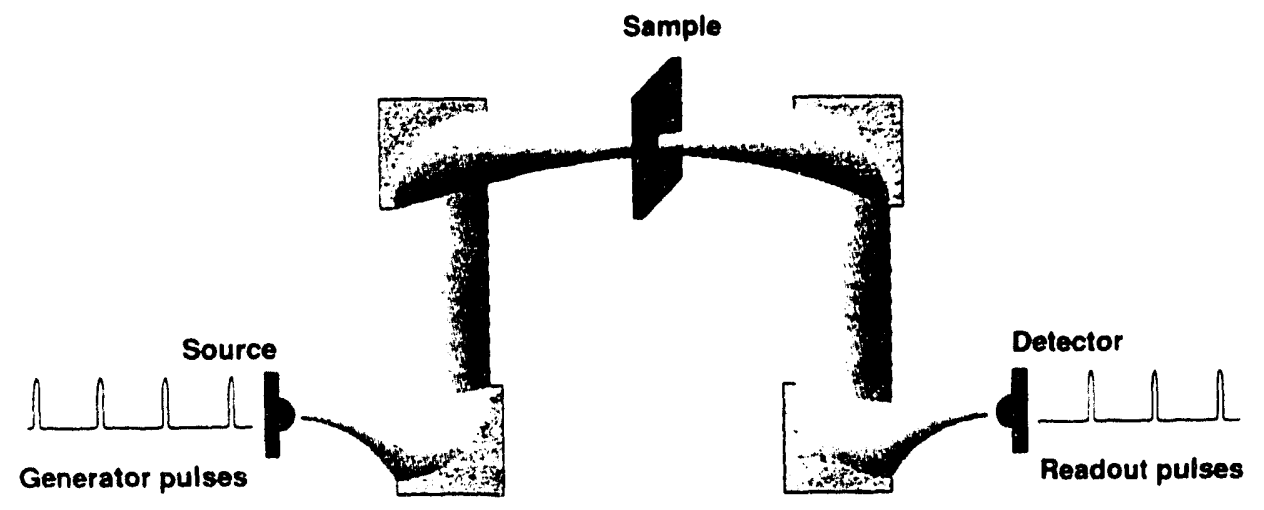

Fig. 5. Illustration of the terahertz spectrometer built in our laboratory. The semicircles on the source and detector chips represent silicon lenses which initially collimate the terahertz pulses into beams. Because the electromagnetic wavelengths are in the millimeter range, the beams spread rapidly due to diffraction. The paraboloidal mirrors, shown in gray, serve to recollimate the energy and send it on its way through the sample under test to the detector. 
Figure 6 shows the temporal profile of our electric field pulse transmitted through the superconductor, both above and below $T_{c}$. Notice the dramatic difference between the shape and magnitude of the pulses as the critical temperature is traversed. From the amplitude change and phase shift we can determine both real and imaginary components of the complex frequencydependent conductivity $e()$. The imaginary part reflects the response of the superconducting electrons as they attempt to screen the incident fields from the bulk of the materials. The real part of $e()$ corresponds to the dissipation of energy due to the presence of unpaired electrons.

Broadband Spectroscopy of Josephson Junction SIS Detectors. Gigahertz and terahertz detectors based on superconductor-insulator-superconductor (SIS) Josephson junctions play a critical role in astrophysical investigations. In spite of a great deal of study their broadband response has not been adequately characterized because of the lack of a suitable terahertz source. The spectroscopic techniques that we have developed are ideally suited to measure the response of SIS detectors throughout their design region of operation.

During this past year we have constructed a new terahertz spectrometer designed for characterizing the response of SIS detectors. The new system is necessary because it is not possible to measure transmission

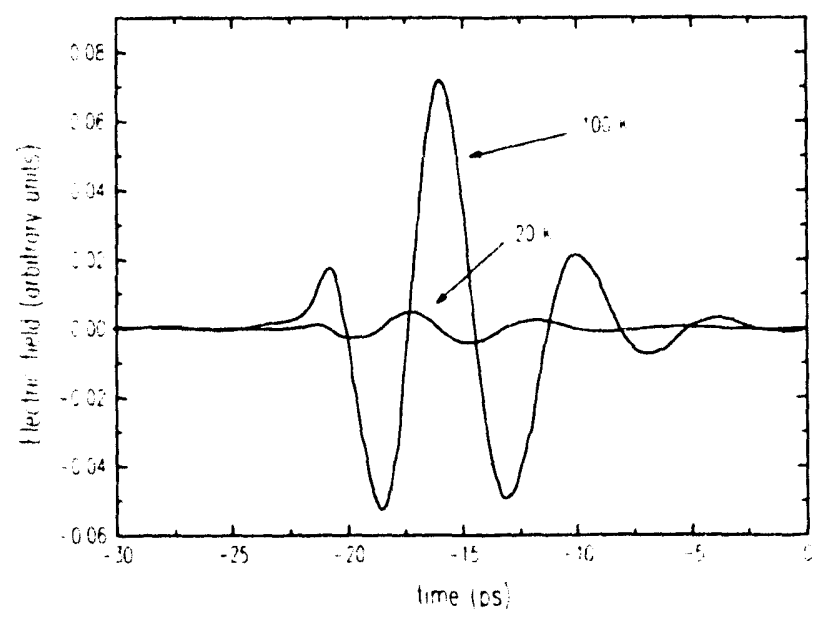

Fig. 6. The electric field pulse transmitted through a thin film of superconducting $\mathrm{YBa} 2 \mathrm{Cu} 3 \mathrm{O} 7$ both above and below the critical temperature. Notice the dramatic change in amplitude and phase of the two pulses. The pulse undergoes a dramatic reduction in magnitude and a nearly pi/2 phase shift in the superconducting state. through an SIS detector. Instead we have built a terahertz interferometer capable of directing two pulses onto the SIS device. Using this instrument we measure the cross-correlation function, that is, the current induced in the detector by two pulses, as a function of the time-delay between them. The Fourier transform of the cross-correlation yields the broadband response of the junction.

To date we have carried out an extensive measurement of the device in the linear regime. We have obtained the first broadband spectrum of an SIS device in the terahertz regime. In our future research we will focus on understanding the origin of the width of the spectral response near its threshold. In addition, we will study a new series of devices in which the junction area is reduced to $0.5(\mathrm{fm}) 2$ in order to reduce capacitance and enhance high-frequency response. Our spectroscopic technique is the only method capable of characterizing the response of these devices in this high-frequency regime.

Dynamics of Electron Propagation in Nanostructures. The two-dimensional electron gas formed by modulation doping of GaAs-GaAlAs heterojunctions forms an ideal system for studying transport in the quantum regime. Recently it has been dernonstrated that in the presence of a magnetic field these electrons are confined to one-dimensional states called "edge channels." Using terahertz pulses generated in our lab we are investigating, in the time domain, the dynamics of electron propagation in edge-channels.

Our experiments involve the propagation through edge channels interrupted by two constrictions or "doorways." Through application of a teraheriz pulse, a doorway can be momentarily opened to allow the passage of a burst of electrons. Their time of flight is measured by resolving their arrival of another constriction further downstream. At present we have fabricated doorway devices and are testing several methods for applying the electrical pulses.

Collaborations. All of our projects are undertaken in collaboration with other groups, at LBL, UC Berkeley, and outside institutions. The high- $T_{C}$ studies benefit from collaboration with the Hewlett-Packard Superconductivity Research Group, AT\&T Bell Laboratories, the Superconductivity Program of the Canadian Institute for Advanced Research. The SIS work is performed in collaboration with the group of Prof. Paul Richards at LBL. The research on the twodimensional electron gas in GaAs is performed in collaboration with Prof. Paul McEuen's group at LBL. 


\section{Femtosecond Dynamics in Condensed Matter}

Principal Investigator: Charles V. Shank

Funding: $\$ 123,700$ (FY92)

$\$ 268,200$ (FY91)

$\$ 296,500$ (FY90)

\section{Project Description}

The purpose of this project is to investigate ultrafast processes in condensed matter, including chemical reactions, coherenit vibrations, and electronic dephasing on a femtosecond time scale. The project includes development of a short-pulse laser source with the capability of generating 10 femtosecond pulses in the blue-green region of the spectrum. This unique laser system will extend femtosecond pulse generation, amplification, and compression techniques to wavelengths substantially shorter than those achieved to date. The system will provide the capability for investigating a unique class of molecules as well as other condensed matter systems that absorb in the blue-green region of the spectrum.

The laser system is based on a colliding-pulsemodelocked dye laser with two stages of amplification pumped by a XeF excimer laser. This system will produce optical pulses with durations of less that 50 femtoseconds over the spectral region from $400 \mathrm{~nm}$ to $600 \mathrm{~nm}$. Using pulse compression techniques, with an optical fiber to broaden the spectrum and chirp tine pulse, and a sequence of gratings and prisms to compensate the phase, it will be possible to achieve pulse durations on the order of 10 femtoseconds.

One particularly interesting class of molecules that we will be able to study with this unique laser system are the retinal proteins. One such protein, rhodopsin, is the light-sensitive molecule in the retina of the eye, and is the fundamental component of the visual system. This molecule efficiently converts photon energy into mechanical motion via a cis to trans conformational change or chemical reaction. The dynamics of this process are responsible for the incredible efficiency of the visual system and are thought to occur on a sub-picosecond time scale. Additional studies will be made on a related molecule, bacteriorhodopsin, which is responsible for the conversion of light energy to electrochemical energy by the generation of a transmembrane proton gradient. Studies of the speed of the cis-trans isomerization and the related vibrational dynamics of these proteins will provide inportant information about the efficiency of the chemical reaction and associated energy conversion.

\section{Accomplishments}

We have developed a new laser system to generate ultrashort pulses in the blue-green spectral region. Pulses of less that 20 femtoseconds have been generated over the spectral range from $450 \mathrm{~nm}$ to $550 \mathrm{~nm}$, with pulse energies on the order of $0.5 \mu \mathrm{J}$. These amplified pulses have been compressed to durations as short as 8 femtoseconds using an optical fiber with pairs of gratings and prisms for phase compensation. The extension of femtosecond pulse generation and compression techniques to shorter wavelengths provides the temporal resolution and photon energy necessary for investigating ultrafast processes in a variety of organic and solid state material systems.

The spectral dynamics of the primary event in vision have been resolved for the first time using the bluegreen laser system. The 11-cis retinal prosthetic group of rhodopsin is excited with a 35-femtosecond pump pulse at $500 \mathrm{~nm}$, and the transient changes in absorption are measured between $450 \mathrm{~nm}$ and $580 \mathrm{~nm}$ with a 10-femtosecond probe pulse. Within 200

femtoseconds, an increased absorption is observed between $540 \mathrm{~nm}$ and $580 \mathrm{~nm}$, indicating the formation of photoproduct on this time scale. These measurements demonstrate that the first step in vision, the 11cis $\rightarrow 11$-trans torsional isomerization of the rhodopsin chromophore, is essentially complete in only 200 femtoseconds. Our results further indicate an essentially barrierless transition in the formation of photoproduct, suggesting that the isomerization follows a nonadiabatic potential surface.

We have applied similar pump-probe techniques to investigate the vibrational dynamics associated with the trans-cis isomerization in bacteriorhodopsin. Timeresolved differential transmittance measurements reveal coherent vibrational oscillations resulting from impulsive excitation of the retinal chromophore as well as time-dependent changes reflecting its excited state dynamics and subsequent photochemistry... The nunstationary vibrational states persist on a picosecond time scale, sufficiently long for the frequencies to be resolved by Fourier analysis. Because of the rapid excited state reactive motion, the ground state reactant dynamics dominate the oscillatory response, a result that may serve as a guide for the interpretation of fast time-resolved measurements on other photochemically active systems. 


\section{Publications}

R.W. Schoenlein, J.-Y. Bigot, M.T. Portella, and C.V. Shank, "Generation of Blue-Green 10 fs Pulses Using an Excimer Pumped Dye Amplifier," Appl. Phys. Lett. 58 (1991).

R.W. Schoenlein, L.A. Peteanu, R.A. Mathies, and C.V. Shank, "The First Step in Vision: Femtosecond Isomerization of Rhodopsin," Science 2E:4, 412 (1991).

S.L. Dexheimer, Q. Wang, L.A. Peteanu, W.T. Pollard, R.A. Mathies, and C.V. Shank, "Femtosecond Impulsive Excitation of Nonstationary Vibrational States in Bacteriorhodopsin," Chem. Phys. Lett. 188, 61 (1992).

R.W. Schoenlein, Q. Wang, L.A. Peteanu, R.A. Mathies, and C.V. Shank, "Femtosecond Dynamics of the cistrans Isomerizati on of Isorhodopsin," in pi-paration.

L.A. Peteanu, R.W. Schoenlein, Q. Wang, R.A. Mathies, and C.V. Shank, "Broadband Spectral Dynamics of the cis-trans Isomerization of Rhodopsin," in preparation.

\section{Picometer-Scale Structure of Interfaces from Synchrotron Radiation}

Principal Investigators: Michel A. Van Hove, Charles S. Fadley, and Philip N. Ross

Funding: $\$ 137,400$

\section{Project Description}

Many physical, electronic, optical, magnetic, chemical, tribological, mechanical and other properties of modern materials repend intimately on their atomicscale structure. Modern materials increasingly rely on selected interfaces between chemically or structurally different substances. It is the aim of this project to develop novel methods that can determine the structure of solid/vacuum, solid/solid and solid/liquid interfaces on the subÅngström scale, by use of synchrotron radiation from LBL's Advanced Light Source.

We use the techriques of photoelectron diffraction, including forward focusing of electrons and electron holography, both of which are novel forms of photoelectron diffraction with great future potential.
These techniques are currently in a state of fundamental development and require extensive theoretical work as a proof of principle for complex structures, and to deliver subÅngström accuracy. Forward focusing and electron holography, in particular, show promises of great advances, especially in conjunction with synchrotron radiation. Our emphasis is on the development of these techniques for studying solid/ vacuum and solid/solid interfaces, as well as to extend the related technique of near-edge $x$-ray absorption spectroscopy to solid/liquid interfaces.

\section{Accomplishments}

In this project's first year, we have achieved significant advances toward the stated goals using both photoelectron diffraction and electron holography. Our state-of-the-art Rehr-Albers computer codes were accelerated by an order of magnitude, while also being made portable to workstations. Various holographic transformations that produce 3-dimensional atomic images from measured data and theoretical simulations were successfully implemented and tested with the simple but very useful case of a CO molecule bonded to an Fe surface (Figure "): this test case has illuminated the critical issues for extending the method to other interfaces.

Prominent among these is the role of forward focusing, which produces a strong electron emission peak downstream from an atom as seen from a photoemitting atom. The forward focusing peak provides useful directional information (thereby readily indicating interatomic directions), but it also disturbs the holographic information. The holographic interference between the directly emitter electron wave and the waves scattered by neighboring atoms allows one, by suitable Fourier transformation, to reconstruct a direct image of atomic positions. The forward focusing effect displaces and smears the atomic images. By removing the forward peak and correcting for phase shifts in other emission directions, we can achieve nearly undisplaced and unsmeared atomic images.

New experiments in photoelectron diffraction and holography have already been carried out and successfully interpreted for sulfur bonded to Ni surfaces. The S/Ni results have provided the most accurate holographic images obtained to date, with excellent agreement between experiment and theory. Similar analyses of spin-polarized data on surfaces of $\mathrm{Mn}^{2+}$ 


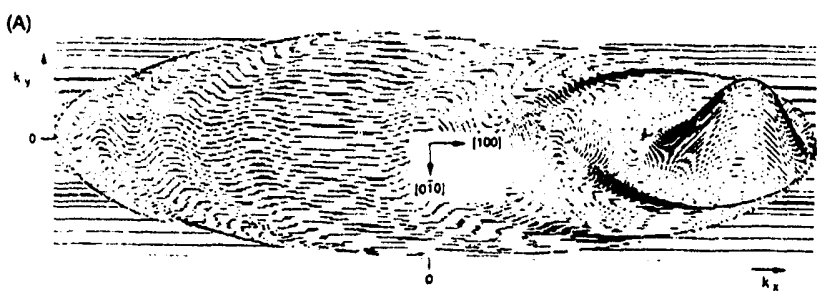

(B)

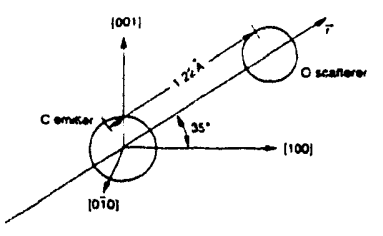

(C)

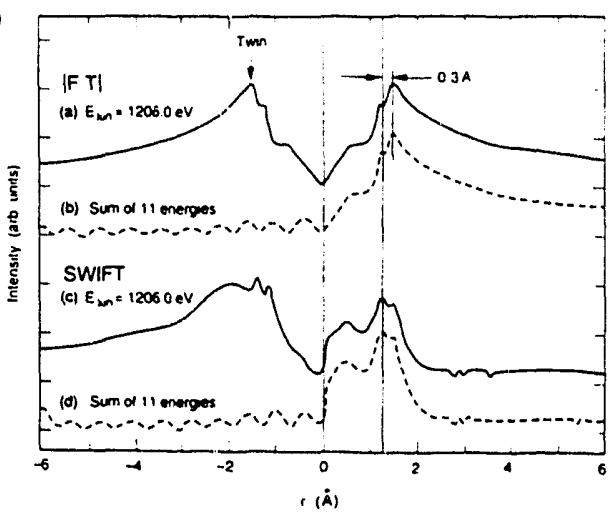

Fig. 7. Electron holographic reconstruction of a $\mathrm{CO}$ molecule imaged by photoemission from its carbon atom. Top panel $(A)$ shows a hologram consisting of a hemisphere of calculated emission intensity above a hypothetical horizontal surface to which the $\mathrm{CO}$ is bonded at an angle of $35^{\circ}$, as shown schematically in middle panel (B). Electrons with $1206 \mathrm{eV}$ kinetic energy emitted from $C$ are forward focused by the $O$ atom into the peak at top right, while interference betwein direct emission and scattering from $O$ produces interference fringes visible around that peak. Bottom panel (C) shows reconstruction by Fourier transformation of the oxygen atom, in the form of a "density plot" along the $\mathrm{CO}$ axis: the simple Fourier transform (a) produces a displaced, smeared peak $0.3 \AA$ away from the correct $O$ position (indicated by a vertical line), and a symmetrical twin image on the other side of the $C$ atom. Phased summation over similar holograms at different energies (b) removes the undesirable twin. A "scatteredwave-included Fourier transform" (SWIFT) corrects the $O$ position (c). Combining both corrections gives a very useful twin-free image of the oxygen atom (d). lattices have also been initiated with the goal of imaging short-range magnetic order. These results are very encouraging and suggest that the three-dimensional images of magnetic moments should be derivable from data that will be obtained with next-generation synchrotron radiation sources. Our successes on these fronts in the first funding year have taken precedence over progress in the area of the solid/liquid interface, which topic will be addressed in the second funding year.

\section{Publications}

S. Thevuthasan, G.S. Herman, A.P. Kaduwela, T.T. Tran, Y.J. Kim, R.S. Saiki, M.A. Van Hove and C.S. Fadley, "Electron Emission Holography of Small Clusters and Surfaces," J. Vac. Sci. Technol. A10, 2261 (1992).

S. Thevuthasan, R.X. Ynzunza, E.D. Tober, C.S. Fadley, A.P. Kaduwela and M.A. Van Hove, "High-Energy Photoelectron Holography for an Adsorbate Test System: c(2×2)S on Ni(100)," submitted to Phys. Rev. Lett.

A.P. Kaduwela, Z. Wang, S. Thevuthasan, M.A. Van Hove and C.S. Fadley, "Spin-Polarized Photoelectron Holography and the Possibility of Imaging ShortRange Magnetic Order," submitted to Phys. Reo.

A.P. Kaduwela, M.A. Van Hove and C.S. Fadley, "Comment on 'Adsorbate Structures from Photoelectron Diffraction: Holographic Reconstruction or RealSpace Triangulations?'," submitted to Phys. Rev. Lett.

C.S. Fadley, "Diffraction and Holography with Photoelectrons and Auger Electrons: Some New Directions," to appear in Surf. Sci. Rep.

C.S. Fadley, "Surface Structure Studies Using Photoelectron Diffraction and Auger Electron Diffraction," in Synchrotron Radiation Research: Adoances in Surface Science, R.Z. Bachrach, Ed., Plenum, New York (1992).

M.A. Van Hove, "Electron Holography," in preparation.

M.A. Van Hove, "LEED, Forward Focusing and Electron Holography," in MSA Bulletin 23, in press. 


\section{Processing of Semiconductor Materials under High Hydrostatic Pressure}

Principal Investigators: Wladyslaw Walukiewicz and Raymond Jeanloz

Funding: $\$ 50,000$

\section{Project Description}

We have developed a new approach to address the problem of low activation efficiency of shallow impurities in wide gap semiconductors. Our approach is based on recent experimental findings indicating that the formation energies of the defects responsible for the compensation of shallow donors or acceptors are strongly dependent on hydrostatic pressure. We therefore suggest that the application of external pressure can be used to intentionally control the concentration of native defects and thus also the concentration of electrically active uncompensated dopants.

The effectiveness of this process for a specific semiconductor material depends on the conduction and valence band pressure coefficients measured with respect to the common energy reference. In direct gap group III-V semiconductors such as GaAs or InP the pressure coefficient for the conduction band $\Gamma$ minimum is equal to about $0.1 \mathrm{eV} / \mathrm{GPa}$. This means that under the pressure of $4 \mathrm{GPa}$ the formation energy of triply ionized column III vacancies will be reduced by more than $1 \mathrm{eV}$. Consequently, annealing of n-type $\mathrm{GaAs}$ or InP under this high pressure will enhance the concentration of the acceptor-like vacancies, leading to a significant reduction of the donor activation efficiency in these materials.

The opposite behavior should be observed in p-type IVI compounds. In these materials valence band pressure coefficients are in the range of $-0.02 \mathrm{eV} / \mathrm{GPa}$ to $-0.1 \mathrm{eV} / \mathrm{GPa}$. The negative values of the pressure coefficients indicate that under hydrostatic pressure the valence band edges are shifted toward the native defect levels. This results in an increase of the formation energy of native donors compensating intentionally introduced acceptors. In ZnSe with the pressure coefficient of $-0.06 \mathrm{eV} / \mathrm{GPa}$ one needs only a pressure of $4 \mathrm{GPa}$ to dramatically reduce the equilibrium defect concentration. It is predicted that in $\mathrm{ZnSe}$ concentrations of free holes as high as $10^{19} \mathrm{~cm}^{-3}$ should be possible.

We have proposed to use diamond anvil cell technology to apply high hydrostatic pressure to the semicon- ducting materials. Pressures up to $8 \mathrm{GPa}$ and temperatures as high as $1100 \mathrm{~K}$ are required for the proposed annealing of n-type GaAs and p-type $\mathrm{ZnSe}$.

\section{Accomplishments}

The initial efforts have been directed toward two main objectives: (1) construction of a diamond anvil cell that could withstand the high-temperature annealing and (2) development of new techniques to measure free carrier concentration in extremely small samples.

We have fabricated a small diameter diamond anvil cell that could be annealed in an evacuated quartz tube. We anticipated that such a design would allow us to carry out post-annealing optical measurements without removing the samples from the cell. We have performed several annealing tests with annealing temperatures in the range 800 to $1100 \mathrm{~K}$ and with pressures up to $8 \mathrm{GPa}$. We found that the reduced mechanical strength of the small size cells had a detrimental effect on the capability of the cells to hold the high hydrostatic pressure. Also in some cases an inhomogenous thermal expansion was responsible for shattering of the diamond anvils. Presently, in collaboration with the Department of Geophysics at UC Santa Cruz, we are conducting experiments on the annealing of GaAs in a larger diamond anvil cell in which only the central part of the cell is heated. It has been demonstrated that the annealing at temperatures exceeding $1200 \mathrm{~K}$ can be performed in such cells with only a modest reduction of the pressure.

We have developed a method to determine the free electron concentration in very small $\mathrm{GaAs}$ samples. The method is based on the measurements of capacitance-voltage characteristics. Preparation of good quality Schottky contacts with low leakage currents to the samples with the surface area of less than $10^{-3} \mathrm{~cm}^{2}$ was the main problem in accomplishing these measurements. As is shown in Figure 8, we were able to fabricate very small diodes with very good $C-V$ characteristics, enabling determination of the free electron concentration with a $10 \%$ precision. This technique provides a basis for the evaluation of the GaAs samples processed in the diamond anvil cell. In the case of $\mathrm{ZnSe}$ we measure Raman spectra of holeplasmon coupled modes. An analysis of the lineshape and energy shifts provide quantitative information on free hole concentration in the valence band.

During the last several months we were able to identify the diamond anvil cell design most suitable for high-pressure, high-temperature annealing of semiconductor materials. We have also developed specific characterization techniques for a quantitative evaluation of the properties of the processed materials. 


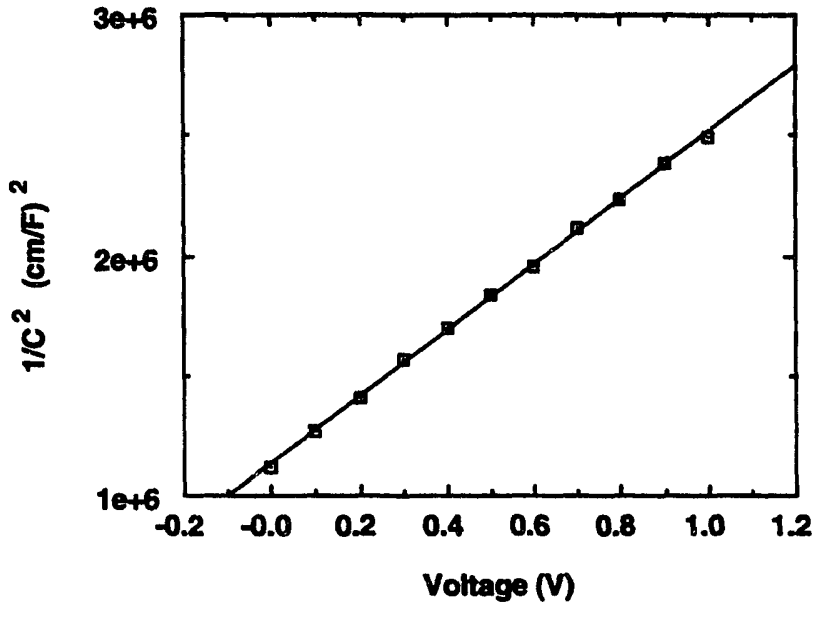

Fig 8. The free carrier concentration in our samples can be obtained from the slope of the capacitance-voltage plot as shown. In order to accomplish these measurements, techniques were deoeloped to prepare good quality Schottky contacts with low leakage currents on samples with a surface area of less than $10^{-3} \mathrm{~cm}^{2}$.

This funding has helped to establish material basis and scientific expertise necessary to perform experiments, currently in progress, that will provide the final test of our original hypothesis.

\section{Publications}

W. Walukiewicz and J. A. Wolk "Effect of Hydrostatic Pressure on Electrical Activity of Shallow Impurities in Compound Semiconductors," presented at the 1992 APS March Meeting, Bull. Amer. Phys. Soc. 37, 195 (1992).

\section{Scanning Tunneling Microscopy of Heterostructures and Interfaces in Cross Section}

Principal Investigators: Eicke R. Weber and Miquel B. Salmeron

Funding: $\$ 104,300$

\section{Project Description}

The goal of this project is to spatially resolve the geometric and electronic structure of interfaces in epitaxial systems, and to detect defects in thin film layers and at interfaces. This approach is expected to have a major long-term impact in the understanding of the electronic structure of heterostructures, epitaxy, and defects at interfaces and in thin films. Particularly, the questions of the conduction mechanism in lowtemperature grown GaAs (LT-GaAs) and the mechanism of Schottky barrier formation of metal/GaAs Schottky contacts will be addressed.

High-resolution scanning tunneling microscopy (STM) in an UHV-chamber allows observation at atomic resolution of the top atomic layer of a sample prepared in situ. Moreover, the possibility to obtain the I/V characteristics of any atomic location makes the STM a spectroscopic instrument. However, the general application of STMs uses "plan-view" geometry for surface science research, probing only the top few layers, which is not very useful for the study of thin film heterostructures. For such structures it will be extremely powerful to study cross sections of thin films and interfaces created by in situ cleavage in UHV. This will allow to directly image and analyze with atomic resolution the electronic structure of thin films such as quantum confined structures or low-temperature grown compound semiconductors and interfaces such as metal/semiconductor interfaces or weak links (SIS or SNS type) in superconductors. In the last decade, cross-sectional transmission electron microscopy (TEM) has brought a breakthrough in the study of the atomic structure of heterostructures; it is expected that this cross-sectional approach in STM and later in the related atomic force microscopy (AFM) will open up new horizons as well.

Central to this project is the development of a new kind of thin film and interface analysis technique through STM of thin film structures studied in cross section after in situ cleavage in an UHV-chamber. This approach makes accessible the full power of scanning tunneling microscopy for investigations of heterostructures such as quantum wells, strained layer superlattices, semi-insulating GaAs epilayers, metalsemiconductor and metal/superconductor junctions. This project complements in an ideal way the ongoing structural studies of heterostructures by cross-sectional TEM in NCEM, as it allows to resolve individual atom positions near interfaces and to obtain information of the electronic structure in atomic resolution.

\section{Accomplishments}

The construction of a UHV-compatible STM device with controlled $x$ - and $y$-axis translation of the sample after UHV-cleavage has been successfully fabricated. A UHV-chamber was designed and constructed to house the STM head and allow for sample exchange 
through a load-lock and in situ sample cleavage. We selected a rather compact chamber construction to minimize pump-down and back-out times. The design can be readily upgraded with a low-temperature stage for in situ studies to below $20 \mathrm{~K}$. Optical access is also available for in situ illumination and for the detection of luminescence excited by the carrier injection from the STM tip. The STM design is based on the so-called "Beetle-STM." It consists essentially out of four identical piezo crystals, three holding the sample plate and the fourth the STM tip. The home-built electronic sample manipulator can remotely control several millimeters of $x$ - and $y$-translation from outside the UHV chamber. The z-approach can be realized by rotation of a specially shaped sample holder plate or with the $x$-translation if the cleavage surface is mounted at an angle to the plate. In the latter case, the $y$-translation can be used to approach the interface or thin film of interest. The STM head was mounted in this chamber, and it has been tested with atomic resolution on cleaved GaAs (110) surface in cross section, Figure 9. The z-resolution has been determined to be better than $0.1 \AA$. The first results of STM of UHV-cleaved heterostructures viewed in cross section were recently obtained for metal/ semiconductor contacts and semiconductor heterostructures.
In Figure 10a, a first STM image of a semiconductor heterostructure of alternating layers of n-type GaAs and AlGaAs is shown, with period of $140 \mathrm{~nm}$. This image does not yet show atomic resolution, but it is interesting to note that the apparent corrugation probed by the STM tip in the constant-tunnel current mode is $20 \AA$. Tunneling out of the sample shows a much weaker corrugation if the tip voltage is inversed, i.e., for tunneling out of the heterostructure. The physical mechanism for this warrants further study.

The investigation of metal/semiconductor structures utilizes the crystallography of the zinc-blende lattice with $\{110\}-$ cleavage planes, two of which are mutually perpendicular. This also has allowed investigation of UHVclean metal/semiconductor interfaces deposited in UHV after in situ cleavage of a semiconductor crystal. An image of a metal/ semiconductor heterostructure cleaved in UHV was obtained, Figure 10b. This figure shows clearly a perfect cleavage of the GaAs up to the interface, but a very poor propagation of the cleavage into the metal, as expected. However, the interest of this work lies in studying the semiconductor near the interface, so that this first result demonstrates the feasibility of this approach. It might be necessary to select sample areas for this type of study where the metal surface is

\section{Cross-Sectional STM Image of Filled States on GaAs(110) Surface}
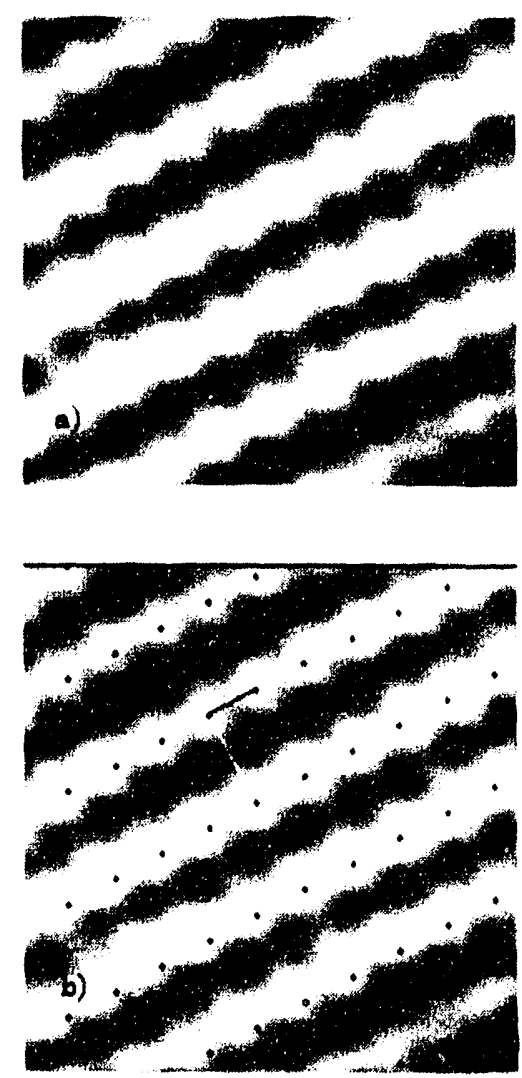

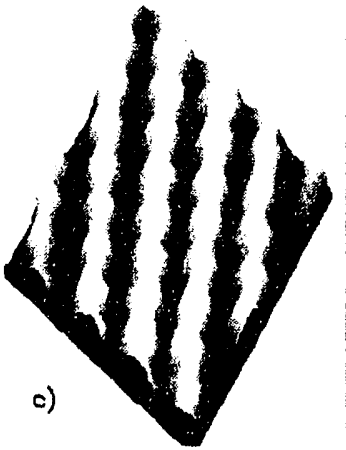

c) 3d Viow lmage

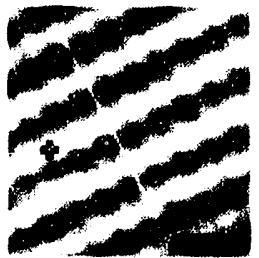

d) Cursor position

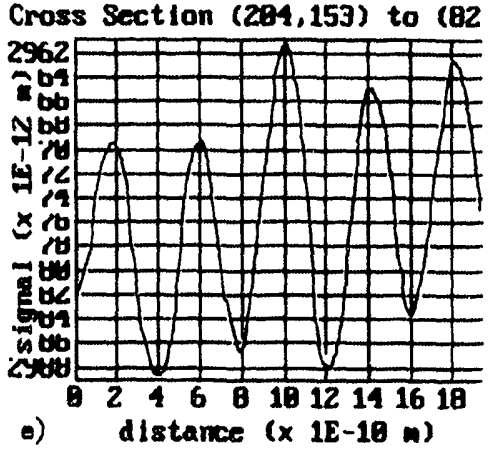

-) Cursor plot along $\langle 100\rangle$

f) Cursor plot along $\langle 110\rangle$

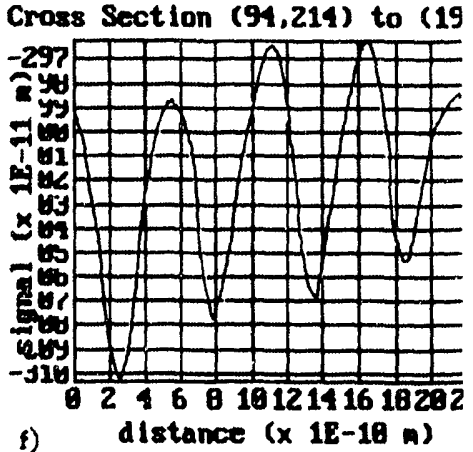


Blas Voltage Dependent Image of Semiconductor Heterostructure By Cross-Sectional Scanning Tunneling Microscope
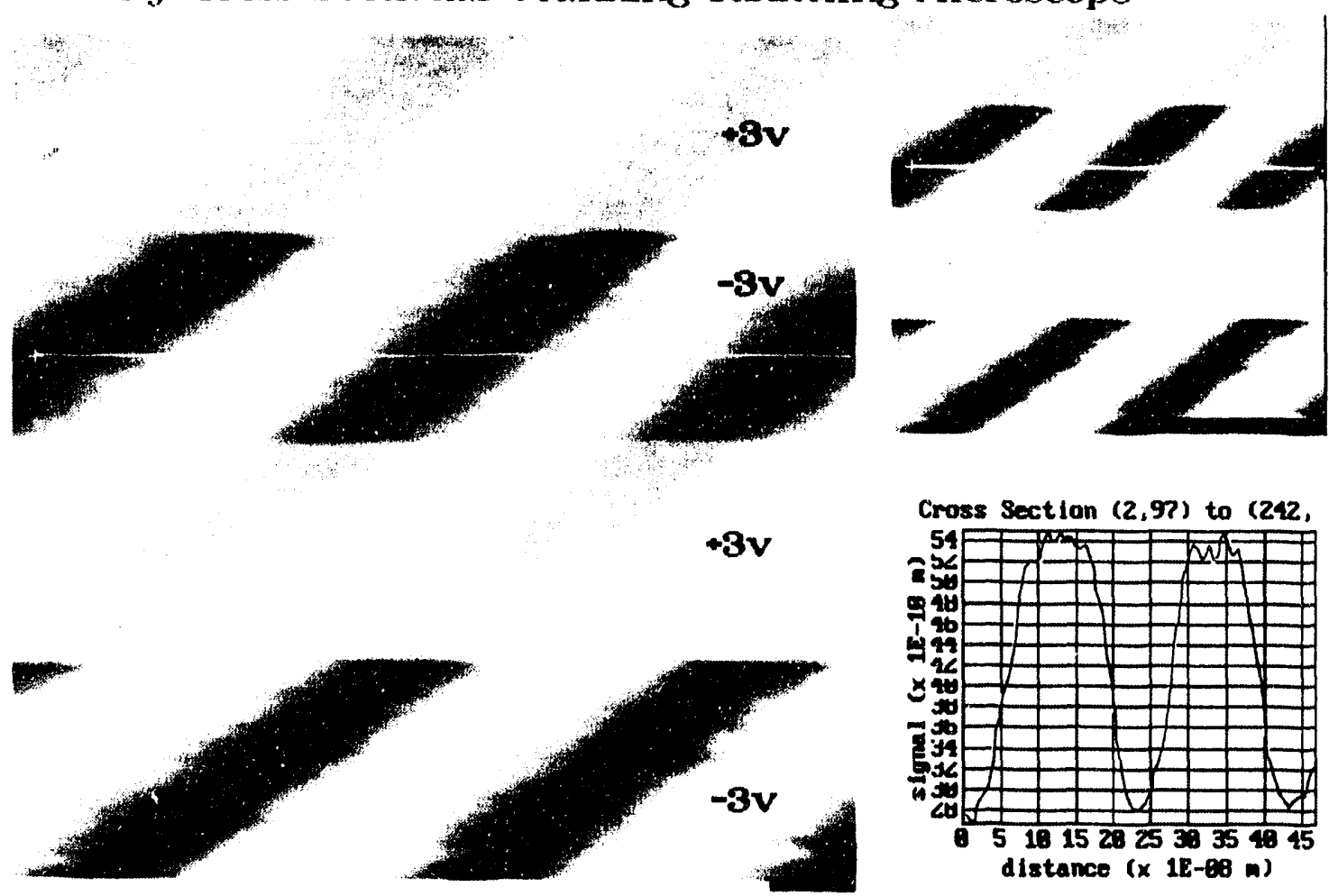

Fig. 10a. Bias voltage dependent image of semiconductor heterostructure by cross-sectional scanning tunneling microscope.

\section{Cross-Sectional STM Image of $\mathrm{Al} / \mathrm{n}$-GaAs Interface}

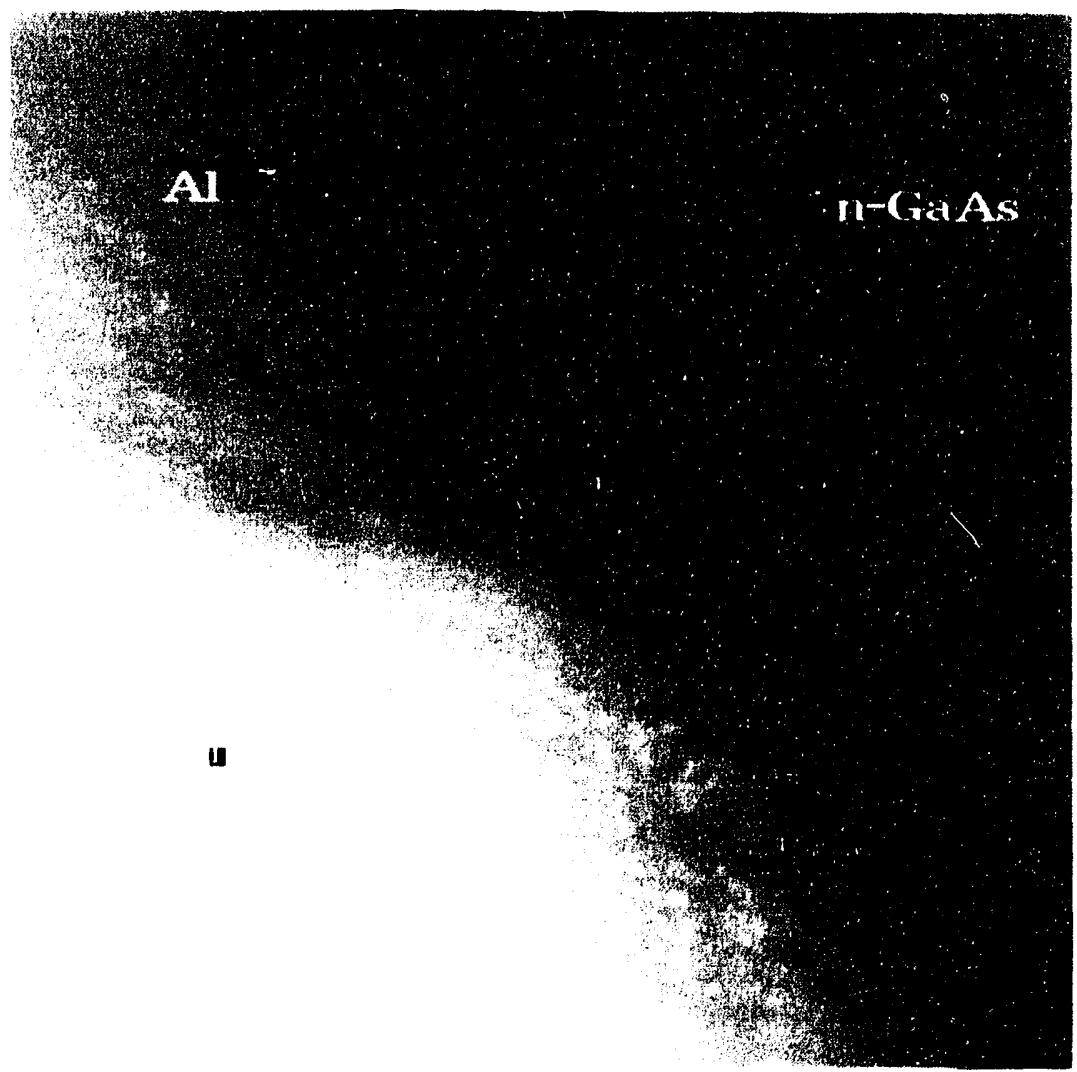

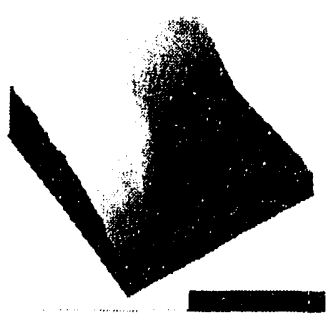

3D View
* $1,2,3$ and 4 points are the locations $1 / \mathrm{V}$ measurement were taker.

Fig. 10b. Cross-sectional STM image of $A l / n-G a A s$ interface. 
depressed as compared to the semiconductor in order to allow for tip approach right up to the interface. I-V analysis at various points across the interface shows the change of electronic properties from metallic to semiconducting. This is a most promising approach to answering the century-old question as to the mechanism of Schottky barrier formation at metal/ semiconductor interfaces. It makes it possible to topologically map the atomic positions near the interface, to distinguish the defects in the compound semiconductor near the interface in order to identify extrinsic interface states, and to use the unique capability of I-V mapping to study the local electronic structure of intrinsic and extrinsic interface states and the band bending near the interface with atomic resolution. This work should lead to significant progress in the understanding of the mechanism of Fermi-level pinning at metal/ semiconductor junctions. The structures found at interfaces can be compared with surface defects created by techniques such as ion bombardment, non-stoichiometric bulk-growth conditions (i.e., Ga-rich or As-rich), high concentration metal-impurity doping, exposure to controlled levels of contamination or deposition of submonolayer coverages of metals. From these studies, the electronic and structural characteristics of point defects (such as vacancies and antisites), small defect clusters, and dislocations will be determined.

\section{Publications}

J.F. Zheng, F. Ogletree, M. Salmeron, and E.R. Weber, "The Development of a Cross-Sectional Scanning Tunneling Microscope for the Study of Semiconductor Heterostructures," presented at the XV Surface and Interface Research Meeting, Berkeley, CA, June, 1992.

\section{Solids and Clusters Based on Fullerenes}

Principal Investigators: Alex Zettl and Marvin L. Cohen

Funding: $\$ 198,400$

\section{Project Description}

The goal of this project has been to establish a pilot program for the experimental and theoretical investigation of fullerene-based materials. Fullerenes represent new forms of carbon, such as spherical molecules $\left(C_{60}\right)$, elongated molecules $\left(C_{70}\right)$, and extended tubes. These novel materials display unusual electronic and mechanical properties. The small diameter "nanotubes," which may be conducting depending on the tube diameter, are an example of an extremely elongated fullerene. $C_{60}$ doped with alkali metal can form superconducting compounds with transition temperatures exceeding $30 \mathrm{~K}$. The superconductivity mechanism has not yet been established.

Many of the fundamental interactions and characteristic parameters for this new class of materials are not yet known. High-quality single crystals and isolated bulk materials are desired to perform characterization experiments. It is also desired to attempt synthesis of novel structures of fullerene-based materials, either insulating or conducting.

As a starting point this project will establish a materials synthesis and characterization laboratory dedicated to fullerenes. Since alkali-doped fullerenes are extremely reactive, appropriate facilities need to be constructed. Transport and mechanical measurements will be carried out and analyzed using appropriate theoretical models.

\section{Accomplishments}

A materials synthesis laboratory for the generation and purification of different fullerenes has been established. Thin films, powders, and single crystals of various fullerenes (including $C_{60}$ ) have been prepared. The crystals have been characterized by structural ( $x$-ray diffraction), thermodynamic (specific heat), and mechanical (Young's modulus) measurements. Rubidium and potassium doping of the single crystals has been carried out, and these samples have formed the basis of several transport studies.

Elasticity measurements on pure $\mathrm{C}_{60}$ crystals have established an harmonicity in the interball potentials, and have determined the characteristic Debye temperature. Specific heat and electricity measurements have investigated the ball-rotation ordering near $265 \mathrm{~K}$.

The intrinsic temperature dependent resistivity of $\mathrm{K}_{3} \mathrm{C}_{60}$ crystals has been determined. The behavior is metal-like (in contrast to transport data reported by other groups on thin films). The resistivity has been used to test various models of electron-electron and electron-phonon scattering, and superconductivity mechanisms. In an electron-phonon model, both low frequency interball and high-frequency intraball modes are required to account for the resistivity. Magnetotransport measurements have determined 
fundamental parameters such as the superconducting coherence length, upper critical field, mean-free path, penetration depth, scattering time, and intrinsic resistivity. Pure 3-dimensional paraconductivity fluctuations have been observed in $\mathrm{K}_{3} \mathrm{C}_{60}$ and $\mathrm{Rb}_{3} \mathrm{C}_{60}$. These measurements have provided tests of scaling theories. An isotope-effect project has been initiated and is presently underway.

\section{Publications}

X-D. Xiang, J.G Hou, G. Briceno, W.A. Vareka, R. Mostovoy, A. Zettl, V.H. Crespi, and M.L. Cohen, "Synthesis and Electronic Transport of Single Crystal $\mathrm{K}_{3} \mathrm{C}_{60}$," Science 256, 1190 (1992).

V.H. Crespi, J.G. Hou, X.-D. Xiang, M.L. Cohen, and A. Zettl, "Electron-Scattering Mechanisms in SingleCrystal K ${ }_{3} \mathrm{C}_{60}$," Phys. Rev. B46, 12064 (1992).

M. Chung, Y. Wang, J.W. Brill, X.-D. Xiang, R. Mostovoy, J.G. Hou, and A. Zettl, "Ac Calorimetry of C60 Single Crystals," Phys. Rev. B45, 13831 (1992).

Y. Jin, J. Cheng, M. Varma-Nair, G. Liang, Y. Fu, B. Wunderlich, X.-D. Xiang, R. Mostovoy, and A. Zettl,
Thermodynamic characterization of $\mathrm{C}_{60}$ by differential scanning calorimetry, J. Phys. Chem. 96, 5151 (1992).

S. Hoen, N.G. Chopra, X.-D. Xiang, R. Mostovoy, J.G. Hou, W.A. Vareka, and A. Zettl, "Elastic Properties of a van der Waals Solid: C60," Phys. Rev. B46, 12737 (1992).

X.-D. Xiang, J.G. Hou, V.H. Crespi, A. Zettl, and M.L. Cohen, "Three-Dimensional Fluctuation Conductivity in Single Crystal $\mathrm{K}_{3} \mathrm{C}_{60}$ and $\mathrm{Rb}_{3} \mathrm{C}_{60}$," Nature, in press.

J. G. Hou, V.H. Crespi, X.-D. Xiang, W.A. Vareka, G. Briceno, A. Zettl, and M.L. Cohen, "Determination of Superconducting and Normal State Parameters of Single Crystal $\mathrm{K}_{3} \mathrm{C}_{60}$," submitted to Solid State Commun.

M.L. Cohen and V.H. Crespi, "Theory of Electronic and Superconducting Properties of Fullerenes," in Buckminsterfullerene and Related Carbon Clusters, eds. Billips and Ciufolini, in press.

M.L. Cohen, "Theory of Normal and Superconducting Properties of Fullerene-Based Solids, in Nanostructures, in press.

S.L. Dexheimer, D.M. Mittleman, R.W. Schoenlein, W. Vareka, X.-D. Xiang, A. Zettl, and C.V. Shank, in Ultrafast Phynonena VIII, A. Miguo and J.L. Martin, eds., Springer-Verlag, in press. 


\section{Nuclear Science Division}

\section{Laser Trapping of Neutral Atoms}

Principal Investigator: Stuart Freedman

Funding: $\$ 99,600$

\section{Project Description}

The goal of this work is to develop neutral atom manipulation techniques that will be applied to fundamental beta-decay experiments. We completed a major part of the initial construction: (1) An off-line facility was setup for developing the necessary techniques of trapping and cooling to be used on the online experiments. (2) A laser system was constructed near the 88-Inch Cyclotron to expedite the on-line experiments. In addition we began to develop techniques for expediting the first experiment, a study of the beta asymmetry of ${ }^{21} \mathrm{Na}$ as a test of the $\mathrm{V}-\mathrm{A}$ structure of the weak interaction. The accomplishments follow after we briefly describe the methods and procedures to be used in the ${ }^{21} \mathrm{Na}$ experiment.

Figure 1 shows the basic scheme for the first on-line experiment with ${ }^{21} \mathrm{Na}$ whose half-life is about $23 \mathrm{sec}$. A $25 \mathrm{MeV}, 100 \mathrm{nA}$ beam from the LBL 88-Inch Cyclotron interacts with metallic magnesium chips con-

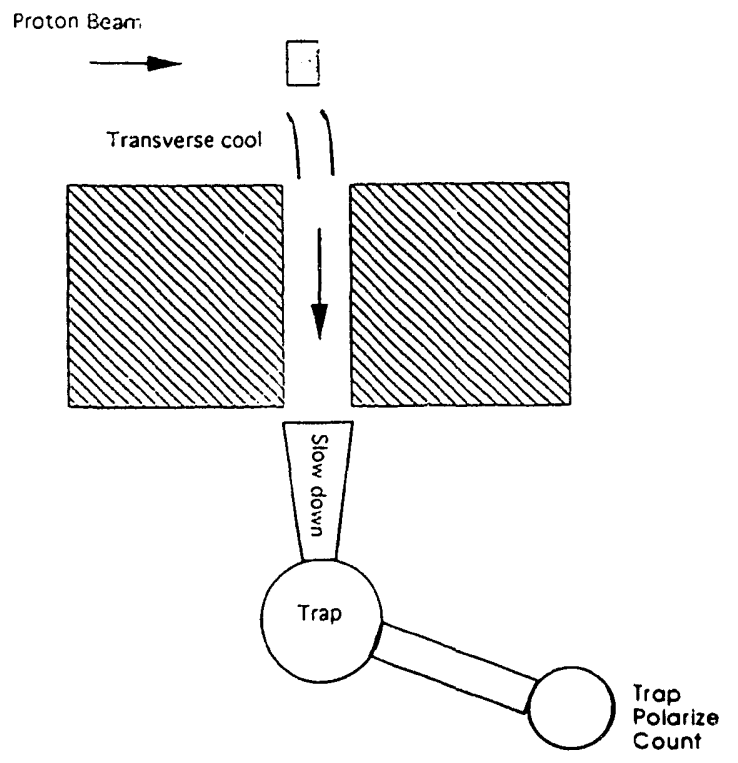

Fig. 1. Schematic diagram of the experimental scheme for beta decay experiments with trapped atoms. tained in a small atomic beam oven. Radioactive ${ }^{21} \mathrm{Na}$ atoms are produced via the ${ }^{24} \mathrm{Mg}(\mathrm{p}, \alpha)^{21} \mathrm{Na}$ reaction, and the resulting atomic beam moves through hole in the shielding wall to a low-background counting area. The trarisverse momentum distribution of the beam is cooled with laser light to enhance the on-axis flux by sending the beam down the axis of a linearly varying solenoid magnetic field of a tapered slowing magnet. A counter propagating laser beam brings the atomic beam to rest at the end of the magnet, and the atoms are collected in a magneto-optical trap. The trapped atoms are moved to a nearby counting area where they are held in a second magneto optical trap (the possibility of using a pure magnetic trap instead is still under consideration).

The second magneto-optical trapped is cycled on and off at the millisecond time scale. During the off periods the atoms are polarized by optical pumping, and the decay positrons are detected in plastic scintillation counters. We estimate that this procedure will allow us to measure the beta-decay asymmetry parameter to the level of a percent.

Once the beta-asymmetry parameter is measured we will combine it with measurements of the decay lifetime and the value of the vector coupling constant from other nuclear beta decays. After accounting for the possibility of forbidden contributions to the beta decay of ${ }^{21} \mathrm{Na}$, this result will provide constraints on possible right-handed components to the fundamental weak interactions.

\section{Accomplishments}

An off-line laser laboratory was set up in Birge Hall. We have installed an argon-ion laser pumped dye laser and optics appropriate for trapping experiments with stable ${ }^{23} \mathrm{Na}$. In the initial series of experiments we studied the parameters of the magneto-optical traps. Sodium was collected in the center region of a magnetic field generated by two $3-\mathrm{cm}$ diameter coils that produced opposing magnetic fields. Atoms are captured from the low-energy tail of a very lowpressure vapor contained in a cell at room temperature. About $10^{8}$ atoms were collected in an approximately $2-\mathrm{mm}$ diameter region. The quantity of atoms captured would be completely adequate for the planned experiments. In the course of this work we 
discovered what appears to be a new trapping mechanism for scidium that is caused by transition between the $F=1$ hyperfine levels of the ground and first excited states of ${ }^{23} \mathrm{Na}$. Experiments are underway to elucidate the properties of the new trapping mechanism that seems to allow larger densities of trapped atoms. Construction of a high-vacuum atomic beams machine is about half done. This apparatus will allow us to test our procedures for trapping and manipulating atoms captured from an atomic beam.

An intense beam of ${ }^{21} \mathrm{Na}$ was produced in experiments at the 88-Inch Cyclotron. The radioactive sodium was produced with the ${ }^{24} \mathrm{Mg}(\mathrm{p}, \alpha)^{21} \mathrm{Na}$ reaction using an 25 $\mathrm{MeV}$ proton beam. Ordinary magnesium in the form of small chips was contained in a small stainless steel oven that was attached to a copper heat sink. The measured beam was $4 \times 10^{7}$ atoms per second. The beam is already sufficient for the planned experiment with trapped ${ }^{21} \mathrm{Na}$. The most recent version of the atomic beam system operates reliably for about two days. We intend to further increase the lifetime of the oven and to replace the stainless steel oven with a tantalum one to avoid activation, allowing us to reuse ovens.

A laser laboratory was set up at Building 88. Sodium resonance light is brought to the experimental area through $50 \mathrm{~m}$ of fiber optics guide. The first cooling

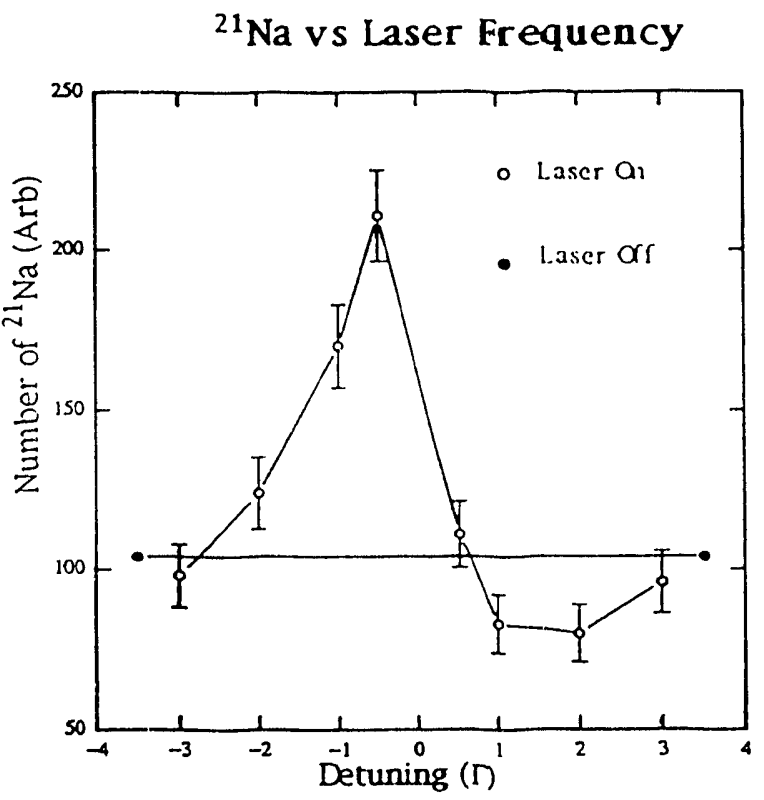

Fig. 2. First signal of transverse cooling. The on axis flux of an atomic beam of ${ }^{21} \mathrm{Na}$ is enhanced by laser cooling. The horizontal scale is in units of the natural line width, about $10 \mathrm{MHz}$. experiments with radioactive atoms were carried out with a simple mirror system. In the initial test a signal for transverse cooling was observed as shown in Figure 2. As far as we know this is the first time radioactive atoms have been cooled. The present setup will be used in an upcoming run to measure the lifetime and decay branches in ${ }^{23} \mathrm{Na}$ decay.

A system of curved mirrors was designed and constructed. The curved mirror will allow us to enhance the efficiency of transverse cooling, providing another factor of ten improvement of the on-axis beam flux of radioactive atoms.

A tapered solenoid magnet was designed and constructed. This magnet will allow us to proceed with the next major step: bringing the atoms to rest inside a magneto-optical trap.

\section{New Concepts for High-Multiplicity Tracking Detectors}

Principal Investigator: John W. Harris

Funding: $\$ 201,800$

\section{Project Description}

The high-track densities expected at future highenergy, nuclear, and particle physics accelerators demand precision tracking detectors and new techniques. This project addresses four aspects of potential high-multiplicity tracking detectors that are novel and that require significant development for implementation as detectors: (1) simulations, development, and prototyping of silicon vertex tracking (SVT) detectors suitable for the high-multiplicity tracking environment near the interaction region; (2) prototyping and testing a radial-drift Time Projection Chamber (TPC) for use as forward angle multiplicity counters; (3) computer simulations for developing algorithms for finding and measuring jets using tracking detectors and electromagnetic calorimetry (without hadronic calorimetry); and (4) development and testing of various pad plane readouts in order to increase spatial resolution in TPCs.

\section{Accomplishments}

Silicon drift detectors with active area $1 \times 1.8 \mathrm{~cm}^{2}$ were produced in collaboration with Brookhaven National Laboratory and tested for performance. The detectors were tested in the laboratory using $8 \mathrm{~ns}$ pulses of 1064 
nm light transported via light fibers from a Nd:Yag laser. TPC electronics were modified for use in reading out the silicon drift detectors and a custom data acquisition system was developed. In order to determine the two track resolution of the detectors, multiple light spots of varying separation were illuminated on the detector using the laser and fiber optics. The drift characteristics were simulated and found to agree well with the measurements of the devices.

The capability of the silicon drift detectors to measure energy loss in the $1 / \beta^{2}$ region was investigated by experiment and simulations. Tests of a silicon drift detector were made with $300 \mathrm{MeV} / \mathrm{c}$ pions at the TRIUMF accelerator facility. A signal-to-noise ratio of 55 to 1 was measured. From simulations it was determined that a maximum energy loss resolution of $15 \%$ was possible. Comparisons of the data with these simulations show that each type of charged particle, with the exception of muons, is expected to be distinguishable from the others using the silicon drift detector if the momentum of the particle is $p<m_{0} c$, where $m_{0}$ is the mass of the particle and $c$ the speed of light.

A radial drift TPC prototype was designed and constructed. The principle of operation of the radial TPC is to drift ionization from tracks in a radially outward direction in order to magnify the distance between tracks and improve two-track resolution. The prototype is a 45 degree wedge with a drift length of 1 foot. The prototype was tested in beam at the AGS accelerator at Brookhaven National Laboratory in conjunction with conventional tracking detectors. The beam particles were identified and measured in the prototype at positions corresponding to those expected from projections of the particle trajectories from the associated tracking detectors. Thus, the radial concept was shown to work with minor distortions on the drifting due to the fields. The radial prototype has been modified and will be tested with an electron source prior to subsequent detailed testing in beam to quantify its performance characteristics. In particular, the distortions are being studied and the possibility of implementing hardware corrections in the electric drift field are being pursued.

In order to determine the feasibility of performing measurements of jets and high- $p_{t}$ particles by using a combination of tracking and electromagnetic (EM) calorimetry, thereby eliminating costly hadronic calorimetry, computer simulations were performed to investigate the two techniques, [EM + hadronic] and [EM + tracking]. Jet events were simulated in Pp collisions using a physics eveni generator HIING, decaying all produced particles with lifetimes shorter than the $\mathrm{K}_{\mathrm{L}}^{\circ}$ and considering the energy produced in the form of photons, charged particles and hadrons (both charged and neutral). An implementation of the CDF jet-finding algorithm was then applied to the [EM + hadronic] and [EM + tracking] data sets. The energy resolution for $\mathrm{p}_{t}=40 \mathrm{GeV} / \mathrm{c}$ jets in [EM + tracking] is $17 \%$, slightly worse than the $14.5 \%$ for [EM + hadronic]. For $\mathrm{p}_{t}=30 \mathrm{GeV} / \mathrm{c}$ jets the two methods yield the same resolution and for $\mathrm{p}_{t}=20 \mathrm{GeV} / \mathrm{c}$ jets the [EM + tracking] resolution is $32 \%$ compared to $40 \%$ for [EM + hadronic]. This trend was found to be a result of the rapidly decreasing hadronic calorimeter resolution as the jet and particle energies decrease.

Studies of the ability of the EM calorimeter to deliver a trigger for events containing jets or several high-pt particles have begun with the simulations of pp events as described above. Calculations were performed for two possible triggers, one based on $E_{t}$ within the EM calorimeter and a second based on a single high- $\mathrm{p}_{t} \pi^{0}$. The efficiencies of these triggers were derived as a function of jet $p_{t}$. As an example, for $30-\mathrm{GeV} / \mathrm{c}$ jets the optimum electromagnetic $E_{t}$ trigger used a 5-GeV EM energy threshold and was found to be $94 \%$ efficient. For comparison, the best single high- $p_{t} \pi^{0}$ trigger used a $2-\mathrm{GeV}$ threshold and was $82 \%$ efficient.

In general, it was determined that the technique of using a combination of tracking and EM calorimetry to trigger on and measure jets and high-pt particles is feasible with some loss of resolution and a large cost saving due to elimination of the hadronic calorimetry.

In an attempt to improve the voltage gain stability of TPCs, the effects of eliminating the field wires from a conventional TPC were measured. An existing chamber was divided into two sections, one with field wires and one without. A movable electron point source was developed to facilitate measuring the pad response function (pad signal amplitude as function of the electron source position relative to the pad). The point electron source was constructed from a half transparent photo cathode illuminated by a focused UV beam conducted by fiber optics from a nitrogen laser. The results of these measurements were surprising; the pad response function is practically indistinguishable for the two cases. Thus, it was concluded that the field wires can be eliminated from a TPC without compromising performance.

Additional measurements have been carried out comparing the avalanche gains to determine the maximum possible gain in each case. These data are presently being analyzed.

To determine the spatial resolution and operating 
characteristics of a smaller pad (higher resolution) design, a prototype chamber with small pads $(2.85 \mathrm{~mm}$ by $11.5 \mathrm{~mm}$ ) has been constructed and tested using $x-$ ray sources. The performance of this chamber is being fully characterized using the point electron source.

\section{Publications}

G. Odyniec, "The Silicon Vertex Tracker for STAR," presented at the Workshop on Front End Electronics for Silicon Detectors at Future High Luminosity Colliders, Mammoth Hot Springs, Yellowstone National Park, Wyoming, September 17-19, 1992.

C. Naudet, "Laboratory Test Results of Silicon Drift Detectors," presented at the Workshop on Front End Electronics for Silicon Detectors at Future High Luminosity Colliders, Mammoth Hot Springs, Yellowstone National Park, Wyoming, September 1719, 1992.

\section{New Concepts in Isospin Studies}

Principal Investigator: J. Michael Nitschke

Funding: $\$ 63,400$

\section{Project Description}

The purpose of this project is to evaluate the scientific requirements for an IsoSpin Laboratory (ISL). The ISL will facilitate nuclear reactions with beams of radioactive in addition to stable ions and thus expand nuclear physics research in the new dimension of isospin. This is achieved by coupling two accelerators: a light ion machine for the production of the radioactive nuclei and a heavy-ion accelerator for post-acceleration. A national facility of this kind is presently under discussion within the nuclear physics community; it is expected to be sited at a major laboratory in North America before the end of this decade. Several questions need to be addressed before definitive proposals can be submitted to the ISL Steering Committee, NSAC, and/or DOE:

1. What is the optimum method for producing highintensity, high-energy, light-ion beams?

2. What are the expected activity inventories, radiation levels, and shielding requirements?
3. What are the optimum targets and ion sources for on-line operation?

4. What is the optimum method for accelerating low velocity, low charge-to-mass-ratio ions?

The goal of this project was to provide answers to the above questions by drawing mainly on in-house expertise and in some cases outside consultants.

\section{Accomplishments}

An important issue is to find a cost-effective way to produce the primary light-ion beams that are needed for the first stage of the ISL. After consultation with several accelerator experts, it has become clear that a cyclotron could produce these ions in a more economical way than a LINAC. Our present conclusion is, therefore, that an $\mathrm{H}^{+}$cyclotron with fixed energy (600 $\mathrm{MeV}$ ) would be most appropriate for an LBL-based ISL. In addition to lower capital cost, the operating cost of the cyclotron would be a fraction of that of a proton LINAC like LAMPF. Another important benefit would be that the primary beam accelerator would be dedicated $100 \%$ of its time to radioactive beam production. A conceptual design study has been carried out and resulted in a compact isochronous cyclotron with a diameter of $\sim 10 \mathrm{~m}$ and a peak field of $2 \mathrm{~T}$. It has high extraction efficiency ( $>99 \%)$ and is injected from an external ion source. One of its unique features would be the magnet design, with 4 sectors at the center and a transition to 8 sectors part way out in radius.

The second task was to carry out calculations of the expected activity inventories, radiation levels, and shielding requirements for the ISL. The principal radiation problems are associated with neutrons produced in the interaction of the high-energy proton beam with various targets. High-energy

( $\mathrm{E}>20 \mathrm{MeV}$ ) intranuclear cascade neutrons are the most difficult to shield against and will require about $10 \mathrm{~m}$ of ordinary concrete or $5 \mathrm{~m}$ of steel in the forward direction. These results were reported at an ISL Technical Workshop held at ORNL this year and in a report by our consultant.

The most important parameter of the ISL will be the distribution of the radioactive beam intensities as a function of the mass- and element numbers of the accelerated nuclei. Intensity calculations have been carried out using the Silberberg-Tsao cross section formalism. The energy deposition in potential ISL targets was evaluated using the intranuclear cascade codes LAHET and FLUKA combined with the finite element analysis code ANSYS. Using these computer 
simulations we designed a target that will support a maximum beam power of $40 \mathrm{~kW}$, which meets the design specifications of the ISL. To check these calculations a target test stand is under construction where full-scale ISL targets can be tested with electron beams at a maximum power level of $50 \mathrm{~kW}$.

The final part of the project addressed the problem of finding the best method for accelerating the lowvelocity, low-charge-to-mass-ratio radioactive ions to energies sufficient for nuclear interactions. Specifically, whether it is possible, in an initial phase, to accelerate the ions in the SuperHILAC. This would result in a significant initial cost savings. The coupling of the ion sources to the SuperHILAC could be accomplished with RFQs and/or low- $\beta$ structures. Only a very preliminary study was performed, since it turned out that the acceleration of low-velocity, low-chargeto-mass-ratio heavy ions is technically difficult. An international working group is being formed to deal with this challenge.
Publications

D.J. Clark, Proceedings of the XIIIth Intl. Conf. on Cyclotrons and their Applications, Vancouver, Canada, 1992, to be published.

D.J. Clark, Proceedings of the Workshop on the Production and Use of Intense Radioactive Beams at the IsoSpin

Laboratory, Oak Ridge, TN, 1992, edited by J.D. Garrett, to be published.

R.J. Donahue, Proceedings of the Workshop on the Production and Use of Intense Radioactive Beams at the IsoSpin Laboratory, Oak Ridge, TN, 1992, edited by J.D. Garrett, to be published.

J.M. Nitschke, Proceedings of the Workshop on the Production and Use of Intense Radioactive Beams at the IsoSpin Laboratory, Oak Ridge, TN, 1992, edited by J.D. Garrett, to be published. 


\section{Physics Division}

\section{Data Acquisition Electronics}

Principal Investigators: Michael Levi and William Greiman

\section{Funding: \$ 87,700 (FY 92)} $\$ 107,000$ (FY 91)

\section{Project Description}

The purpose of this program was to effect advances in the state-of-the-art of high-bandwidth data-acquisition systems that will be essential for the successful operation of particle physics detectors envisioned in future high-luminosity experiments later this decade.

The detectors envisioned for future colliders will require data-acquisition systems capable of handling data rates one hundred to one thousand times higher than the current generation of experiments. An average data rate of $10^{9}$ to $10^{10}$ bits per second will be the expected norm for these future experiments. Data collection electronics will funnel data from a moderate number of high-speed links. The data-acquisition system must provide minimal deadtime and a substantial reduction in data rate, through the application of sophisticated event processing provided by large processor farms and special-purpose processors. An event builder assembles the data from the various detector elements into a complete event record containing all data associated with a particular event.

\section{Accomplishments}

A scalable parallel data-acquisition system architecture based on a commercial gigabit data switch has been designed, see Figure 1 . This switch supports the ANSI standard Fiber Channel protocol. Complete nonblocking interconnection is provided and performance scales linearly with the number of nodes. The parallel event builder based on this switch is shown in Figure 2. The key features of this design are:

- Readout synchronization in the trigger system and front-end electronics. This allows a simple data flow architecture to be implemented.

- Decoupling real time event rates by buffering and large message size. This reduces the transaction rate in event builder nodes to less than 100 messages/second.

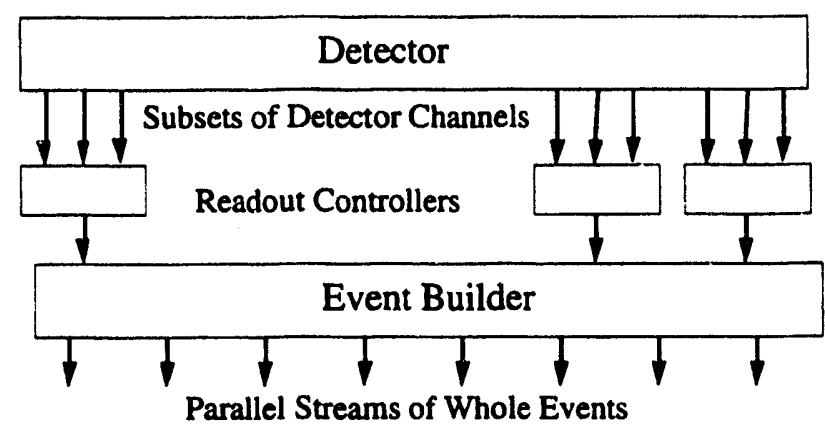

Fig. 1. Architecture of the primary flow of data from the detector to processing and recording elements in the highperformance data-acquisition system.

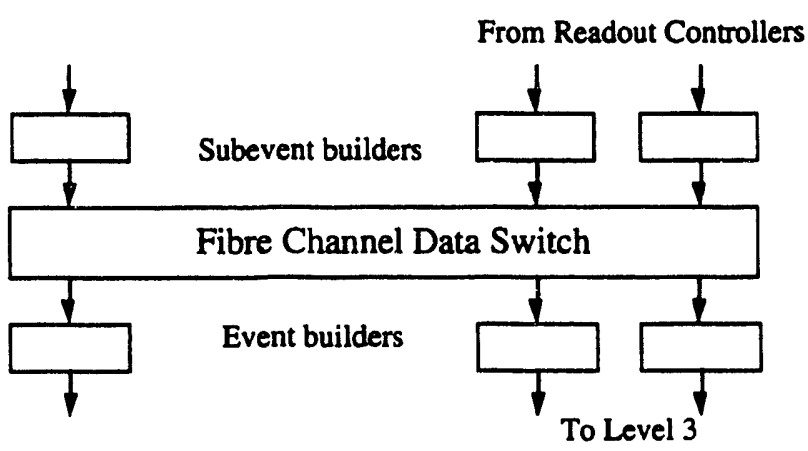

Fig. 2. A scalable parallel event builder. This architecture has three stages, subevent builders, data switch, and full event builders. All data paths are assumed to be 1 Gigabit/ sec Fibre Channel links with a capacity of $100 \mathrm{MB} / \mathrm{sec}$.

- Event assembly by DMA engines on communication controllers to reduce CPU and bus usage.

- Distributed flow control by a window like algorithm. This allows high link utilization without special hardware elements to implement control functions.

- Distributed load balancing by simple algorithms. The full connectivity of the data switch allows flexible load balancing algorithms without changes to the hardware configuration.

- Modular construction to allow incremental performance upgrades. Small versions can be used for detector development and test beam work. 
- Use of a commercially supported ANSI standard protocol. This will reduce engineering costs, provide vendor supported software, and allow cost-effective upgrades.

A prototype test bed has been constructed. This prototype is based on 16 channel Fiber Channel data switch modules and VME based processors and communication controllers. This test bed is being used to determine the performance a Fiber Channel based event builder.

\section{Pablication}

W.H. Greiman, S.C. Loken, and C.P. McParland, "Use of Commercial Gigabit Data Switches for SSC and LHC Event Builders," Proceedings of the CHEP92 Conference on Computing in High Energy Physics, Annecy, France, September 21-25, 1992

\section{Converting Vice to Virtue: Using Crude Time Walk Me isurements to Improve Spatial Resolution in Silicon Tracking Detectors and Simplify Electronic and System Design}

Principal Investigators: David Nygren and Jacques Millaud

Funding: $\$ 74,700$

\section{Project Description}

The central role of silicon detector systems for future high-luminosity physics programs is clear. The complexity of the interesting events will be very great, and the daia rates unprecedented. Pixel vertex detectors could have an enormously beneficial impact on pattern recognition and secondary vertex detection. The pixel vertex detector permits the use of an entirely different, linear track-finding algorithm that may increase event reconstruction speeds by several orders of magnitude using simple specialized processors. The improved secondary vertex detection efficiency has direct impact on $\mathrm{t}$ quark detection through subsequent $b$ quark decays. Pixel detectors will be especially valuable for firture fixed target experiments, which will involve very high instantaneous rates and event complexities. The role of pixel detectors for a B factory may be aiso very important since high-resolu- tion three-dimensional data can be obtained with very low mass. The application to relativistic heavy-ion collisions is especially germane due to the high multiplicity of these events.

To realize a pixel vertex detector suitable for these contexts requires novel and imaginative very large scale integration (VLSI) design concepts to cope with the extreme'y high average rates, storing potentially interesting track data either on-pixel or on-periphery until a level I trigger is available.

This effort began with a new idea, to transfer analog information from the pixel cell to peripheral circuitry using an intrinsic correlation between circuit response and the input signal charge, the so-called time-walk effect. This attractive idea, in addition to removing considerable circuitry from the pixel cell, thereby making it smaller, also has the effect of transforming the system into an essentially digital one, with instantaneous conversion of the analog information to digital form. Several very important advances have germinated from this concept. In fact, the analysis and optimization of pixel vertex detector architecture has proven to be extremely fertile.

\section{Accomplishments}

The original concept of time-walk measurement has been a springboard for several important new ideas. The time-walk concept, by initiating further analysis of the possible role of peripheral circuitry, led to a natural further step, namely of measuring both leading and trailing edges of the comparator response. By appropriate design of the comparator and the signal shaping elements, a nearly linear time-over-threshold relationship has been produced. This holds even for a saturated condition that may occur during very large signals due to Landau fluctuations. The result is an excellent measurement of the deposited charge and hence improved spatial resolution; an improved system diagnostic tool is also automatically achieved.

The second advance, stimulated directly by the timeover-threshold advance, is decentralized signal acquisition. The reevaluation of the peripheral circuitry role, as described in the previous paragraph, led to the realization that the basic recording process of the pixel cell hits can be implemented with locally controlled logic. Here, "local" refers to individual rows and columns, as opposed to the aggregate array of all rows and columns. Previously, it had been held that the basic recording process would be controlled by central logic for the entire array; this has the undesirable consequence that the central logic activity rate becomes excessive very near the design luminosity. 
With decentralized signal acquisition, with each row and column acting locally, the basic activity rates are reduced from $5 \mathrm{MHz}$ to about $78 \mathrm{KHz}$. This change to local control also leads to deadtimeless read/write capability. It also effectively removes constraints on the array size, and allows several readout IC's to be abutted together on one larger detector array.

The third advance, based on the previous two, is the realization is that it appears possible to read the twodimensional pixel address along a single dimension. This column-based architecture decouples $x$ and $y$ activity completely across the array, simplifying certain challenging aspects that the VLSI designer must face. In this new approach, it becomes possible to arrange the sensitive signal amplifier/shaper/ comparator so that no digital lines cross that domain. This offers advantages for noise immunity and robustness as the array size is increased. The penalty sustained in this approach is an increase in pixel cell area in order to incorporate an internal address for the second dimension. The penalty appears to be modest, as the address circuitry can be shared between adjacent columns.

As an outgrowth of these other advances, it has been realized that the time-over-threshold architecture, by providing such good charge resolution, permits a relaxation of the time-walk requirements. This in turn allows some welcome circuit simplification and substantial power dissipation savings.

In conclusion, a concrete, stable, and powerful architectural concept has emerged for the design of smart pixel arrays for operation in high-intensity environments. Analysis of the time-walk effect was a fertile springboard for many new ideas. The major result of all this activity will be the demonstration of pixel vertex detector feasibility. This technology should have wide application throughout high-energy physics and relativistic heavy-ion physics. New collaborators from Italy have joined as a consequence of the advances made possible by this project.

\section{New Si Microstrip Disk Detector for Vertex Reconstruction of B Meson Decays}

Principal Investigators: Alan R. Clark, Frederick R. Goozen, Ronald J. Madaras, Natalie Roe, Mark W. Strovink, Thomas G. Trippe, Stuart A. Kleinfelder, and Roger R. Stone

Funding: $\$ 178,000$

\section{Project Description}

To meet new demands for precise 3-dimensional reconstruction of vertices from decays of $B$ mesons produced at forward angles, we developed a new type of vertex detector, the Silicon Microstrip Disk (SMD). It consists of wedge-shaped double-sided Si microstrip detectors that are joined at the edges to form an open polygon, supported and read out at the outer radius. New techniques to be developed include reading out a Si detector with variable strip length, reading out both sides of a 2-sided detector from the same edge, mounting of the readout electronics directly on the $\mathrm{Si}$ detector, and cooling and support of the disk from the outer radius.

\section{Accomplishments}

Substantial progress has been made toward meeting the goals outlined above for the SMD detector development project. The most significant achievements are summarized below.

Electronic Readout. A conceptual design for the electronic readout of the SMD was developed. The readout elements consist of a custom VLSI chip with very good signal to noise, signal storage and digitization, and sparse readout capability; a high-density interconnect (HDI) on which eight readout chips are mounted; and a low-mass cable that provides power and signal connections to several HDIs. One HDI is 
mounted on each side of a double-sided silicon wedge detector, and one cable can service several detectors.

All three of these items have been designed and prototyped. The readout chip was fabricated in "kit chip" form, each part being fabricated individually in order to study and optimize each feature independently. The HDI was designed and fabricated as a 4 layer flexible kapton circuit with very fine pitch in order to meet the low-mass requirements. SVX readout chips have been wire-bonded to the HDI's, and electrical tests indicate that it functions properly. The HDI layout is shown in Figure 3. The cable design employs Futurebus transceivers to buffer the incoming and outgoing signals; the transceivers have been tested together with the micro-strip cable, and it has been demonstrated that the bandwidth is adequate and the crosstalk is very low. A model SMD showing the connections between the HDIs and the cable has been constructed; the connectors employ a low-mass material called Fuji-poly that makes a good electrical connection when it is squeezed between two conductors.

Cooling. The readout electronics will generate a significant amount of heat which must be removed in order to maintain the silicon detectors at the desired temperature of about $20^{\circ} \mathrm{C}$. Several cooling schemes have been studied and evaluated. Turbulent water cooling was shown to be adequate, and a design employing a loop of aluminum tubing in good thermal contact with the ICs on the disk has been chosen.

Experimental measurements of flow rate vs. pressure drop have been performed, and the experimental friction factor in the cooling loop was found to be $35 \%$ higher than theoretically predicted. This is explained by pipe roughness and by undeveloped flow. The implication is that a greater head than is calculated theoretically will be required for effective cooling. In another cooling experiment, highly turbulent flow in the cooling loop was found to induce less than $2 \mu \mathrm{m}$ of vibration (none was observed). The limit was set by observing the output of a piezoelectric transducer in contact with the tube.

The preliminary conclusion, even with these new doses of reality, is that the temperature difference between the water inlet and the outside of the cooling tube can be held under $5^{\circ} \mathrm{C}$ with a cooling system weight, corrected for radiation length relative to $\mathrm{Si}$, which is $\sim 1 / 3$ that of the active detectors.

Mechanical Support. The mechanical support is divided into two areas: the mechanical support of the 12 wedges that form a disk and the mechanical support of the disk itself that is necessary to precisely align it with respect to the beamline and the other detector elements. The former we call the disk support, and the latter is referred to as the spaceframe.

The design for the disk support uses, the cooling tube as the main structural member at the outside diameter, relying on the stiffness of the wedge detectors themselves to provide the structural connection to a lowmass inner ring. This is a "minimum mass" solution. A premium is placed on being able to construct a cooling ring of high-dimensional stability and tolerance. Experiments have indicated that annealing the cooling ring in a precision fixture is the key to this capability.

Several candidate geometries for the spaceframe were modeled by finite element analysis. Three materials (Al, Be, and graphite-epoxy) were considered, and approximately satisfactory solutions were found for each. The final design which was chosen uses $\mathrm{Al}$ tubing, formed into toroids that are coaxial with the beamline, and struts connecting the toroids. In a conceptual breakthrough, the struts were reoriented

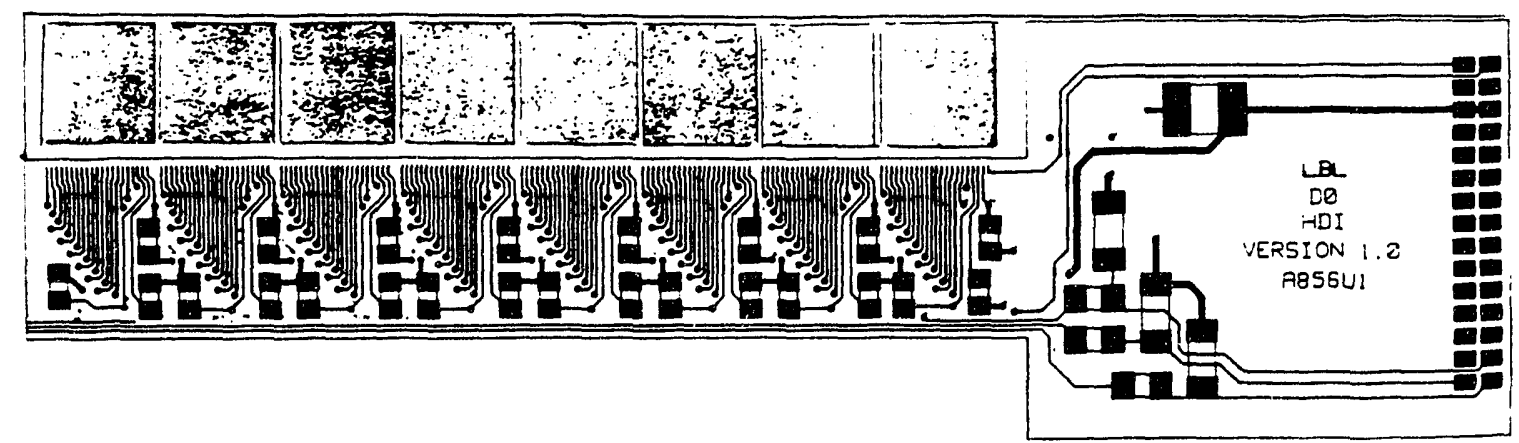

Fig. 3. Layout of the high-density interconnect circuit. The actual size is $8 \mathrm{~cm} \times 1.5 \mathrm{~cm}$. The readout chips are located on the eight pads along one side. 
not to remain parallel with the beamline. Instead, two adjacent struts form a vertex on one toroid and are separated by $1 / 6$ of the azimuth on the adjacent toroid, so that the facets of the spaceframe are triangles rather than rectangles (see Figure 4). This increases the finite-element-simulated stiffness of the frame by one order of magnitude.

The construction technique for the spaceframe is still under study. A model section of the spaceframe was fabricated using soldered joints; it had three times the

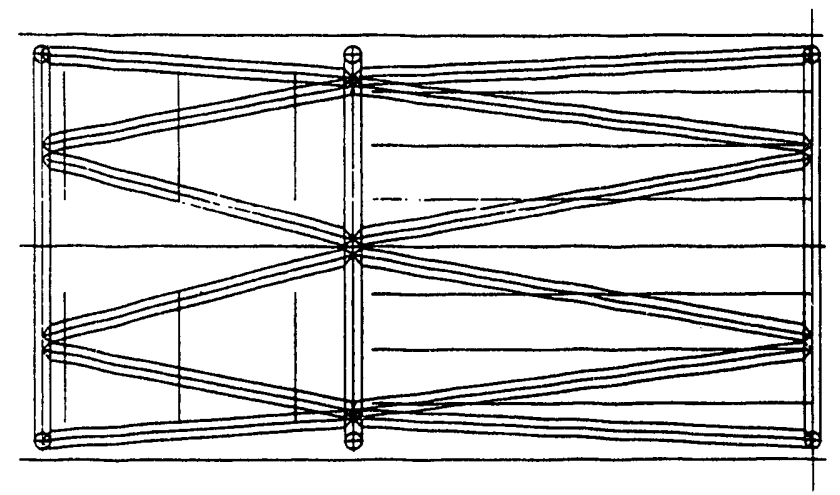

Fig. 4. Side view of the spaceframe design. Disks are attached to the toroidal sections using Tefzel ball joint mounts. calculated stiffness, owing to conservatism in the calculation concerning the degree to which the struts and toroids are simply supported at their joints. However, the thermal expansion of the Al tubing caused by soldering made it difficult to maintain the necessary dimensional tolerances, and it has been decided that the assembly technique should evolve to one in which the joints are epoxied rather than soldered. This technique is now under study and looks promising.

A conceptual design has also been developed for kinematic mounts to connect the disks to the spaceframe. The design is based on the use of Tefzel ball joints, which are located in cylindrical grooves. The design is such that each mount will constrain in two dimensions only so that with 3 kinematic mounts each disk will have 6 constraints, the minimum required. A series of prototype kinematic mounts for attaching the Si detector elements to the spaceframe have been constructed and evaluated.

\section{Publication}

N.A. Roe, "Radiation Damage to Silicon Detectors and Choice of Operating Temperature," D0 Note No. 1380, unpublished. 


\section{Structural Biology Division}

\section{$X$-ray Microscopy and Microimaging of Eukaryotic Chromosomes}

Principal Investigator: John Hearst

Funding: $\$ 48,400$

\section{Project Description}

The purpose of this project is the development of new procedures for the preparation of biological samples of chromatin, chromosomes, and interphase nuclei that will retain the in vivo structural integrity of these samples while at the same time provide greatly enhanced contrast so that the actual organization of DNA in these larger structures can be observed by $x$ ray microscopy. The principal strategies all involve specific delivery of heavy metal atoms to the DNA, so that its presence will be observable in the background of the proteins in these structures.

Many years ago, many observations were made in the electron microscopy of chromatin fibers and metaphase chromosomes. Among the procedures that were used was the staining of the chromatin with a variety of heavy metal ions such as copper, iron, mercury, and tungsten. These preparations showed the beaded structure of polynucleosomes and provided remarkable contrast relative to the surrounding background. While these procedures were not published, because they failed to reveal any new information about nucleosomes, the possibility of the development of $x$-ray microscopy makes these techniques interesting once again. Prior experience with electron microscopy, together with the extensive background with nucleic acid structure and chemistry, provides the basis for this project. Further, DNA supercoiling has recently become an issue of major interest to us. Such issues are of major importance to the ultimate understanding of chromosome structure.

\section{Accomplishments}

We have prepared two samples of terbium chelate, one has terbium chelate bound to silica beads, the other is a precipitate of the chelate itself into fine particles. These samples are being tested at the $x$-ray microscope in Brookhaven for the efficacy of the metal staining procedure for $x$-ray microscopy. The materials are also being tested to establish the kinetics of destruction of the chelate by the high-energy photons.

During a visit to the BESSY Synchrotron Facility in Berlin, we obtained images of E. coli cells, of human sperm, and of human metaphase chromosomes. These images were highly instructive in providing insights into the mechanisms of contrast in the microscope, suggesting immediate steps be taken in mathematical modeling of biological materials such as proteins, nucleic acids, carbohydrates, and lipids for their relative absorptivities as a function of $x$-ray wavelength in the spectral region that is anticipated to be available. In particular, there are essential issues of the availability of both sides of the oxygen edge, and perhaps other absorption edges as well.

The observations on the unfixed human metaphase chromosome spread were quite surprising and provide considerable optimism relating to the potential utility of the Advanced Light Source microscope in establishing the coiling structure of metaphase chromosomes. The $x$-ray microscope would provide a tool for studying the forces involved in chromosome condensation, the mechanical properties of the largest chromosome fibers, the physical arrangement of such fibers during the reversal of the condensation process, and ultimately a mapping tool for the genetic markers on structural domains within the chromosome. We observed the rapid expansion of metaphase chromosomes upon exposure to $x$-rays, suggesting that they may be condensed by torsional stress in the structural fibers that is released by the $x$-ray exposure. Such release results in a rapid change in structure in air. Following this rapid change, the structures are stable to further $x$-ray irradiation, at least by $x$-ray imaging, for a period involving at least ten times the exposure initiating the initial response. What is clear is that time resolved observations are possible, adding a dimension to microscopy at this resolution that can clearly not be provided by electron microscopy. 


\section{Novel X-Ray Crystallographic Studies of $R N A$}

Principal Investigator: Stephen R. Holbrook

Funding: $\$ 68,100$

\section{Project Description}

Despite playing a central role in many cellular processes, little is known about the complex and varied structures that ribonucleic acid (RNA) may assume in order to carry out its biological functions. Although considerable effort has been made toward determination of RNA structure, to date only a few RNA structures have been deterinined. These have provided a glimpse of the complexity that these molecules may assume. However, complex RNA structural elements such as pseudoknots, hairpin loops, parallel and multistranded helices, and unusual tertiary base pairing schemes have yet to be explored by $x$-ray crystallography. Now that methods of RNA synthesis are at hand whereby large quantities of highly purified and homogeneous material can be obtained, advances in the methods of crystallization and crystal structure determination are needed in order to further this field.

This project focuses on developing crystallization and structure solution techniques for application to RNA structure determination by $x$-ray crystallography. Studies in FY92 have concentrated on development of crystallization methods and obtaining suitable crystals for which structure solution methods can be developed in the second year of support. These techniques will be tested by application to structural studies of: (1) a family of RNA sequences that form highly stable hairpin loops; (2) sequences forming mismatched basepairs or internal loops; (3) RNA oligomers that can form pseudoknot structures; and (4) RNA elements that serve as protein recognition sites, i.e. the TAR RNA and RRE RNA, which serve as recognition sites of the HIV-1 Tat and Rev proteins.

\section{Accomplishments}

We have made excellent progress in the development of crystallization techniques, growth of crystals, and in the initial stages of crystal, structure analysis of several RNA oligonucleotides. Our progress is detailed below.

Development of Crystallization Techniques. Oligonucleotide Design: We are taking a comparative approach to the design of crystallizable oligonucleotides. In this approach we are evaluating the effects of oligomer length and the presence of a 5'-phosphate and of small sequence variations on whether the oligomers will form crystals. Specifically, crystallization was compared for (1) a series of RNA and DNA oligomers containing consecutive G-A, A-G mismatches, and a dodecamer containing the same mismatches. For the RNA octamer sequences $\mathrm{r}-$ GGCGAGCC and $\mathrm{r}-$ GICGAGCC, it was possible to grow large single crystals suitable for diffraction studies, while the DNA analogues d-GGCGAGCC and d-CGCGAGCG formed only poor crystals and no crystals, respectively. The RNA dodecanucleotide r-GGAGCGAGCUCC did not crystallize at all. (2) An RNA dodecamer including the tetraloop forming sequence GAAA either with a 5'terminal monophosphate group or no phosphate at all. This oligomer formed good crystals when no 5'terminal phosphate was present and no crystals with a 5 -terminal monophosphate. Finally, (3) two dodecamer RNAs in which the central four bases are either UUCG or UUUG and the rest of the sequence is identical were crystallized. Although crystals were obtained for both sequences, those from UUCG were far superior. These experiments are still in a preliminary stage and must be approached carefully so as to identify differences due to intermolecular interactions that may be important for crystallization.

Construction of a Nucleic Acid Crystallization Screening Matrix. We have designed a set of 24 trial crystallization conditions for initial screening of oligonucleotides. The screen is analogous to the protein crystallization screen of Jancarik and Kim, but has been designed to include conditions previously shown to induce RNA or DNA crystallization. In addition, this screen generally uses low ionic strength conditions, both because these are suitable for highly polar oligonucleotides and to complement the high ionic strength conditions of the protein screen designed by Jancarik. So far, this screen has been successful in providing initial crystals of several RNAs including the GAAA oligomer described below. Recently, we have designed a second nucleic acid screening matrix and are in the process of testing it in crystallization of several RNA oligonucleotides. These two screening matrices are shown in Figure 1.

Crystallization, Space Group Determination and X-Ray Data Collection of RNA Oligomers. We have succeeded in obtaining diffraction quality crystals of (1) a sequence (GAAA tetraloop) that can form either a single stranded stem-loop structure or a double stranded structure containing an internal loop of four consecutive mismatched base-pairs and (2) several different sequences that can form duplexes containing internal loops of two consecutive G-A (A-G) mismatched basepairs. Data collected from these crystals will be used 


\begin{tabular}{|c|c|c|c|c|c|}
\hline No. & $\begin{array}{c}\text { Monovalent } \\
\text { Cation }\end{array}$ & $\begin{array}{c}\text { Divalent } \\
\text { Cation }\end{array}$ & Polyamine & Buffer & Precipitant \\
\hline \multicolumn{6}{|c|}{ Screen 1} \\
\hline $\begin{array}{l}1 . \\
2 . \\
3 . \\
4 . \\
5 . \\
6 . \\
7 . \\
8 . \\
9 . \\
10 . \\
11 . \\
12 . \\
13 . \\
14 . \\
15 . \\
16 . \\
17 . \\
18 . \\
19 . \\
20 . \\
21 . \\
22 . \\
23 . \\
24 .\end{array}$ & $\begin{array}{l}\overline{\mathrm{Na}} \\
\overline{\mathrm{K}} \\
\mathrm{K} \\
\mathrm{NH4} \\
\mathrm{Na} \\
\mathrm{Na} \\
\overline{\mathrm{Na}} \\
\overline{\mathrm{NH}} \\
\mathrm{Na} \\
\mathrm{Na} \\
\mathrm{K} \\
\mathrm{NH4} \\
\mathrm{Na} \\
\overline{\mathrm{Na}} \\
\mathrm{K} \\
\mathrm{Na} \\
\mathrm{K} \\
\overline{\mathrm{Na}} \\
-\end{array}$ & $\begin{array}{l}\mathrm{Mg} \\
\mathrm{Mg} \\
\overline{\mathrm{Mn}} \\
\mathrm{Ba} \\
\mathrm{Cd} \\
\mathrm{Zn} \\
\overline{\mathrm{Mg}} \\
\mathrm{Cd} \\
\overline{\mathrm{Mg}} \\
\mathrm{Mn} \\
\mathrm{Cd} \\
\mathrm{Mg} \\
\mathrm{Mg} \\
\mathrm{Mg} \\
\overline{\mathrm{Mg}} \\
\overline{\mathrm{Mg}} \\
\mathrm{Ba} \\
\overline{\mathrm{Mg}}\end{array}$ & $\begin{array}{l}\text { Sper. } \\
\text { Sper. } \\
- \\
- \\
- \\
- \\
\text { Sper. } \\
\frac{\text { Sper. }}{-} \\
- \\
\overline{-} \\
\text { Sper. } \\
- \\
- \\
- \\
- \\
\text { Sper. }\end{array}$ & $\begin{array}{l}\text { Cacodylate } \\
\text { Hepes } \\
\text { Tris } \\
\text { Hepes } \\
\text { Cacodylate } \\
\text { Cacodylate } \\
\text { Hepes } \\
\text { Cacodylate } \\
\text { - } \\
\text { Tris } \\
\text { Hepes } \\
\text { Cacodylate } \\
\text { Tris } \\
\text { Cacodylate } \\
\text { Hepes } \\
\text { Cacodylate } \\
\text { Cacodylate } \\
\text { Tris } \\
\text { Hepes } \\
\text { Cacodylate } \\
\text { Cacodylate } \\
\text { Hepes } \\
\text { Hepes } \\
\text { - }\end{array}$ & $\begin{array}{l}35 \% \text { MPD } \\
35 \% \text { MPD } \\
30 \% \text { MPD } \\
35 \% \text { MPD } \\
30 \% \text { MPD } \\
35 \% \text { MPD } \\
30 \% \text { MPD } \\
15 \% \text { Isopropanol } \\
15 \% \text { Isopropanol } \\
15 \% \text { Isopropanol } \\
30 \% \text { PEG } 400 \\
35 \% \text { PEG } 400 \\
35 \% \text { PEG } 400 \\
35 \% \text { PEG } 400 \\
35 \% \text { PEG } 600 \\
30 \% \text { PEG } 1000 \\
35 \% \text { PEG } 4000 \\
35 \% \text { PEG } 8000 \\
1.2 \text { M Amm. Sulfate } \\
1.2 \text { M Amm. Sulfate } \\
1.2 \text { M Sodium Acetate } \\
1.2 \text { M Sodium Acetate } \\
40 \% \text { t-Butanol } \\
15 \% \text { Acetone }\end{array}$ \\
\hline \multicolumn{6}{|c|}{ Screen 2} \\
\hline $\begin{array}{l}25 . \\
26 . \\
27 . \\
28 . \\
29 . \\
30 . \\
31 . \\
32 . \\
33 . \\
34 . \\
35 . \\
36 . \\
37 . \\
38 . \\
39 . \\
40 . \\
41 . \\
42 . \\
43 . \\
44 . \\
45 . \\
46 . \\
47 . \\
48 .\end{array}$ & $\begin{array}{l}\mathrm{Li} \\
\text { Arg. } \\
\mathrm{Na} \\
\overline{\text { Arg }} \\
\mathrm{NH} 4 \\
\mathrm{~K} \\
\overline{\mathrm{NH}} 4 \\
\mathrm{Arg} \\
\mathrm{K} \\
\overline{\mathrm{Na}} \\
\overline{\mathrm{Li}} \\
\overline{\mathrm{Arg}} \\
\mathrm{Ba} \\
\mathrm{Na} \\
\overline{-} \\
\overline{\mathrm{Li}} \\
\mathrm{NH} 4 \\
\overline{\mathrm{K}}\end{array}$ & $\begin{array}{l}\mathrm{Mg} \\
\mathrm{Mn} \\
\mathrm{Zn} \\
\overline{\mathrm{Co}} \\
\overline{\mathrm{Mg}} \\
\mathrm{Ba} \\
\overline{\mathrm{Ba}} \\
\mathrm{Ca} \\
\mathrm{Co}(\text { hex) } \\
\overline{\mathrm{Co}} \\
\mathrm{Co}(\text { hex) } \\
\mathrm{Cd} \\
\mathrm{Sm}+3 \\
\overline{\mathrm{Zn}} \\
\mathrm{Ca} \\
\mathrm{Co} \\
\overline{\mathrm{Cd}} \\
\mathrm{Ba}\end{array}$ & $\begin{array}{l}\overline{\mathrm{Sm}^{+3}} \\
\overline{\mathrm{Ru}^{+3}} \\
\overline{\text { Putrs }} \\
\overline{-} \\
\mathrm{Sm}^{+3} \\
\text { Sperd } \\
\overline{-} \\
\mathrm{Ru}^{+3} \\
\overline{\text { Putrs }} \\
\overline{\text { Cacodylate* }} \\
\text { Ru+3 } \\
\text { Sperd } \\
\overline{-} \\
\text { Sperm } \\
\end{array}$ & $\begin{array}{l}\text { Cacodylate } \\
\text { Cacodylate* } \\
\text { Tris* } \\
\text { Tris } \\
\text { Hepes } \\
\text { Cacodylate* } \\
\text { Tris* } \\
\text { Cacodylate* } \\
\text { Tris* } \\
\text { Cacodylate* } \\
\text { Cacodylate } \\
\text { Hepes } \\
\text { Tris* } \\
\text { Cacodylate } \\
\text { Cacodylate* } \\
\text { Cacod } \\
\text { Tris* } \\
\text { Hepes } \\
\text { Cacodylate } \\
\text { Cacodylate* } \\
\text { Tris } \\
\text { Hepes } \\
\text { Tris }\end{array}$ & $\begin{array}{l}35 \% \text { MPD } \\
30 \% \text { MPD } \\
30 \% \text { MPD } \\
30 \% \text { MPD } \\
30 \% \text { MPD } / 10 \% \text { PEG } 1000 \\
30 \% \text { MPD } / 10 \% \text { PEG } 400 \\
30 \% \text { MPD } / 5 \% \text { Isopropanol } \\
20 \% \text { Ethanol } \\
20 \% \text { Ethanol } \\
30 \% \text { PEG } 400 \\
30 \% \text { PEG } 400 \\
35 \% \text { PEG } 400 \\
35 \% \text { PEG } 400 \\
35 \% \text { PEG } 600 \\
35 \% \text { PEG } 600 \\
30 \% \text { PEG } 1000 \\
35 \% \text { PEG } 4000 \\
35 \% \text { PEG } 8000 \\
35 \% \text { PEG } 8000 \\
20 \% \text { Dioxane } \\
20 \% \text { Dioxane } \\
20 \% \text { Dioxane } \\
40 \% \text { t-Butanol } \\
15 \% \text { Acetone }\end{array}$ \\
\hline \multicolumn{6}{|c|}{ 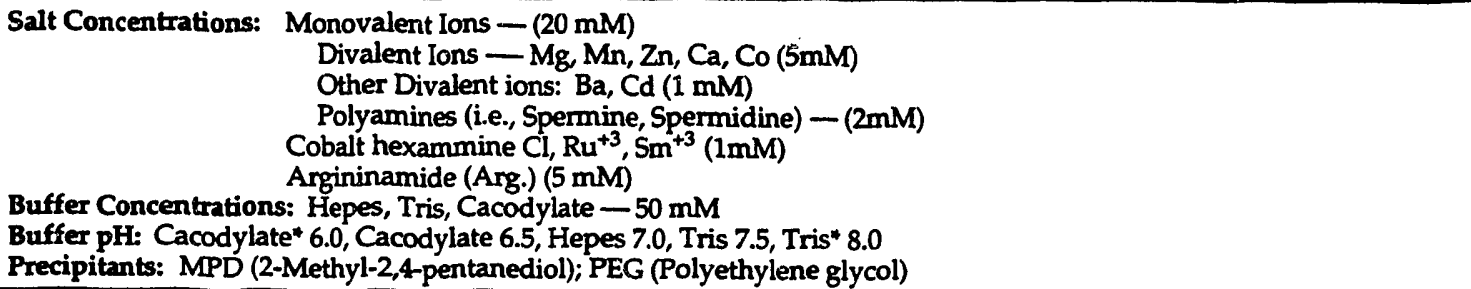 } \\
\hline
\end{tabular}

Fig. 1. Nucleic acid crystallization screens. 
to develop methods of crystal structure solution for RNA.

GAAA Tetraloop Sequence: The RNA dodecamer rGGCCGAAAGGCC was crystallized from the nucleic acid screen described above. The largest crystals measure $0.35 \times 0.2 \times 0.2 \mathrm{~mm}$. We have used precession photography to establish the space group of this RNA dodecamer as $\mathrm{P}_{1} 22$ (or $\mathrm{P} 6_{5} 22$ ); $\mathrm{a}=\mathrm{b}=37.7 \AA \mathrm{c}=88.3$; $\alpha=\beta=90^{\circ} \gamma=120^{\circ}$. The volume calculated per asymmetric unit is consistent with the presence of either a single stranded hairpin or a dodecanucleotide double helix utilizing a crystallographic two-fold axis of symmetry. Diffraction data have been collected to $2.5 \AA$ resolution.

G-A Mismatches: Consecutive G-A, A-G mismatches can be formed by the octanucleotide duplex ( $r$ GGCGAGCC) 2. We have obtained crystals of maximal size $0.80 \times 0.20 \times 0.20 \mathrm{~mm}$ by ammonium sulfate precipitation in the presence of PEG 400. Diffraction data have been collected in the hexagonal space group
$\mathrm{P}_{2}$ to a resolution of $2.5 \AA$ using an area detector system with a phosphor image plate detector system. We have also collected data on isomorphous crystals of the RNA octamer (r-GICGAGCC) where inosine replaces guanine in the second position. Also, a DNA analogue of this RNA sequence has been crystallized (d-GGCGAGCC), and the space group determined to be I222. PEG 400 was also used as the precipitant for crystallization of this DNA, but in this case $\mathrm{Zn}^{+2}$ was found to be a necessary counterion.

It is expected that a switch in the order of $G$ and $A$ in the mismatch region of the sequence will make a large difference in the structure. With this in mind we have crystallized oligomers containing consecutive $A-G, G-$ A mismatches as opposed to the G-A, A-G mispairs discussed above. The RNA sequences ( $r$ CGCAGGCG) and ( $r-C G C A I G C G)$ as well as ( $r-$ GGCAGGCC) have been crystallized, but the crystals are not yet of sufficient quality to collect diffraction data. 


\section{Acronyms and Abbreviations}

\begin{tabular}{|c|c|}
\hline AFM & atomic force microscopy \\
\hline ALS & Advanced Light Source \\
\hline $\mathrm{CCD}$ & charge-coupled device \\
\hline $\mathrm{DE}$ & discrete event \\
\hline DLTS & deep level transient spectroscopy \\
\hline DOE & U.S. Department of Energy \\
\hline DSDP & Deep Sea Drilling Project \\
\hline DUV & deep ultraviolet \\
\hline EBSP & electron back scattering patterns \\
\hline ECR & electron cyclotron resonance \\
\hline EM & electromagnetic \\
\hline EMCS & energy management control systems \\
\hline EPR & electron paramagnetic resonance \\
\hline EPRI & Electric Power Research Institute \\
\hline EREM & extended resolution electron microscopy \\
\hline ESD & energy dispersive spectrometry \\
\hline EXAFS & extended $x$-ray absorption fine structure spectroscopy \\
\hline FEL & free electron laser \\
\hline GIS & Geographic Information System \\
\hline HDI & high-density interconnect \\
\hline HDL & high-density lipoprotein \\
\hline HMEC & human mammary epithelial cell \\
\hline ISL & IsoSpin Laboratory \\
\hline LANL & Los Alamos National Laboratory \\
\hline LBL & Lawrence Berkeley Laboratory \\
\hline LCAT & lecithin:cholesterol acyltransferase \\
\hline LDL & low-density lipoprotein \\
\hline LDRD & Laboratory Directed Research Development (Program) \\
\hline LGM & Last Glacial Maximum \\
\hline MBE & molecular beam epitaxy \\
\hline MMM & Magnetic Materials Microscope \\
\hline MOCVD & Metal-Organic Chemical Vapor Deposition \\
\hline NCEM & National Center for Electron Microscopy \\
\hline NMR & nuclear magnetic resonance \\
\hline
\end{tabular}




\begin{tabular}{ll} 
NRE & Non-Recurring Engineering \\
NSAC & Nuclear Science Advisory Committee \\
ODMR & optical/luminescence detected magnetic resonance \\
ODP & Ocean Drilling Program \\
PAM & pulse amplitude modulated \\
PG\&E & Pacific Gas \& Electric Company \\
PDE & particle induced x-ray emission \\
PL & photoluminescence \\
PTLP & particle transient liquid phase \\
QSAM & Quantitative Sub-Angstrom Microscope \\
QTL & Quantitative Trail Loci \\
R\&D & research and development \\
SEM & scanning electron microscopy \\
SIS & superconductor-insulator-superconductor \\
SLAC & Stanford Linear Accelerator Center \\
SMD & Silicon Microstrip Disk \\
SQUID & Superconducting QUantum Interference Devices \\
SSR & simple sequence repeats \\
SSRL & Stanford Synchrotron Radiation Laboratory \\
SVT & vicuum ultraviolet \\
TEM & silicon vertex tracking \\
TGM & transmission electron microscopy \\
TLP & Toroidal Grating Monochromator \\
TPC & transient liquid phase \\
UCB & Time Projection Chamber \\
UCLA & University of California at Berkeley \\
UV & University of California at Los Angeles \\
VLSI & ultraviolet \\
VUV & very large scale integration \\
XMCD & vachroism \\
\hline
\end{tabular}



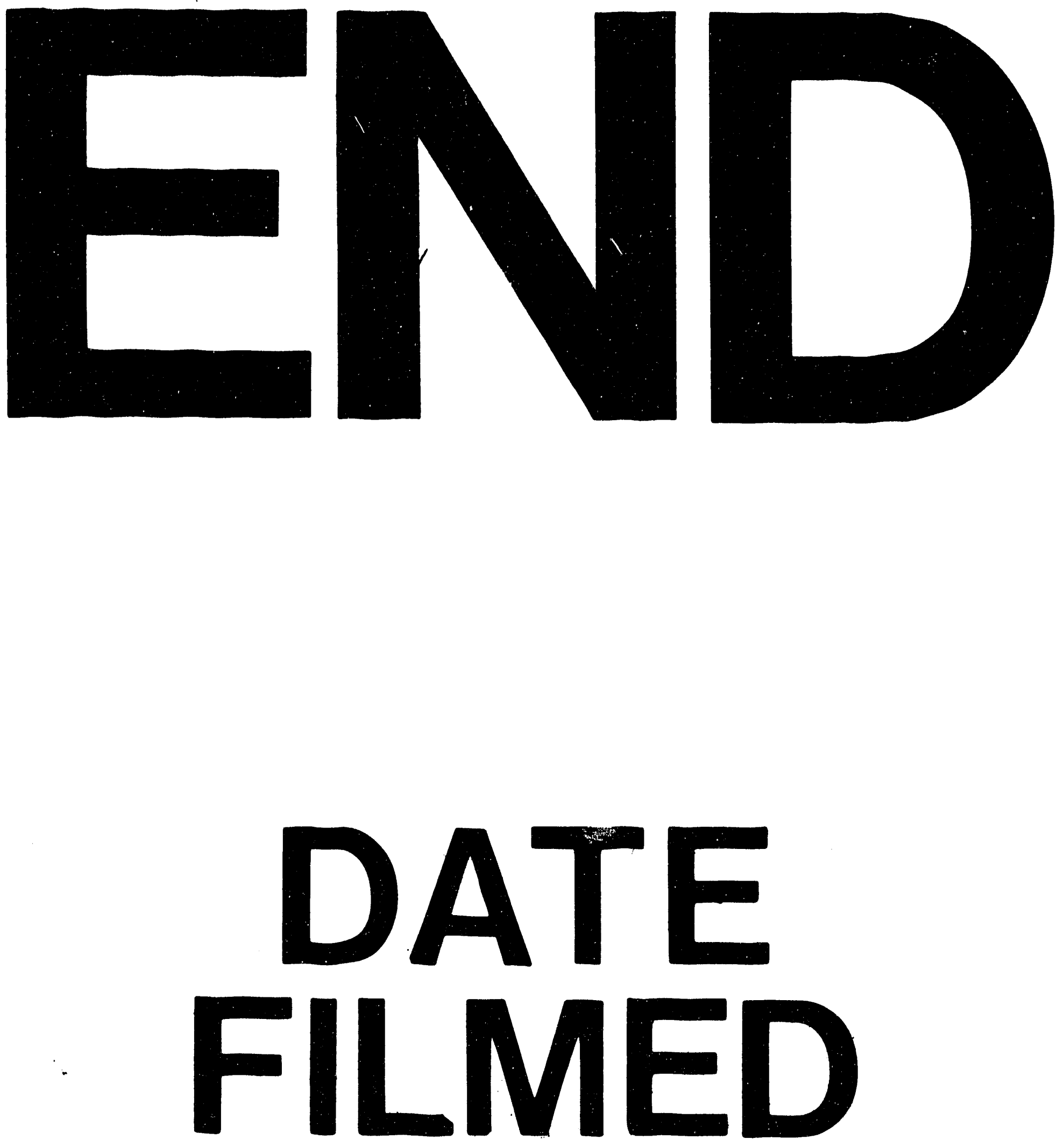

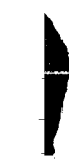

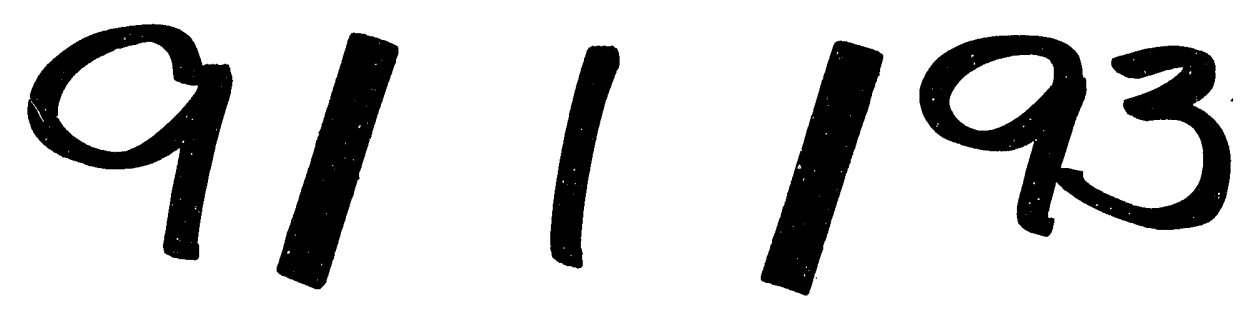


: $\quad$ 